\title{
CROSSED-BEAM CHEMICAL REACTION DYNAMICS \\ PROBED WITH UNIVERSAL AND STATE RESOLVED \\ ION IMAGING
}

\author{
A Dissertation \\ Presented to
}

the Faculty of the Graduate School

at the University of Missouri-Columbia

In Partial Fulfillment

of the Requirements for the Degree

DOCTOR OF PHILOSOPHY

By

ALEXANDER KAMASAH

Dr. Arthur G. Suits, Thesis Supervisor

JULY 2018 
CCOPYRIGHT BY

\section{ALEXANDER KAMASAH}

2018

All Rights Reserved 
The undersigned, appointed by the Associate Vice Chancellor of the Office of Research and Graduate Studies, have examined the dissertation entitled

\section{CROSSED-BEAM CHEMICAL REACTION DYNAMICS PROBED WITH UNIVERSAL AND STATE RESOLVED ION IMAGING}

presented by Alexander Kamasah, a candidate for the degree of doctor of philosophy, Chemistry and hereby certify that, in their opinion, it is worthy of acceptance.

Professor Arthur G. Suits

Professor Timothy Glass

Professor Michael Greenlief

Professor Aigen Li 


\section{DEDICATION}

This dissertation is dedicated to My Wife: Kamasah, Marian; My Kids: Mich and Josh; My parents of blessed memory: 


\section{ACKNOWLEDGEMENTS}

There are quiet a number of individuals that have earned my gratitude for their contribution to my time in graduate school. I would like to first begin by giving thanks to

God for His grace, love and favor during my time in graduate school. Secondly, 1 am very much indebted to my advisor, Dr. Arthur Suits for his patient, encouragement and giving me an opportunity to be part of his wonderful research team. Under his supervision, 1 acquired the needed skills and techniques in my area of research. On a personal level, Dr. Suits always inspired me by his passionate attitude towards work and his love for the scientific community. Thank you very much Boss and it has been a great honor to be mentored by you. I would also like to thank the rest of my dissertation committee members (Dr. Timothy Glass, Dr. Michael Greenlief, and Dr. Aigen Li) for their great support and invaluable advice.

I have been very fortunate to be part of the Suits group and more importantly working on the crossed-beam machine. The experiment were very exciting and challenging but 1 was very fortunate to have worked with a former postdoc, Dr Joalland Baptiste who introduced me to the basics of the cross beam machine. I also learnt a lot from a former senior graduate student Yuauyuan Shi and it was great working with her. I also want to express my appreciation to Dr. Hongwei $\mathrm{Li}$, currently a postdoc in our lab who has been working with me on the crossed-beam machine. He has really been an inspiration and 1 have enjoyed every moment that 1 have worked with him. Thanks to all, past and present Suits members for making my stay in the Suits lab to be very exciting and memorable. I also want to express my appreciation to Dr. Bernadette Broderick for her words of encouragement and support. I am also very grateful to the chemistry department for 
granting me all the needed help and support especially, Jerry Brightwell and Holly Oswald. I also want to express my appreciation to Rod Schlotzhauern and Cheston Callais at the physics department for always be handy to do machining for the crossedbeam machine.

I also want to express my deepest gratitude to my family especially my virtuous wife Marian for her continuous support, understanding and sacrifices. 


\section{PREFACE}

This dissertation is based solely on the following refereed publications:

Chapter 3: Kamasah, A, Li Hongwei, Onvlee, J. Avoird, Ad van der. Parker D. Suits, A,G. Imaging the inelastic scattering of vibrationally excited NO $(v=1)$. Chem. Phys. Letters 692 (2018) $124-128$.

Chapter 4: Hongwei Li, Alexander Kamasah, and Arthur G. Suits. Intersystem crossing in the exit channel. Manuscript under revision. Nature. Chemistry.

Chapter 5: Hongwei Li, Alexander Kamasah, and Arthur G. Suits. Imaging the dynamics of $\mathrm{H}$-Abstraction of $\mathrm{O}(3 \mathrm{P})$ with 1-propanol and 2-propanol. (Manuscript in preparation) 


\section{TABLE OF CONTENTS}

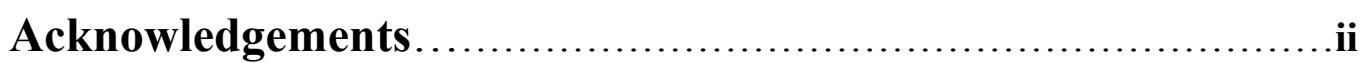

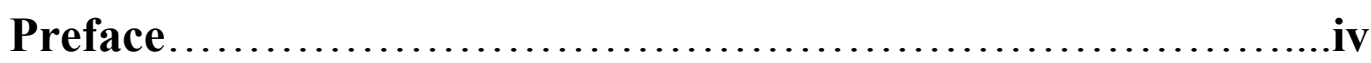

List Tables...........................................................

List of Figures...................................................

Chapter 1-Introduction.......................................1

Chapter 2-Experimental Setup.............................8

2.1 Crossed Molecular Beam............................................. 8

2.2 Universal Detection........................................... 10

2.3 Resonance Enhanced Multiphoton Ionization.......................12

2.4 Ion Imaging ...................................................

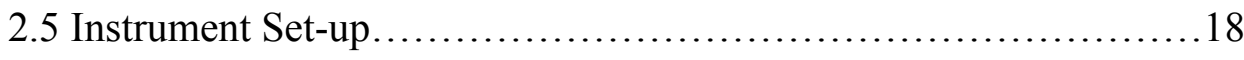

2.6 Piezo-Stack Valve.................................................21

Chapter 3-Imaging the inelastic scattering of vibrationally excited NO ( $v=1)$ with Ar.................................................24

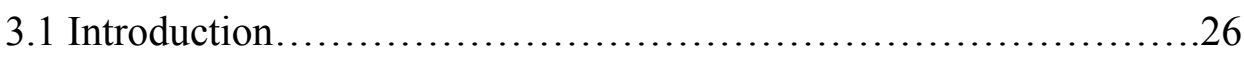

3.2 Experimental Set-up..........................................30

3.3 Results and Discussion......................................... 32

3.4 Conclusion..................................................... 44

Chapter 4-Intersystem crossing in the exit channel..............45

4.1 Introduction.........................................47

4.2 Experimental..........................................48 
4.3 Results and Discussion.................................53

4.4 Conclusion...........................................65

Chapter 5- Imaging $\mathrm{H}$ abstraction dynamics in crossed molecular beams: $\mathbf{O}\left({ }^{3} \mathrm{P}\right)+$ propanol isomers ..................................66

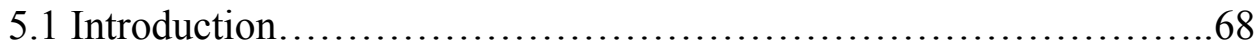

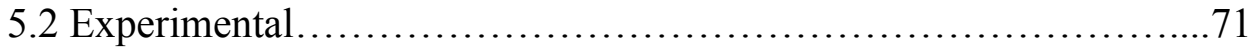

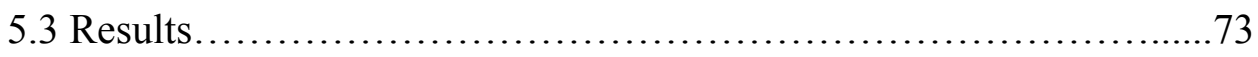

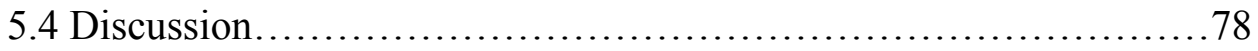

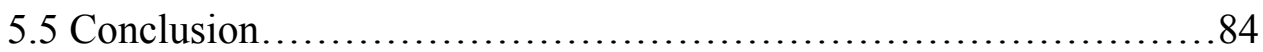

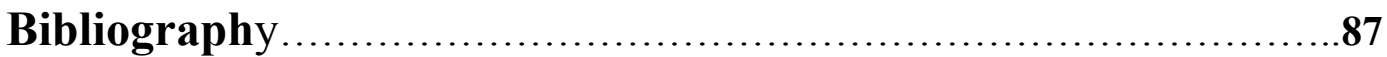

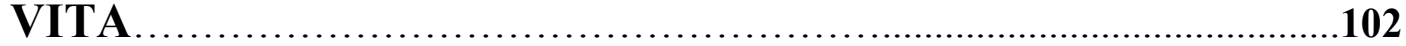




\section{LIST OF TABLES}

TABLE 1.0 Summary of results on the translational energy partitioning

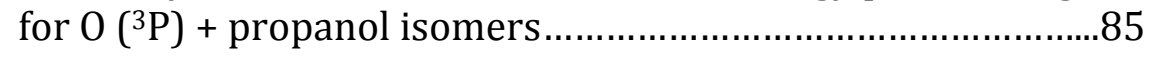

TABLE 2.0 Results of $a b$ initio calculations performed as described in the text (hartrees, degrees, angstroms)............................ 86 


\section{LIST OF FIGURES}

Figure 1.0 Center-of-mass distribution of the $\mathrm{C}_{4} \mathrm{H}_{7}$ radical from the reaction between $\mathrm{C}_{4} \mathrm{H}_{8}$ and $\mathrm{HCl}$.

Figure 1.1 Newton diagram showing the relation between the laboratory and

Center of mass velocities 6

Figure 2.0 Schematic illustration of supersonic expansion of a gas through a nozzle 10

Figure 2.1 Schemes for ionization by resonance-enhanced multiphoton Ionization.

Figure 2.2 Set-up diagram of a conventional photofragment ion imaging apparatus.

Figure 2.3 Schematic views of the crossed-beam apparatus combined with universal sliced dc ion imaging.................................20

Figure 2.4 Schematic view of the valve assembly made of piezostack actuator....22

Figure 3.1 Energy level diagram of NO showing the energy splitting.....

Figure 3.2 $1+1$ REMPI spectra of NO recorded for $\mathrm{v}=0$ (top panel) using $(0,0)$

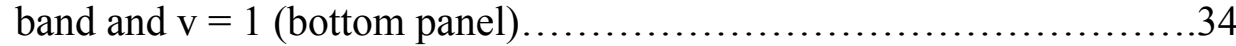

Figure 3.3 DC slice images for inelastic scattering of NO with Ar...............37

Figure 3.4 A comparison of the experimental and theoretical differential cross sections for the scattering of $\mathrm{NO}(\mathrm{v}=0)$ with Ar.

Figure 3.5 Comparisons of the experimental differential cross section distributions for the inelastic scattering of $\mathrm{NO}(\mathrm{v}=0) / \mathrm{Ar}$ (black curve) and $\mathrm{NO}(\mathrm{v}=$ 1)/Ar (red curve)....

Figure 4.1 DC slice images top Dimethylamine and bottom Trimethylamine with Newton diagrams superimposed

Figure 4.2 Center-of-mass angular $\mathrm{T}(\theta)$ (top) and translational energy $\mathrm{P}\left(\mathrm{E}_{\mathrm{T}}\right)$ (bottom) distributions for the reactions of $\mathrm{O}\left({ }^{3} \mathrm{P}\right)$ with TMA (left) and DMA (right).

Figure 4.3 Key points on the triplet (red) and singlet (black) PESs of the $\mathrm{O}\left({ }^{3} \mathrm{P}\right)+\mathrm{TMA}$ reaction. 
Figure 4.4 Key points on the triplet (red) and singlet (black) PESs of the $\mathrm{O}\left({ }^{3} \mathrm{P}\right)+$ DMA reaction........................................60

Figure 4.5 Geometric structure calculated at the wB97XD/6-311G(2d,d,p)

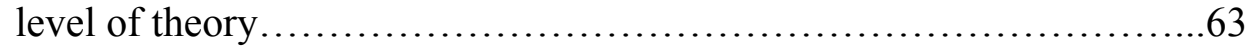

Figure 5.1 Lowest energy structures of 1-propano and 2-propanol calculated at CBS-QB3 level of theory ..................................................................73

Figure 5.2 Sliced scattering images for the reaction of $\mathrm{O}\left({ }^{3} \mathrm{P}\right)$ with 1-propanol (Newton diagrams superimposed on them) .........................75

Figure 5.3 Sliced scattering images for the reaction of $\mathrm{O}\left({ }^{3} \mathrm{P}\right)$ with 2-propanol

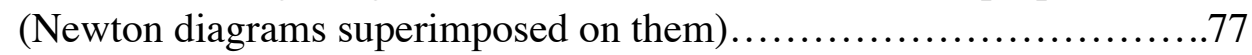

Figure 5.4 Reduced translational energy distributions of the hydroxyalkyl products for low collision and high collision energy....................8 80 


\section{CHAPTER 1}

\section{INTRODUCTION}

The main goal of chemical reaction dynamics is to unravel the intimate motions of individual atoms during a chemical transformation. This information must generally be inferred from indirect macroscopic measurement. Very important information such as translational energy dependence of the reaction cross-section, vibrational mode-specific promotion of reactivity, product angular and velocity distributions are normally extracted. Understanding how these chemical reactions occur at the microscopic level gives us a better insight in understanding reactive intermediates and products of reaction. For a better understanding of the elementary chemical reactions, it is imperative that the studies are performed under well-defined laboratory conditions. Over the last few decades, the field has witnessed unprecedented advances in both experiment and theory.

Advancements in generating reactants, state selection, improvement of crossed-molecular beam machines and products detection have gone a long way to improve our ability in studying chemical reactions in the gas phase. In 1986, Hershbach, ${ }^{1}$ Lee ${ }^{2}$, and Polayni ${ }^{3}$ together shared the Nobel Prize in Chemistry for their work on the dynamics of gas phase reactions.

The study of bimolecular reactions using crossed-molecular beams continues to play pivotal role in understanding reaction dynamics. The single collision conditions of crossed molecular beam experiments permits the unambiguous identification of primary products which is very important in cases where very highly reactive species such as radicals are used as reactants or are formed as products. The experiment is performed by 
crossing two collimated beams (a target beam and radical beam) normally at an angle of $90^{\circ}$, and the resulting products are scattered into different angles. The angle-resolved flux is known as the differential cross section (DCS) ${ }^{4}$ and is probed using different detection schemes such as resonance enhance multiphoton ionizatiom (REMPI) ${ }^{5}$, laser induced fluorescence $(\mathrm{LIF})^{6}$, or DC slice imaging ${ }^{7}$.

This technique provides us with the product velocity distribution, which tells us how much available energy has been partitioned into translational, rotation or internal excitation of the products. The product angular distribution, $I(\theta)$, in the center-of-mass frame gives us the details of the mechanism of the reactions and usually contains some information on the lifetime of the collision complex.

A crossed molecular beam experiment involves bimolecular collisions and these collisions result in scattering processes. Although in crossed-molecular beam experiments we do not have any way of controlling the impact parameter, we can control the relative velocities of the reactant species at specific energies. The same basic law that governs macroscopic collisions, for example, the laws of conservation of momentum and energy, usually governs molecular collisions. In most of these individual collisions, they are characterized by an impact parameter, $b$, the relative velocities of the colliding species $\mathrm{V}_{\text {rel }}$, and the scattering angle $\theta$. Scattering experiments are performed to obtain information on the forces between the colliding particles; In addition, measuring the cross section gives information on the part of interaction potential that is responsible for the energy transfer. Molecular collisions that result in scattering can be categorized into three main types: Elastic, inelastic, and reactive scattering. Elastic scattering is scattering in which translational energy is conserved. The collision partners have the same total 
translational kinetic energy after the collision as they did before the collision and no energy is transferred into internal degrees of freedom. Inelastic scattering is scattering in which kinetic energy is not conserved, and energy is converted between different forms e.g. translational to rotational or vibrational. Inelastic scattering data provide direct information on both the attractive as well as repulsive parts of the intermolecular potentials. Rotational energy transfer is one of the simplest and important scattering processes in collision dynamics and can be described by

$$
\mathrm{A}+\mathrm{BC}\left(J_{i}\right) \rightarrow \mathrm{A}+\mathrm{BC}\left(J_{f}\right)+\Delta \mathrm{E}
$$

Where $\mathrm{A}$ is an atom, $\mathrm{BC}$ is a diatomic molecule, $\mathrm{J}$ is the rotational quantum number of the molecule, $i$ and $f$ are the initial and final states respectively, and $\Delta \mathrm{E}$ is the change in translational energy.

Reactive scattering is a collision process that leads to a chemical change i.e. a collision in which chemical bonds are made or broken so that the species leaving the collision region are chemically distinct from those that entered.

When two molecules collide, the products are scattered in a variety of directions relative to the direction of approach. The scattered products can be described either in the laboratory or center of mass frame, and they are described in terms of scattering angle and the beam velocities. Although, the description of events in the laboratory frame of reference is always the first thing experimentalist look out for, it is more appropriate to describe the collisions in the center of mass frame. For example, in the laboratory frame it is difficult in comparing experimental results obtained for different parameters such as collision angle between two molecular beams. The use of center of mass frame under such scenarios gives experimentalist a better understanding of the collision dynamics. In 
the center of mass frame collisions are characterized in terms of both relative velocities of the collision partners and center of mass frame. In crossed-molecular beam experiments, the angular distribution of the scattered product is contained in the Newton sphere whose center is the center of mass of the colliding species. We are able to tell the angular distribution of the scattered products by defining the products as either forward, sideways or backwards as illustrated in the example shown in figure 1.0. The figure illustrates the angular scattering distributions with the Newton diagram superimposed on it.

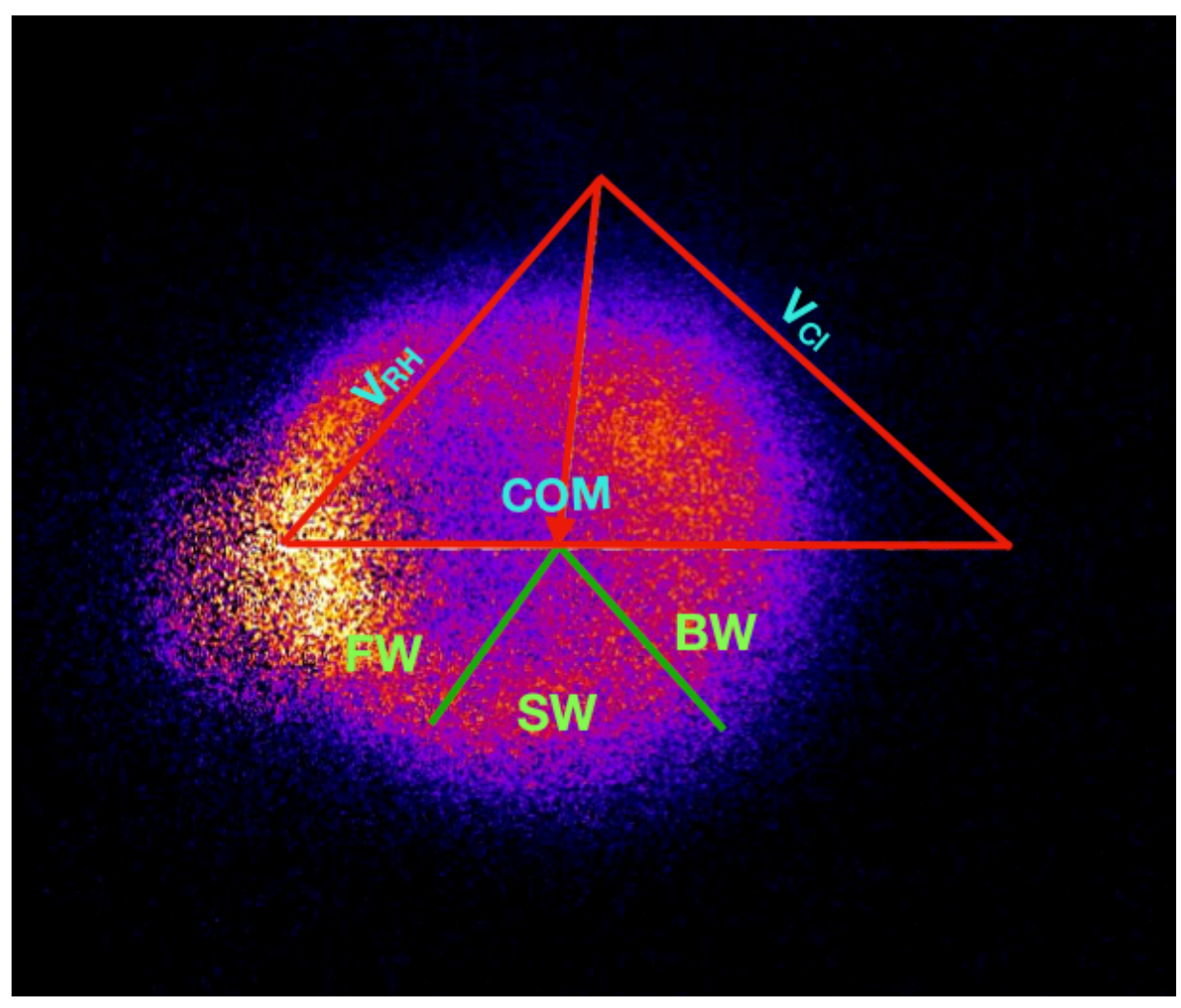

Figure 1.0 Center-of-mass distribution of the $\mathrm{C}_{4} \mathrm{H}_{7}$ radical from the reaction between $\mathrm{C}_{4} \mathrm{H}_{8}$ and $\mathrm{HCl}$.

Lab frame measurements such as speed and angles can be transformed to the center of mass frame by using geometric relationship between the velocity vectors (Newton 
diagram) which provides a kinematic analysis of the reaction as shown in Figure 1.1. In this figure, the velocities in the laboratory frame are represented by $v_{1}$ and $v_{2}$ while the relative velocities in the center of mass frame are $\mathrm{u}_{1}$ and $\mathrm{u}_{2}$. The relation between the velocity vectors 1 and 2 in the center of mass and laboratory frame is expressed as

$$
\begin{aligned}
\mathbf{V}_{\mathbf{C M}} & =\frac{m_{1} v_{1}+m_{2} v_{2}}{M} \\
\mathbf{U}_{\mathbf{1}} & =\frac{m_{2}}{M} v_{\text {rel }} \\
\mathbf{U}_{\mathbf{2}} & =\frac{m_{1}}{M} v_{r e l}
\end{aligned}
$$

The relative velocity, $\mathbf{V}_{\text {rel }}$ is expressed as

$$
\mathrm{V}_{\text {rel }}=\mathrm{u}_{1}-\mathrm{u}_{2}=v_{1}-v_{2}
$$

The angle between the relative velocity vectors, $(\theta)$ is center of mass scattering angle and the relation between the center of mass speed of the scattered products to their relative velocity is given by

$$
\begin{aligned}
& \mathbf{U}_{\mathbf{1}}=\frac{m_{2}}{M} V^{\prime} r e l \\
& \mathbf{U}_{\mathbf{2}}=-\frac{m_{1}}{M} V^{\prime} r e l
\end{aligned}
$$




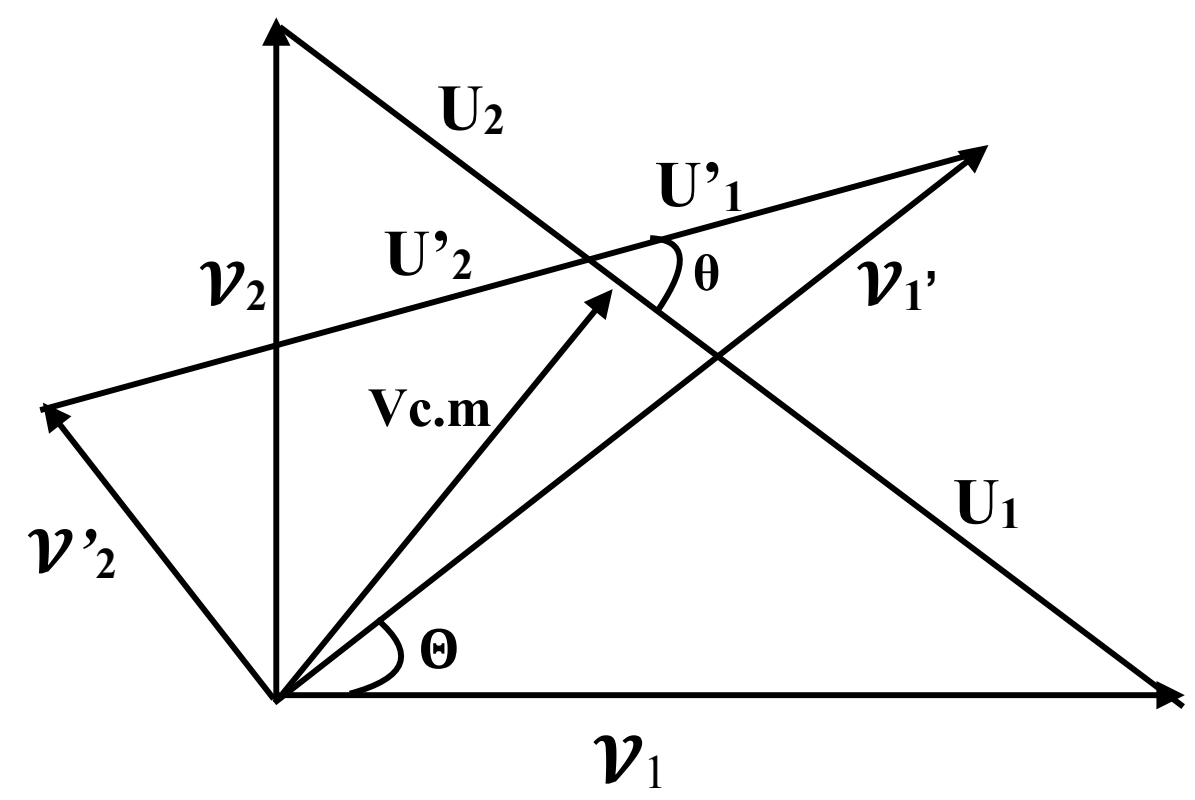

Figure 1.1. Newton diagram showing the relation between the laboratory and center of mass velocities. The laboratory frame is denoted by v and the center-of-mass frame is denoted by $\mathrm{u} . \boldsymbol{\Theta}$ and $\theta$ are the deflection angles in laboratory and center-of-mass frames respectively.

The use of DC slice imaging technique, described in chapter 2.4, has the advantage of directly providing the translational energy and the angular distribution without any inversion methods. The evolution of theoretical calculations have given us much more insight with the ability to explore the potential energy surface to give us better understanding of the reaction dynamics.

My dissertation discusses two main detection techniques in probing scattered products. The first section involves using state resolved detection to probe inelastic scattering of vibrationally excited $\mathrm{NO}(v=1)$ with $\mathrm{Ar}$, and the second part involves using universal detection to probe reactive scattering of $\mathrm{O}\left({ }^{3} \mathrm{P}\right)$ with a series of target systems 
(Amines and Alcohols). The outline of the thesis is structured as follows: Chapter 2 focuses on the experimental techniques used in these studies. An overview of crossedmolecular beam, ion imaging, universal detection, resonance enhanced multiphoton ionization (REMPI) will be discussed. The study of the collisions of $\mathrm{NO}\left(\mathrm{X}^{2} \Pi\right)$ with $\mathrm{Ar}$ reactions has been the benchmark in studying rotational energy transfer. Chapter 3 of the thesis describes results of experiments using our crossed-beam DC slice ion imaging apparatus to image the inelastic scattering of vibrationally excited $\mathrm{NO}(\mathrm{v}=1)$ with Ar. Chapter 4 of the thesis reports the use of single photon ionization to investigate the reaction dynamics of the reaction between $\mathrm{O}\left({ }^{3} \mathrm{P}\right)$ with Amines and the role of intersystem crossing on the singlet-triplet surface of the $\mathrm{H}$ - abstraction mechanism. Theoretical calculations are also carried out to get a better understanding of the reaction mechanism. Oxygenated systems such as alcohols for a very long time have been of great interest to experimentalists and theoreticians because of the very important roles the play in combustion and atmospheric chemistry. These systems offer special dynamical interest in understanding the mechanism of these reactions with $\mathrm{O}\left({ }^{3} \mathrm{P}\right)$. Chapter 5 deals with understanding the mechanism of $\mathrm{H}$-atom abstraction of $\mathrm{O}\left({ }^{3} \mathrm{P}\right)$ radicals with n-propanol and iso-propanol using universal detection to probe the scattered hydroxyalkyl products. Collision energy dependence studies were performed to the two different types of alcohols under study. 


\section{CHAPTER 2}

\section{EXPERIMENTAL METHODS}

\subsection{Crossed Molecular Beams}

Molecular beams are produced by allowing a gas at higher pressure to expand through a small orifice, and with the help of a skimmer are collimated into an evacuated chamber. This results in the production of a beam of particles (radicals, atoms, molecules or ions) moving with almost the same velocities and with few collisions occurring between them. Generally, the gas expansion is characterized by the Knudsen number which is expressed as $K_{\mathrm{n}}=\lambda / d$, where $\lambda$ is the mean free path of the molecules in the gas reservoir and $\mathrm{d}$ is the diameter of the nozzle. For $K_{\mathrm{n}} \geq 1$, the molecules do not collide frequently in the nozzle vicinity and the molecular beam generated is termed an effusive beam. Effusive beams normally have low intensities that are insufficient for scattering experiments. This type of beam is characterized by a broad Maxwell-Boltzmann distribution. But if the Knudsen number satisfies $K_{n} \ll<1$, a supersonic condition can be achieved resulting in fast molecular beams with narrow velocity distributions, high density, and small angular divergence. If $\lambda \ll<$, many collisions occur during the expansion thereby establishing a local equilibrium at each point along the expansion and at this point it is treated as an adiabatic process. The molecules, which are accelerated by the pressure difference, reach the local sonic speed at the nozzle throat and then as the local temperature (and speed of sound) drops the term supersonic expansion becomes appropriate. The total energy available per molecule in the gas container is converted into kinetic energy and the rotational temperature of the beam becomes very close to the low 
translational temperature. A typical free jet expansion is illustrated schematically in Fig. 2.0

In this way a stream of gas molecules can be directed towards a specific target. Molecular beams allow the study of a system that is far away from equilibrium due to the fact that the translational and internal energy of the molecules in the beam can be tuned independently. Atomic and molecular beams for decades have been very useful to experimentalists in probing chemical reactivity ${ }^{4-8}$ and molecular structures. ${ }^{9}$ One can vary both the conditions under which a molecular beam is formed and the composition of the gas mixture in order to have control of the most probable speed of the atoms, degree of clustering resulting from the expansion of the beam, ${ }^{10}$ and the effective temperature of the molecules in the beam. ${ }^{11}$ By using a small amount of molecules of interest seeded in a light carrier gas, we are able to generate supersonic beams with very low rotational temperature thereby converting the enthalpy of the gas to bulk translational energy. ${ }^{12}$ Very low gas density characterizes molecular beams hence the need to propagate them through a medium that does not disturb them over the length scale of the experiment. The mean free path of the propagation beam has to be greater than the distance from the collision region to the detector.

Crossed-beam collisions exploit the well-defined directionality to enable the experimentalist to measure the angular distribution of atoms or molecules in one beam that have been scattered from those in the other beam and often reveals very important information such as the interaction potentia $1^{13}$ and dynamics of the reaction. 


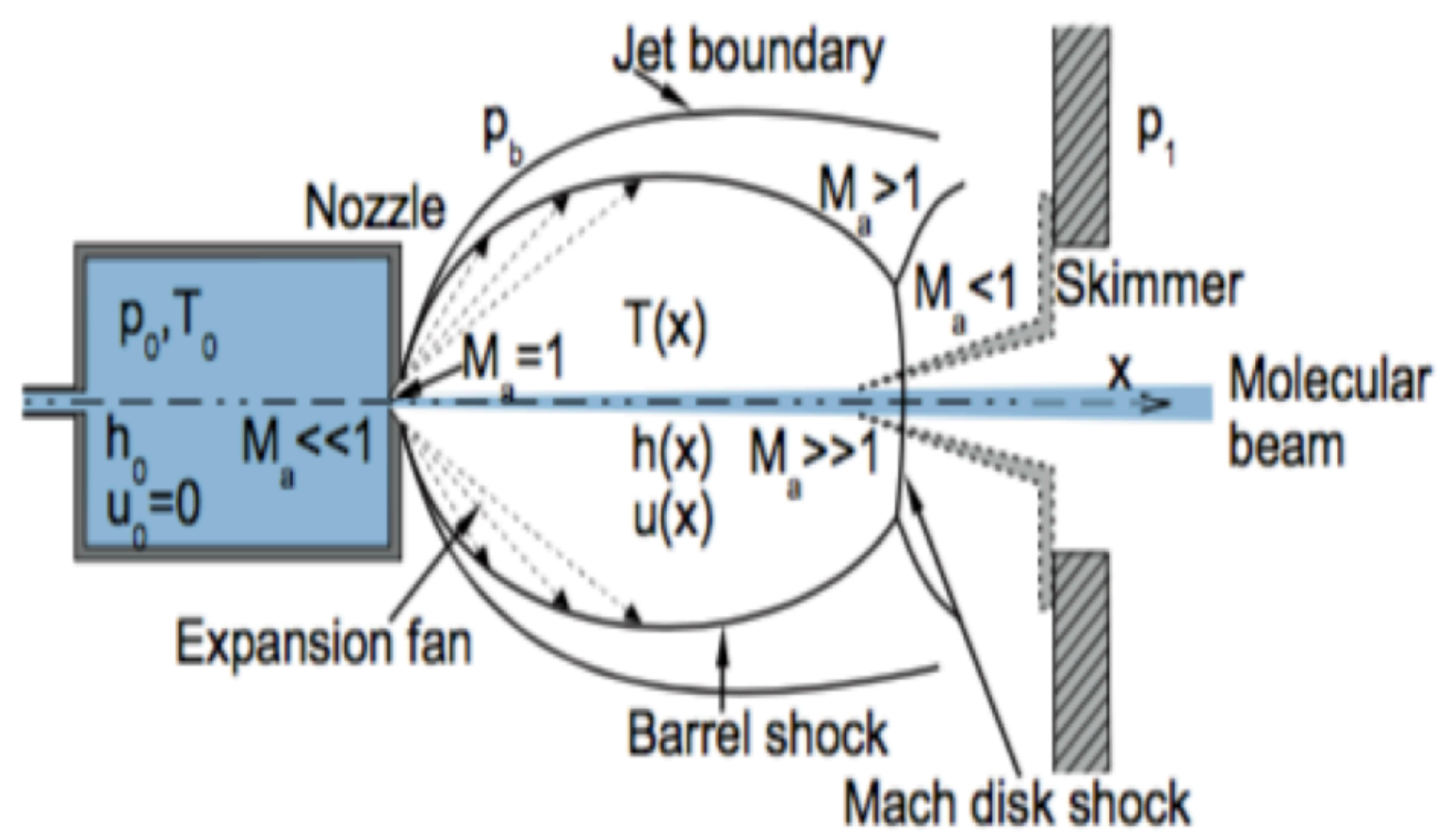

Figure 2.0: Schematic illustration of supersonic expansion of a gas through a nozzle adapted from "molecular dynamics in free clusters and nanoparticles studied in molecular beams" by Michal Farnik, 2011.

\subsection{Universal Detection}

During the late 1960s electron bombardment ionization ${ }^{14-15}$ became the main technique used as 'universal detection' in probing products in molecular beam spectroscopy but its downfall has been the huge interfering background signal that undermined certain important dynamic features of interest as well as having to endure 
very long acquisition time. ${ }^{16-17}$ In an attempt to overcome this limitation, Lee and coworkers ${ }^{18}$ used differential pumping in both the source and detection region of their chamber and it drastically reduced the background interference and enhanced the detection sensitivity in the ionization region although issues about fragmentation of parent species in producing daughter ions still remain a big problem. Single photon ionization usually occurs in the vacuum ultraviolet (VUV) region and has the capability of performing soft ionization of basically all organic compounds. The development of VUV for single photon ionization has been limited by the availability of VUV light sources. In the early 1990, a high-resolution monochromatized third-generation VUV synchrotron source at the chemical dynamics beamline was established. In addition to its universality, the ability to tune the radiation offers it an added advantage of selectivity in the internal energy of the detected species. The first attempt to couple VUV photoionization detection to crossed-molecular beam was carried out by Suits et al. ${ }^{19}$ in Berkeley by using the synchrotron radiation source at the Advanced Light Source to study reactive scattering of chlorine with propane. There are very limited numbers of synchrotron light sources in the world. However, the need for researchers to locate at one of the world's few source facilities with a VUV synchrotron source is a big challenge. Recently, inspired by synchrotron studies, Casavecchia and coworkers were able to use low energy electrons to probe products in their crossed-beam scattering experiments by tuning the electron energies below that of the dissociative ionization. This reduces fragmentation of the parent species. ${ }^{20-22}$ Over the past few decades there has been major achievement with the production of commercial available inexpensive lasers such as the $157 \mathrm{~nm} \mathrm{~F}$ (7.9 eV) excimer laser. This gives 10-ns-long pulses with the capability of 
producing more than $1 \mathrm{~mJ}$ per pulse at a repetition rate up to $100 \mathrm{~Hz}$. Davis and his group has utilized the $157 \mathrm{~nm}$ excimer laser to study reactions between transition metal atoms with hydrocarbon molecules with very high signal to noise ratio compared to electron impact ionization. ${ }^{23-25}$ The Suits group has used of $157 \mathrm{~nm}(7.9 \mathrm{eV})$ to carry out single photon ionization of alkyl radicals generated form crossed-beam imaging studies of reactions between chlorine and fluorine radicals with very high efficiency due to the fact that, the ionization potential of most of these radicals is below $7.9 \mathrm{eV} .{ }^{26-29}$

\subsection{Resonance Enhanced Multiphoton Ionization.}

Resonance-enhanced multiphoton ionization (REMPI) is a unique soft photoionization technique that has been used extensively as a selective ionization probe for mass spectrometry. REMPI is a powerful spectroscopic technique, which involves ionization of atoms or molecules by successive absorption of two or more laser photons. This ionization technique relies on the tunability of lasers to ionize molecules with rotational and vibrational state selectivity. In order to achieve ionization using REMPI, the sum of the absorbed photon energy should exceed the ionization energy of the targeted species. The wavelength of the excitation laser can be varied such that a photon can ionize the molecule when it is on resonant with single or multiple photon absorption tuned, exciting the molecule to an intermediate electronic state. Because of a resonance state condition, the multiphoton absorption intensity is enhanced to several orders of magnitude compared to the non-resonant ionization. Various excitation schemes can be used for REMPI, and they are described by the number of photons used for excitation and ionization. For example, in the simplest resonance-ionization scheme one photon is used 
to excite the molecule and an additional photon to ionize it, this is known as the $(1+1)$ REMPI scheme, also sometimes termed resonant 2-photon ionization or R2PI. The first photon excites the molecule to a higher energetic intermediate state. If the lifetime of the intermediate state is sufficiently long, a second photon is absorbed and elevates the molecule above the ionization threshold. Sometimes, the intermediate state is at an unfavorably high energy for the available laser wavelength and thus can only be reached by absorbing two or even three photons; ionization, subsequently, must be completed by absorbing an additional photon (2+1 and 3+1 REMPI, respectively). Figure 2.1 illustrates ionization REMPI schemes for $1+1$ and 2+1 REMPI. Higher order REMPI schemes usually require high photon fluxes to ensure maximum absorption of all photons during the short life of the intermediate state and this causes more fragmentation compared to the 1+1 REMPI scheme. Zare and coworkers performed the first studies on reaction dynamics of $\mathrm{Ba}+\mathrm{HCl}$ using multiphoton ionizationn of $\mathrm{BaCl} \cdot{ }^{30}$ Further advancement of REMPI enabled Houston and Chandler ${ }^{31}$ to famously image methyl from the photodissociation of methyl iodide. In their experiment, $\mathrm{CH}_{3} \mathrm{I}$ in pulsed molecular beam was dissociated by $266 \mathrm{~nm} \mathrm{Nd:YAG} \mathrm{laser,} \mathrm{and} \mathrm{the} \mathrm{CH}_{3}$ photofragment was ionized by 2+1 REMPI at $330 \mathrm{~nm}$. 

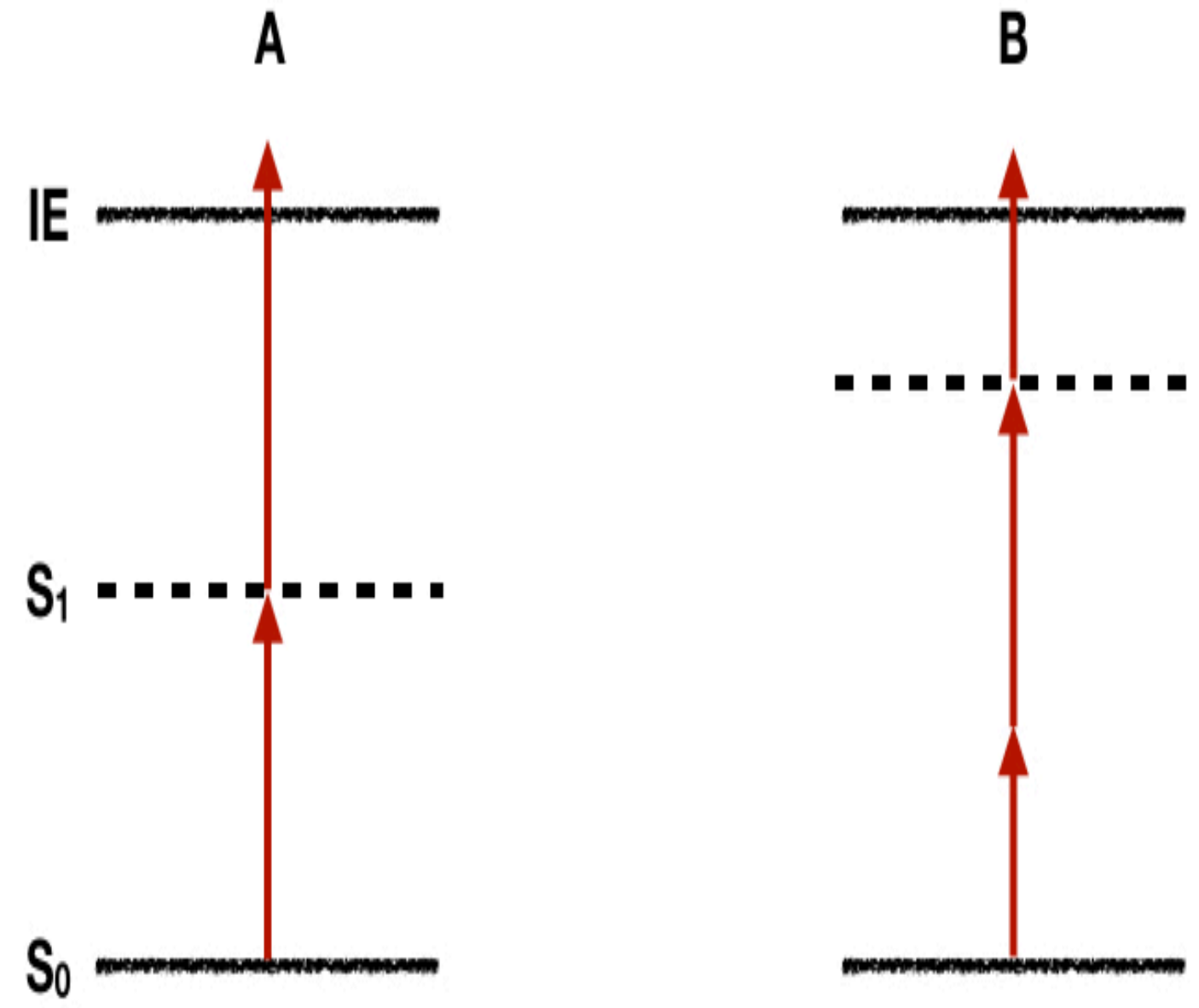

FIGURE 2:1 Schemes for ionization by resonance-enhanced multiphoton ionization (REMPI) (A) Onecolor, two-photon (1+1) REMPI, (B) 2+1 REMPI 


\subsection{ION IMAGING}

The ion imaging technique allows the spatial distribution of a reaction product species to be visualized directly, yielding the entire three-dimensional angular and velocity distribution of a photolysis or reaction event in a single digital snap shot. Qualitatively, it also provides a direct visualization method of the chemical process. Chandler and Houston ${ }^{32}$ pioneered the foundation of the state of the art ion imaging technique being used today when they were investigating the photodissociation dynamics of methyl iodide. A typical ion imaging technique involves sending a molecular beam through a small hole of a repeller plate, where it is photodissociated by a photolysis laser. The resulting product molecule is then ionized with a probe laser. The second plate, the extractor, is used together with the repeller to maintain an electric field and direct the three-dimensional ion cloud formed along a time-of-flight tube on to a two-dimensional micro-channel plate (MCP) which is coupled with a fast phosphor screen. Ions striking the front face of the MCP gives rise to a burst of electrons at the back face, as these electrons strike the phosphor screen they produce a flash of light. The pattern of ions striking the MCP is transformed by this process into an image on the phosphor screen, which may can be captured by a CCD camera, digitized and sent to a computer for processing and analysis. A tedious mathematical inversion method (Abel transformation) is often used to reconstruct the $3 \mathrm{D}$ distribution in order to extract meaningful information of the 2D projected image. All information (kinetic energy, and angular distributions) is extracted from the spatial appearance of the two-dimensional image, which gives us direct information on the dynamics of the photodissociation event. A major drawback of 
this technique was the poor resolution and distorted image acquired due to the use of grids in the aperture of the electrodes. Figure 2.2 shows a pictorial representation of a conventional ion imaging apparatus. In an attempt to overcome this problem Eppink and Parker $^{33}$, introduced the velocity map imaging (VMI) in which they removed the grids and instead used the plates as focusing electrodes. This allowed particles with the same initial velocity vector to be mapped onto the same point of the detector irrespective of their point of origin. The lens can also be used to magnify the resulting ion image, although there is the need to calibrate the lens to determine the magnification factor. The magnification increases the energy resolution as the spatial separation of the rings appearing on the detector is increased.

Despite the improvement achieved on the energy resolution, the projectionreconstruction requirement still existed. The use of the inversion process introduces noise in the symmetry axis of the recovered image thereby compromising experimental resolution. The VMI method also requires the system to have cylindrical symmetry in order to retrieve the 3D distribution of the 2D velocity projections. This requirement of the VMI method limits its use particularly when undertaking polarizations experiments. The introduction of slicing was aimed in addressing these challenges. In principle, the central slice of the ion cloud contains the full angular and velocity distribution thereby eliminating need for the Abel transformation method for reconstruction of the image. Kitsopoulus et al. ${ }^{34}$ used delayed pulsed extraction to expand the ion cloud followed by field free expansion to detect the central slice of the ion cloud. The drawback to this technique was the poor resolution of the image due to the introduction of fine mesh on the ion optics assembly and space charge between the ions. The Suits group ${ }^{7}$ introduced 
Direct Current (DC) Slice Imaging that addressed the major drawback of the slicing technique of Kitsopoulus et al. and permitted VMI conditions to be maintained. The use of grids or pulsed electric field was eliminated and replaced with an additional lens at low voltages to stretch the ion packet in the acceleration region, increasing the arrival time spread onto the detector. This enables the central slice of the ion cloud that contains the translational energy release and the angular distribution to be imaged directly onto the MCP detector. At the same time this led to an additional 10-fold enhancement in velocity focusing compared to standard VMI.

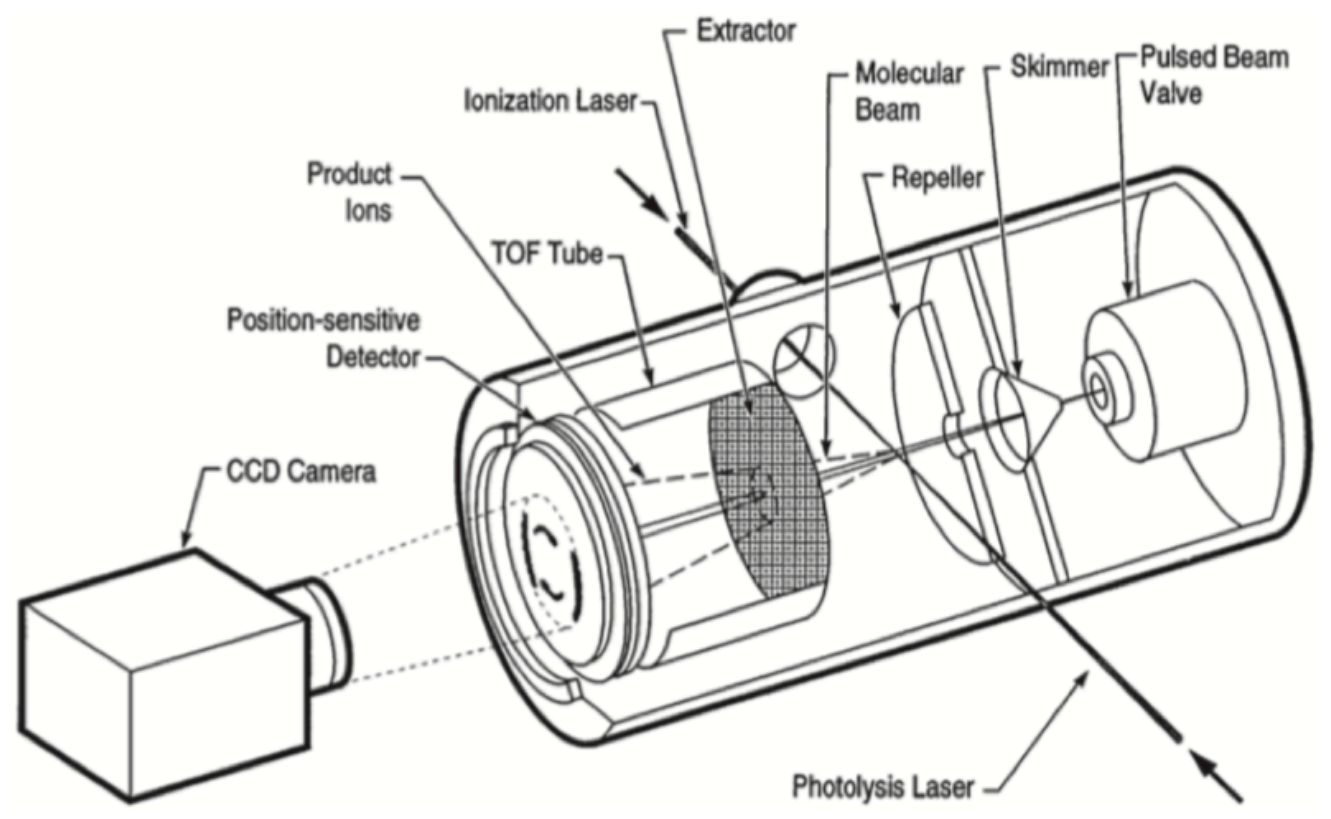

Figure 2.2 Set-up diagram of a conventional photofragment ion imaging apparatus. Diagram adapted from McDonnell et al, J Mass Spectrum. 333: 415. John Wiley and Sons. Copyright 1998. 


\subsection{INSTRUMENT SET-UP}

The experiments presented here were carried out in our crossed-beam imaging apparatus as shown in Fig.2.3. The make up of the machine consists of two perpendicular source chambers that are fixed at $90^{\circ}$, and a reaction chamber. The reaction chamber is differentially pumped to a base pressure of $10^{-8}$ torr to ensure single-collision conditions while the source chambers' pressures are maintained at $10^{-7}$ torr (base) and operated to a pressure of $10^{-5}$ torr (beams on) pumped by turbomolecular pumps mounted under each chamber. The radical beam and our target molecular beam are isolated in separate source chambers, and are seeded in various carrier gases $\left(\mathrm{H}_{2}, \mathrm{He}, \mathrm{Ar}\right.$,) to vary the collision energy. Piezoelectric actuators (stack) and piezoelectric disc valves were pulsed at $10 \mathrm{~Hz}$ to generate the molecular beam and after supersonic expansion, beams are skimmed into the main reaction chamber. The scattered radical products are immediately ionized after collision in the interaction region with either a VUV excimer laser $\left(\mathrm{F}_{2}, 7.9 \mathrm{eV}\right)$ or by employing a resonance enhanced mulitphoton ionization (REMPI) detection scheme. The product ions are accelerated via a four electrode dc slice ion optics assembly to impact on a $75 \mathrm{~mm}$ diameter dual micro-channel plate (MCP) detector coupled to a fast phosphor screen held at $5 \mathrm{KV}$. The front of the MCP assembly is held at ground potential and the back plate is pulsed to "gate" the central slice of the reaction scattered products at a specific mass by an application of a high voltage pulse (2.2 - $1.2 \mathrm{kV}$ bias, $1200 \mathrm{~ns}$ width) using a commercial pulser (DEI PVX-4140). The timing of the pulsed molecular beam, firing of the photolysis and probe lasers, and the detector gate pulse were controlled using a delay generator (BNC 555). The resultant images are recorded using a charged coupled device camera. The dc slice imaging detection scheme and megapixel acquisition 
program (NUACQ-2) were used to accumulate the raw images containing centroided data. Background subtraction and density-to-flux corrections were performed prior to transforming the scattered distributions in the center of mass frame. ${ }^{35}$ 


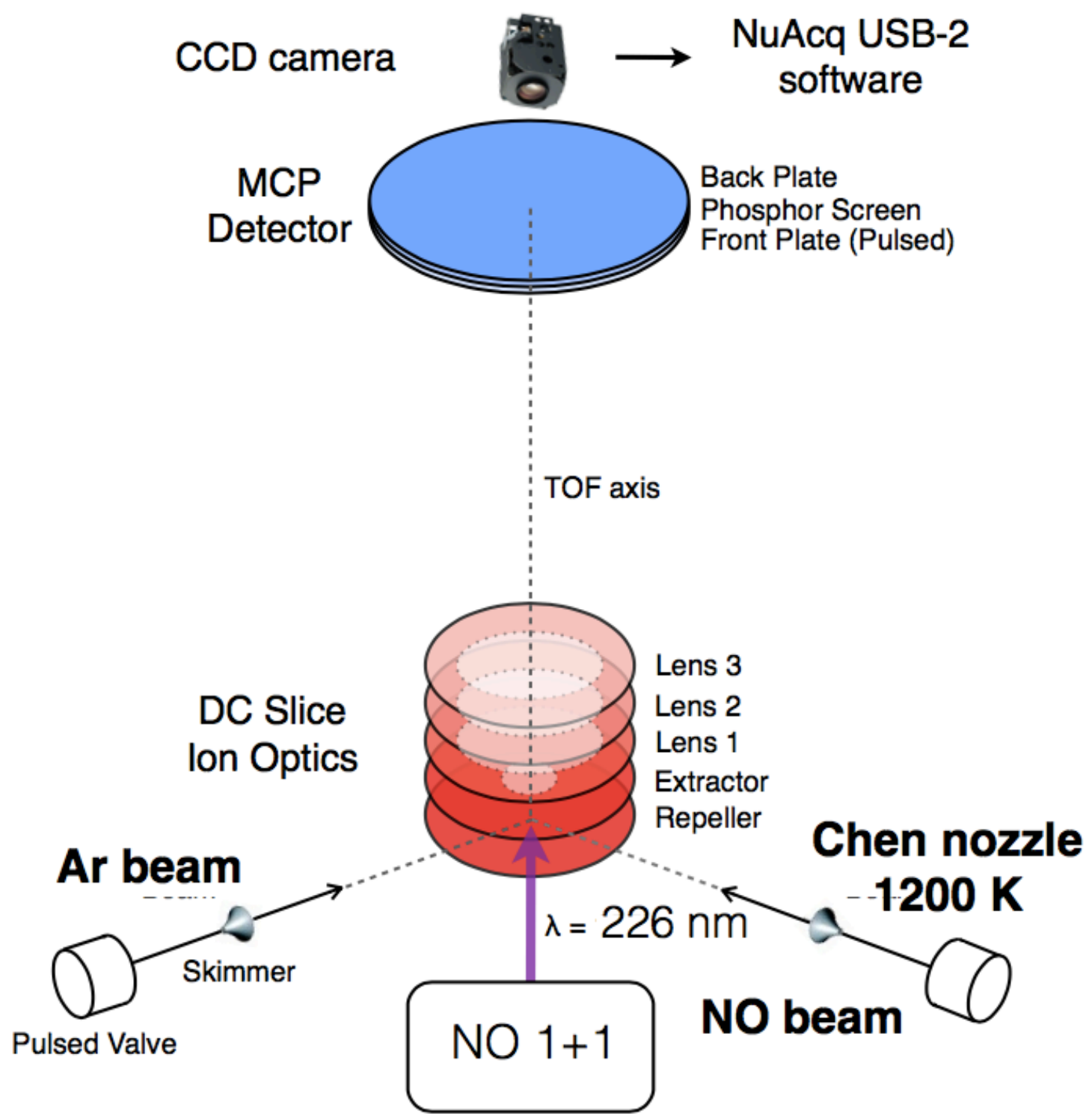

Figure 2.3: Schematic view of the crossed-beam apparatus combined with universal sliced dc ion imaging. 


\subsection{Piezo Stack Valve}

Proch and Trickl famously employed piezoelectric disk actuators with a high voltage switch to develop a valve for a molecular beam source. ${ }^{36}$ Although these valves have short opening times, they still exhibited some shortfalls such as long pulse durations, and low gas throughput. One very important limitation of disk actuators is its low acceleration force, which has a maximum value of only $10 \mathrm{~N}$. This limits the ability of the valve to open large nozzles at higher pressures. Over the last few years our lab has developed a new, robust valve ${ }^{37}$ based on piezoelectric stack actuators which was inspired by an earlier design of Gentry et. ${ }^{38}$ Our new design valve shows a better improvement in the pulse duration and molecular densities compared to the disk translators. With our new design we are able to achieve up to $180 \mu \mathrm{m}$ linear motion of the plunger with pushing and pulling force up to $4500 / 500 \mathrm{~N}$ respectively.

Figure 2.4 shows a schematic view of the valve assembly. It operates through the application of a high voltage pulse to the actuators. In its closed position, a high voltage of $+800 \mathrm{~V}$ is applied from a high voltage power supply, which delivers an average power of $200 \mathrm{~W}$ (Kepco BHK 1000-0.2MG). To open the valve, the actuator is grounded by switching the actuator voltage to $0 \mathrm{~V}$ with a fast high voltage transistor switch (Behlke HTS 61-03 GSM) or a DEI pulse generator (pvx-4140) which is combined with a highcapacitance $\mathrm{RC}$ circuits $(\mathrm{R}=1 \Omega, \mathrm{C}=20 \mu \mathrm{F})$.

We have recently been able to generate a high-density oxygen radical source from $\mathrm{SO}_{2}$ by attaching a $10 \mathrm{~mm}$ long cylindrical quartz tube with an inner diameter of $1 \mathrm{~mm}$ to the end of the nozzle of the valve which confines the molecules along the length of the 
quartz tube. This enables us to loosely focus a $193 \mathrm{~nm}$ excimer laser beam along the quartz tube to initiate photolysis of $\mathrm{SO}_{2}$. This generates an intense oxygen beam with the oxygen atom population density increasing by a factor of 3 compared to when we do not have a quartz tube attached to the exit of the nozzle. Figure 2.4 is a schematic view of the valve assembly without the attached quartz tube.

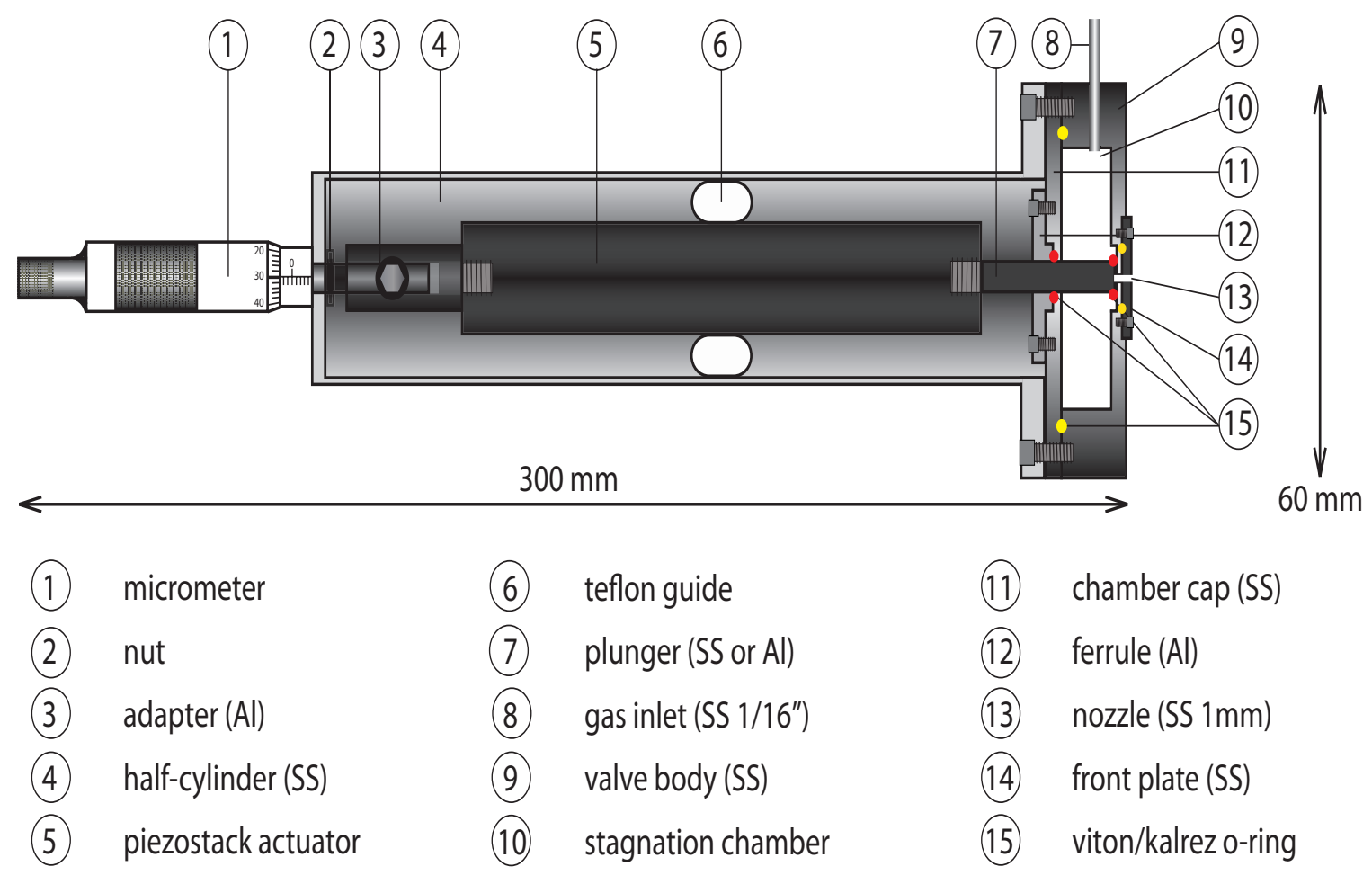


Figure 2.4: Schematic view of the valve assembly. The piezostack actuator is mounted and aligned outside of a stainless steel valve body in a half-cylinder. The valve is sealed by a kalarez o-ring (yellow spots) both at the tip and at half-length of the plunger. Other o-rings are shown in red. The teflon guide is used to to form a snug fit that restricts lateral movement of the piezo actuators and prevents any torqu. The fine micrometer head is used to regulate the volume of gas through the orifice. 


\title{
CHAPTER 3
}

Imaging the inelastic scattering of vibrationally excited $\mathrm{NO}(v=1)$ with Ar.

\author{
Alexander Kamasah ${ }^{1}$, Hongwei Li ${ }^{1}$, Jolijn Onvlee ${ }^{2,3}$, Ad van der Avoird ${ }^{3}$, \\ David H. Parker ${ }^{4}$, and Arthur G. Suits ${ }^{1, *}$ \\ ${ }^{1}$ Department of Chemistry, University of Missouri Columbia MO 65211 \\ ${ }^{2}$ Center for Free-Electron Laser Science, Deutsches Elektronen-Synchrotron DESY, \\ Notkestrasse 85, 22607 Hamburg, Germany \\ ${ }^{3}$ Theoretical Chemistry and ${ }^{4}$ Department of Laser Physics, Institute for Molecules and \\ Materials, Radboud University, Heyendaalseweg 135, 6525 AJ Nijmegen, The \\ Netherlands
}




\begin{abstract}
The inelastic scattering of vibrationally excited $\mathrm{NO}(v=1)$ with Ar at a collision energy of $3.0 \mathrm{kcal} \mathrm{mol}^{-1}$ was investigated in crossed beams. Vibrationally excited NO was generated by flash heating and rotationally cooled by the supersonic expansion. The differential cross sections were compared to those of the vibrational ground state $\mathrm{NO}(v=$ 0) with Ar, which were also compared to theoretical calculations for scattering from the ground vibrational level. The differential cross sections show a similar strong $j$ dependence of the rotational rainbow maxima from the inelastic scattering for both NO $(v$ $=0)$ and $(v=1)$ but no significant differences between $\mathrm{NO}(v=0)$ and $(v=1)$ were seen.
\end{abstract}




\subsection{INTRODUCTION}

Inelastic scattering of the open-shell radical NO with rare gases has been a benchmark for understanding collision dynamics for decades, and new aspects continue to be revealed. Nitric oxide is chemically stable and spectroscopically convenient, and has been the target system of choice for experimental studies of $\mathrm{NO}^{2} \Pi$ collision dynamics and energy transfer mechanisms. The ${ }^{2} \Pi$ ground state of NO follows Hund's case (a) when the rotational quantum number $\mathrm{j}$ is small. The spin and orbital angular momentum interact with each other and they form two spin-orbit components with $\Omega=1 / 2$ and $3 / 2$. The lower of the two-orbit levels is noted $F_{1}$ and the upper $F_{2}$; in the low $\mathrm{j}$, case (a) limit, $F_{1}$ is $\Omega=1 / 2$ and $F_{2}$ is $\Omega 3 / 2$. The splitting between the two levels is about $125 \mathrm{~cm}^{-1}$. In addition to the spin orbit-coupling, each rotational level is further split into doublet because of the coupling between the nuclear rotation and the electronic orbital angular momentum. This doubling is termed $\Lambda$-doubling and spectroscopically, the notation used for these levels is $e$ and $f$ known as the total parity as shown in figure 3.1 . 


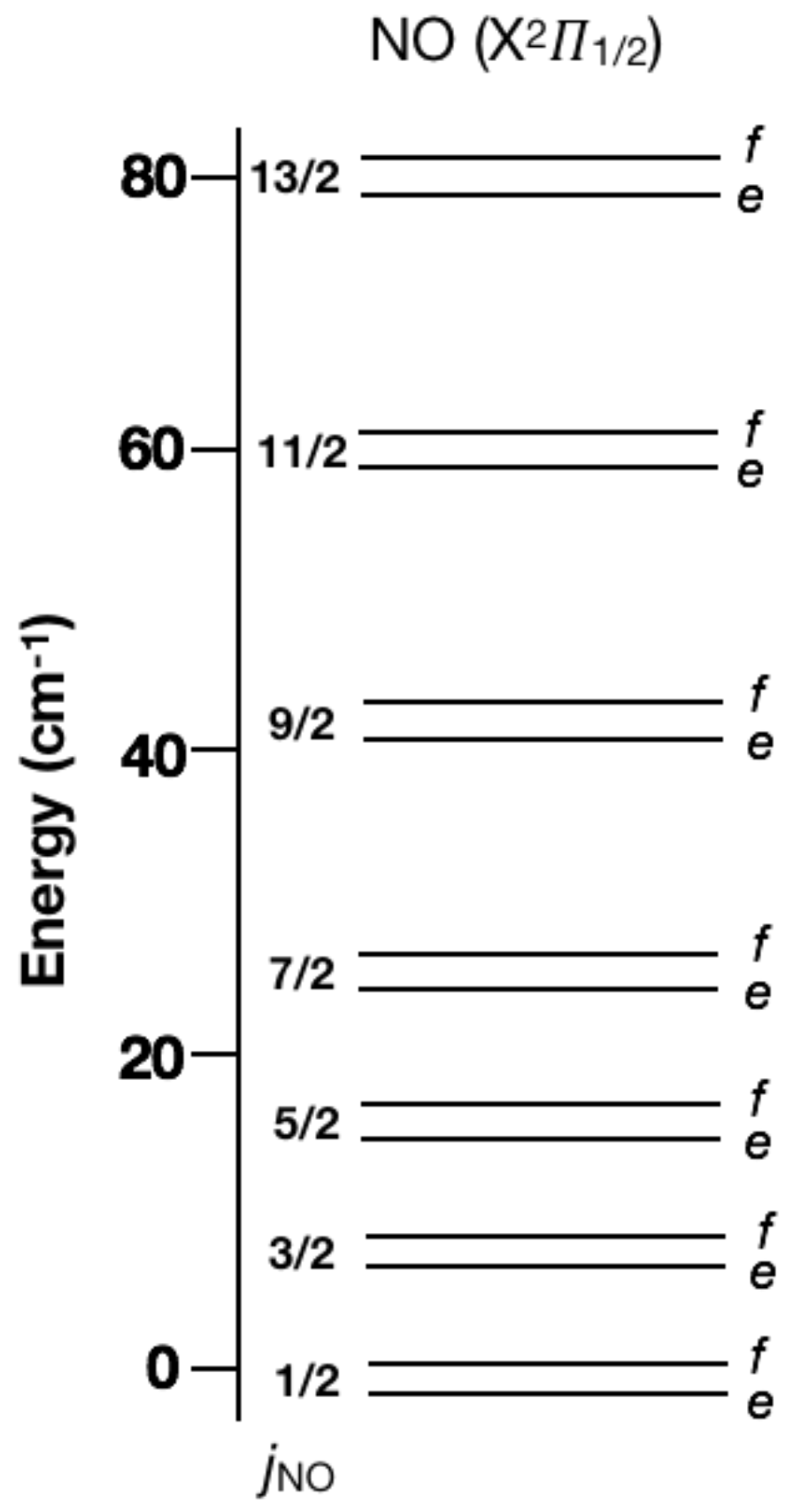

Figure 3.1 Energy level diagram of NO showing the energy splitting between the $\Lambda$-doublet components of each rotational level. 
Measurements of angular distributions from the scattering experiments directly embody the differential cross sections (DCSs), providing a sensitive probe of the molecular interaction potential energy surfaces (PESs). The application of velocity map imaging combined with crossed-molecular beams has been found to be very powerful in studies of reactive and inelastic scattering to understand the underlying dynamics ${ }^{2-33-39}$ Houston and coworkers first employed imaging to probe the state-to-state DCSs for inelastic collisions of ground state NO $\left(X^{2} \Pi\right)$ with $\mathrm{Ar}^{40-41}$ They observed rotational rainbow peaks in the angular distributions and these peaks move to backward scattering angles with increasing final rotational level. This was followed by work from the Suzuki, ${ }^{42}$ and Chandler groups ${ }^{43}$ in which fully state-resolved DCSs for the inelastic scattering of NO $\left(X^{2} \Pi, v=0, \mathrm{j}=0.5\right)$ with Ar were measured resulting from spin-orbit conserving and changing transitions. These arise from scattering from sum and difference potentials built from PESs, of $\mathrm{A}^{\prime}$ and $\mathrm{A}^{\prime}$ character. Their findings were consistent with quantum mechanical close-coupling (QM CC) calculations based on ab initio coupled-cluster $\operatorname{CCSD}(\mathrm{T})$ and CEPA potential energy surfaces. Later, Stolte, Brouard and coworkers ${ }^{44-47}$ were able to resolve the $\Lambda$-doublet effects on the DCSs for NO $\left(X^{2} \Pi\right)$ inelastic collisions with Ar by coupling a hexapole to the crossed beam velocity map imaging apparatus. They observed different quantum interference structures on DCSs for total parityconserving and changing collisions. Rigorous quantum scattering calculations suggest that the heteronuclear nature of NO plays an important role in the DCSs for the parityconserving collisions, but less important for the parity-changing collisions. ${ }^{48-49}$ Other work by Cline and coworkers ${ }^{50}$ also revealed a high degree of directional preference for NO rotation during collisions with Ar. They reported that the NO product orientation 
varies with the deflection angle and for lower rotational states, the variation with deflection angle becomes oscillatory. More recently, van de Meerakker and coworkers in Nijmegen used a Stark decelerator to produce a well-defined and velocity-controlled NO radical beam that intersected with a conventional beam of rare gases. Using velocity map imaging $^{33}$, they observed diffraction oscillations in the DCSs from the inelastic scattering of NO radicals with the rare gases $\mathrm{He}, \mathrm{Ne}, \mathrm{Ar}, \mathrm{Kr}$, and $\mathrm{Xe}^{51-52}$ This phenomenon is in excellent agreement with high-level QM CC calculations based on the most accurate PESs.

Although NO inelastic scattering has long been of interest, most such studies have focused on the vibrational ground state and very little has been reported for vibrationally excited NO. The difficulty is very likely due to the challenge of preparing a vibrationally hot but rotationally cold NO molecular beam. Optical pumping has been used to generate the vibrationally excited NO by direct absorption of infrared radiation. ${ }^{53-55}$ The rate constants were then measured for $\mathrm{NO}\left(X^{2} \Pi, v=1,2,3\right)$ with selected gas molecules including Ar. The thermally averaged cross-sections were reported for total removal of NO from the particular rovibronic level $X^{2} \Pi_{1 / 2}, v=3, \mathrm{j}=0.5,3.5$, and 6.5 in collisions with Ar at various temperatures, 27, 44, 53 and $139 \mathrm{~K} .{ }^{53}$ Alternatively, the Houston group ${ }^{56}$ prepared vibrationally excited NO at the exit of the valve nozzle through a photoinitiated reaction between $\mathrm{O}\left({ }^{1} \mathrm{D}\right)$ and $\mathrm{N}_{2} \mathrm{O}$. Due to the nature of the reaction, they were only able to make vibrationally excited NO at $v=5$. They measured the DCSs for rotationally inelastic scattering $(\mathrm{j}=6.5,16.5$ and 20.5$)$ of vibrationally excited $\mathrm{NO}(v=5)$ from Ar for the first time by coupling crossed molecular beams with velocity map imaging. On comparing the results of $v=5$ to $v=0$, they observed a small shift in 
rotational rainbow maxima to smaller angles in $v=5$ compared to the ground state $v=0$. They concluded that the shift in the rainbow angle is a manifestation of the increased angular anisotropy during vibrational excitation.

In the present work, we prepared the vibrationally excited $\mathrm{NO}\left(\mathrm{X}^{2} \Pi, v=1\right)$ by resistively heating the nozzle of our valve. We present the results of our crossed-beam study on vibrationally excited NO $\left(\mathrm{X}^{2} \Pi, v=1\right)$ with Ar by acquiring images for the inelastic scattered NO products using our DC slice imaging technique. After background subtraction and density-to-flux correction, the differential cross sections for various $\mathrm{j}$ levels of NO $(v=1)$ are derived to unravel the underlying dynamics, and compared to that from the inelastic scattering of $\mathrm{NO}(v=0)$ with Ar under same conditions.

\subsection{EXPERIMENTAL SECTION}

The experiments are carried out in a crossed molecular beam apparatus combined with DC slice ion imaging, which has been previously described elsewhere ${ }^{57}$. The apparatus consists of one reaction chamber and two source chambers that are perpendicular to each other. The Ar beam is pulsed at $10 \mathrm{~Hz}$ with a backing pressure of 5.3 bar into one source chamber via a piezoelectric stack actuator (Physik Instrumente, $\mathrm{P} 212.80)$ valve $^{37}$ with $120 \mu \mathrm{m}$ poppet translation and $50 \mu$ s pulse duration. The vibrationally excited NO is generated with a home-built flash pyrolysis ${ }^{58}$ source closely related to the pyrolysis design by Chen and coworkers ${ }^{59}$. The NO beam is introduced into the second source chamber by pulsing a piezoelectric pulsed valve operating at $10 \mathrm{~Hz}$ with a backing pressure of 5 bar. The NO beam is expanded into the $\mathrm{SiC}$ nozzle and resistively heated to $\sim 1200 \mathrm{~K}$ with a DC power supply (supplying a current of $1.6 \mathrm{~A}$ at a 
potential of $17 \mathrm{~V})$. The vibrationally excited $\mathrm{NO}\left(\mathrm{X}^{2} \Pi, v=1\right)$ is prepared and then rotationally cooled to $\sim 20 \mathrm{~K}$ before entering into the interaction chamber to be intersected by the Ar beam at $90^{\circ}$. The final scattered $\mathrm{NO}\left(\mathrm{X}^{2} \Pi, v^{\prime \prime}=0\right.$ or 1$)$ products are then stateselectively ionized using $(1+1)$ resonance-enhanced multiphoton ionization (REMPI) near $226 \mathrm{~nm}$ via the $\mathrm{A}^{2} \Sigma^{+}\left(v^{\prime}=0\right.$ or 1) electronic state and detected with the DC slice ion imaging technique. The UV radiation near $226 \mathrm{~nm}$ is generated by the frequency tripling of the fundamental output of a pulsed dye laser (Sirah Cobra-Stretch, LDS 698 dye) pumped by the second harmonic of a seeded Nd:Yttrium aluminum garnet (Nd:YAG) laser (Quanta Ray, Spectra Physics). A four-electrode de slice ion optics assembly accelerates the ions in the direction perpendicular to the scattering plane. After passing through a $75 \mathrm{~cm}$ field-free flight tube, the $\mathrm{NO}^{+}$ions are velocity focused onto a dual microchannel plate (MCP)/phosphor screen coupled detector. The detector is gated for the center slice of the scattered product ions and resultant images are recorded using a charge-coupled device (CCD) camera and analyzed with our data acquisition program. The background signal from the NO beam alone is subtracted and density-to-flux correction is performed before further analysis to obtain the angular distributions of the rotationally inelastic scattering.

\section{THEORY SECTION}

We performed QM CC calculations to compute state-to-state cross sections for $\mathrm{NO}(\mathrm{v}=0)$ + Ar. A scattering program for open-shell diatom-atom scattering was used that was originally developed for $\mathrm{OH}+$ rare gas collisions ${ }^{60}$. The ab initio potential energy surfaces constructed by Alexander were used as input ${ }^{61}$. Using the renormalized 
Numerov method, we propagated the wave function on an equidistant grid in $\mathrm{R}$ from 3.5 to 50 Bohr with 1094 points, where $\mathrm{R}$ is the distance between the Ar atom and the centerof-mass of the NO molecule. We took all NO rotational levels up to $\mathrm{j}=25.5$ and all partial wave contributions up to a total angular momentum of $\mathrm{J}=200.5$ into account in the channel basis set. The scattering cross sections were computed for a collision energy of $3.0 \mathrm{kcal} \mathrm{mol}^{-1}$ and for initial NO rotational levels up to $\mathrm{j}=6.5$.

\subsection{RESULTS AND DISCUSSION}

In this present work, the NO products at various rotational $\mathrm{j}$ levels are detected after inelastic collisions of $\mathrm{NO}(v=0,1)$ with Ar at a collision energy of $3.0 \mathrm{kcal} \mathrm{mol}^{-1}$. The experimental scattering results for the ground state $\mathrm{NO}(v=0)$ with Ar were compared to the theoretical results. Further comparison was conducted on inelastic scattering of the vibrationally excited $\mathrm{NO}(v=1)$ to the ground state $\mathrm{NO}(v=0)$ under the same conditions. This study is directed to examining whether the different vibrational excitation of the diatomic molecule affects the angular distributions of the rotationally inelastic scattering under the same conditions. Superelastic scattering of NO $(v=1)$ giving $(v=0)$ product was not expected or observed, as it is a process of much smaller total cross section.

We first measured REMPI spectra for NO to characterize the NO molecular beam generated from the flash pyrolysis source. The 1+1 REMPI spectra of $A^{2} \Sigma^{+} \leftarrow X^{2} \Pi(0,0)$ and $(1,1)^{62-63}$ bands were obtained at two different energy regions, corresponding to two different prepared vibrational levels for $\mathrm{NO}, v=0$ and $v=1$, respectively (as shown in

Fig.3.2). The rotational band contour is simulated with known parameters ${ }^{64-66}$ under the 
rotational temperature of $20 \mathrm{~K}$ using the PGOPHER program ${ }^{67}$. The good agreement of the REMPI spectra for both $\mathrm{NO} v=0$ and $v=1$ between experimental and simulated results (as shown in Fig. 1) indicates that the generated NO beam is rotationally cold, ca. $20 \mathrm{~K}$. At this rotational temperature, only the first few rotational levels $(\mathrm{j}=0.5$ to 5.5$)$ are significantly populated for our NO molecular beam source at both $v=0$ and $v=1$, and these rotational levels thus dominate the inelastic collisions we observe with Ar. The final rotational states populated by inelastic scattering are ionized by $1+1$ REMPI employing main $\mathrm{R}_{11}$ branch (overlapping with the $\mathrm{Q}_{21}$ satellite branch) of the $\mathrm{A}^{2} \Sigma^{+} \leftarrow \mathrm{X}^{2} \Pi$ electronic transition. The vibrational ground state $\mathrm{NO} v=0$ is probed using the $(0,0)$ band, while the vibrationally excited state NO $v=1$ is probed using the $(1,1)$ band. The inelastic scattering images of the final rotational states for NO $v=0$ and $v=1$ are shown in Fig. 3.3 with the nominal Newton diagrams superimposed on them. The images portray the forward scattered signal pointing down (the direction of the NO beam) and the backward scattered signal pointing up. The background signal arises from NO in the molecular beam that does not undergo inelastic collisions with Ar. This background 

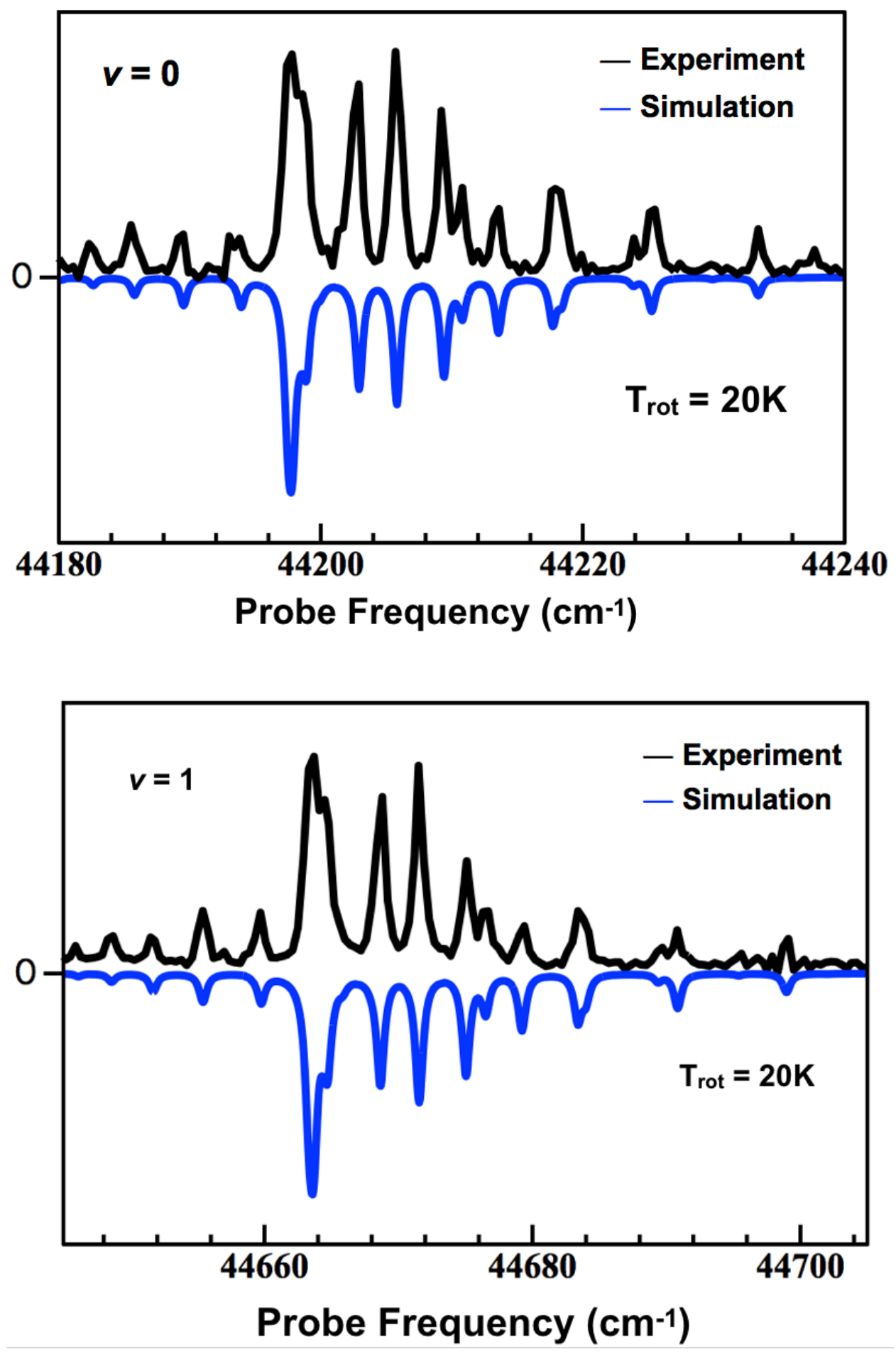
Figure $3.21+1$ REMPI spectra of NO recorded for $v=0$ (top panel) using $(0,0)$ band and $v=1$ (bottom panel) using $(1,1)$ band of the $\mathrm{A}^{2} \Sigma^{+} \leftarrow \mathrm{X}^{2} \Pi$ transition. The lower spectra (blue) are the simulation at $20 \mathrm{~K}$, whiles the upper spectra (black) are experimental results. 
is more intense for lower $\mathrm{j}$ levels because of the low rotational temperature $(\sim 20 \mathrm{~K})$ for our molecular beam. Therefore, the present study focuses on only high $\mathrm{j}$ levels, from 12.5 to 22.5 , in order to reduce the interference in the inelastic scattering from the background. 


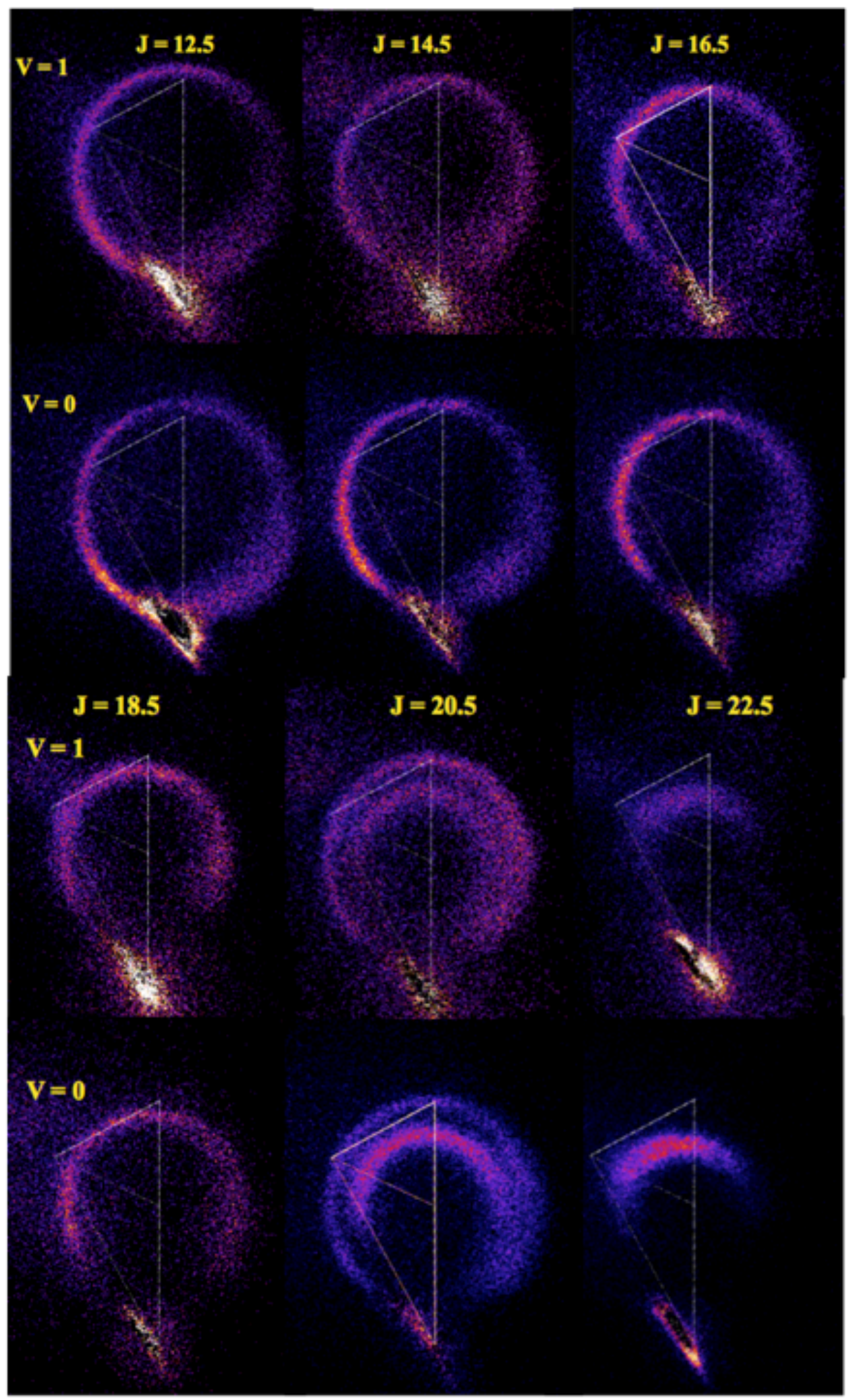


Figure 3.3. DC slice images for inelastic scattering of $\mathrm{NO}$ with Ar after background subtraction and density-to-flux correction at a collision energy of $3.0 \mathrm{kcal} / \mathrm{mol}$ with Newton diagram superimposed. First two upper panel compares the scattering images for $v=1$ to $v=0$, for $\mathrm{j}=12.5$, 14.5, and 16.5, whiles the Lower two panels compares also compares $v=1$ to $v=0$, for $\mathrm{j}=18.5$, 20.5 , and 22.5 . 
The images in Fig. 3.3 show a single sharp scattering ring for each $\mathrm{j}$ level except for $\mathrm{j}=$ 20.5 after the inelastic collision between $\mathrm{NO}$ and Ar. The size of the image decreases with increasing $\mathrm{j}$, which is consistent with the conservation of energy. The inner ring of the image labeled $j=20.5$ corresponds to signal at the $j=20.5$ level whereas the outer ring corresponds to $j=15.5$, since the $R_{11}(20.5)$ transition is very close to the $R_{21}(15.5)$ transition. Therefore, this image is analyzed to yield two separate DCS's. The center-ofmass angular distribution is obtained by integrating the signal over a certain radial region of interest to characterize the rotationally inelastic scattering. We first compared the experimental angular distribution of $\mathrm{NO}(v=0) / \mathrm{Ar}$ system with the theoretically predicted differential cross sections (DCS) for this system at different $\mathrm{j}$ levels. At present only the $v=0$ DCS is available for this system. In order to match our experimental condition of 20 $\mathrm{K}$ rotational temperature, each effective DCS distribution is generated by weighting the initial $\mathrm{j}$ states of $\mathrm{NO} v=0$ with a Boltzmann distribution at $20 \mathrm{~K}$.

Both experimental and theoretical DCS distributions (as shown in Fig. 3.4) of $\mathrm{NO}(v=0) / \mathrm{Ar}$ system exhibit the expected rotational rainbow peak that shifts to larger scattering angle with increasing rotational excitation. We observed forward scattering for $\mathrm{j}=12.5$ to 14.5 with the rotational rainbow maxima around $35^{\circ}$ and $50^{\circ}$ respectively. Sideways scattering was observed for $\mathrm{j}=15.5,16.5,18.5$, and 20.5 with the rotational rainbow maxima shifting to larger angles, and backward scattering was seen for our highest detectable $\mathrm{j}=22.5$ level with collision energy of $3.0 \mathrm{kcal} \mathrm{mol}^{-1}$. We see evidence of the double rainbow phenomenon as predicted by theory, with two rainbow peaks around $60^{\circ}$ and $120^{\circ}$ for $\mathrm{J}=15.5$ and 16.5 , despite averaging over the initial $20 \mathrm{~K}$ rotational distribution. This arises from scattering off either the $\mathrm{O}$ end or the $\mathrm{N}$ end of the 

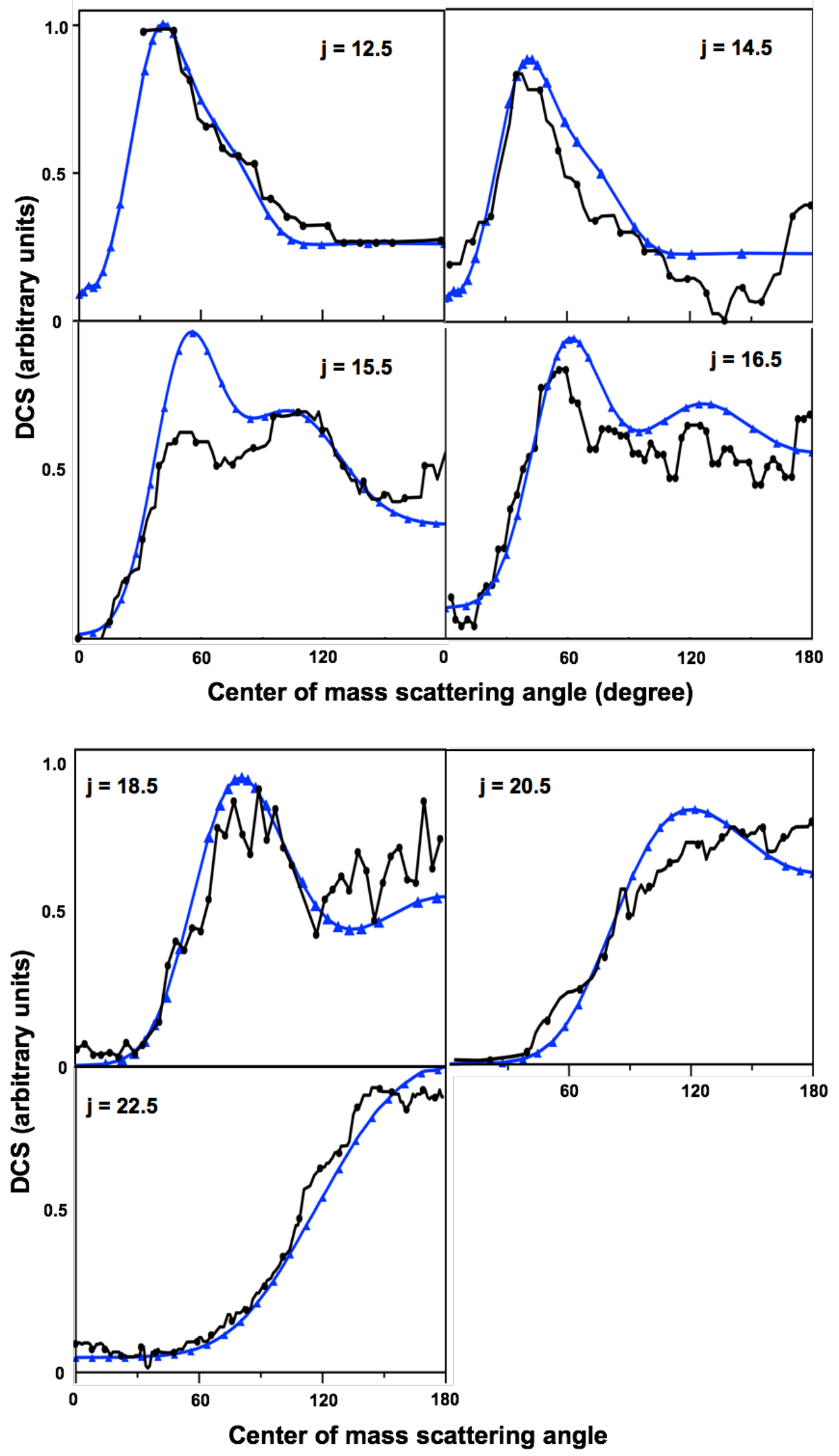
Figure 2.4 A comparison of the experimental and theoretical differential cross sections for the scattering of $\mathrm{NO}(v=0)$ with Ar for the indicated final rotational level. The black circles represent the experimental results and the blue triangles represent the theoretical predictions. 
molecule. These two peaks are more clearly resolved for $\mathrm{J}=15.5$, but the more forward peak is significantly reduced compared to theory. This may be due to interference from $\mathrm{J}=20.5$ which is also present in the image and enhances the more backscattered peak. We then investigate whether vibrational excitation of the NO molecule significantly affects the angular distributions of the rotationally inelastic scattering with Ar. The experimental differential cross section for a particular final rotational state in the $\mathrm{NO}(v=1) / \mathrm{Ar}$ system is compared to that in the $\mathrm{NO}(v=0) / \mathrm{Ar}$ system in Fig. 3.5 The signal-to-noise level of the $\mathrm{NO}(v=1) / \mathrm{Ar}$ system is lower than that in the $\mathrm{NO}(v=0) / \mathrm{Ar}$ system as may be seen. Nevertheless, the differential cross sections still show a rotational rainbow peak shifting to larger scattering angle for $\mathrm{NO} v=1$. We also see that the position of the rainbow maxima for $\mathrm{NO} v=1$ at each final rotational state almost tracks that of $\mathrm{NO} v=0$. There is only one significant exception, that is $j=12.5$. At $j=12.5$, a modest enhancement of the backward scattering distribution is obtained in the $\mathrm{NO}(v=1) / \mathrm{Ar}$ system which we do not observe for the $\mathrm{NO}(v=0) /$ Ar system nor in the theoretical DCS for $\mathrm{NO}(v=0)$. Aside from this level, the comparison of $v=1$ to $v=0$ does not reveal significant differences in the scattering distributions within the present signal-to-noise. The results obtained might not be that surprising since we only excite NO to $v=1$, and the equilibrium bond length is only $\sim 1 \%$ larger. Houston and coworkers observed only a small change in the angle of the rainbow maxima in the scattering of vibrationally excited $\mathrm{NO}(v=5)$ with $\mathrm{Ar}$ compared to the scattering of $\mathrm{NO}$ in the vibrational ground state with Ar. However, the root-mean-square spread in the $\mathrm{N}-\mathrm{O}$ distance is $\sqrt{3}$ times larger for $v=1$, suggesting an effect might be observable. It may be that by averaging over the $20 \mathrm{~K}$ distribution we 
obscure small changes in the DCS. Future studies that employ spectroscopic preparation of vibrationally excited levels while maintaining a rotationally very cold beam may have an improved chance of revealing subtle effects.
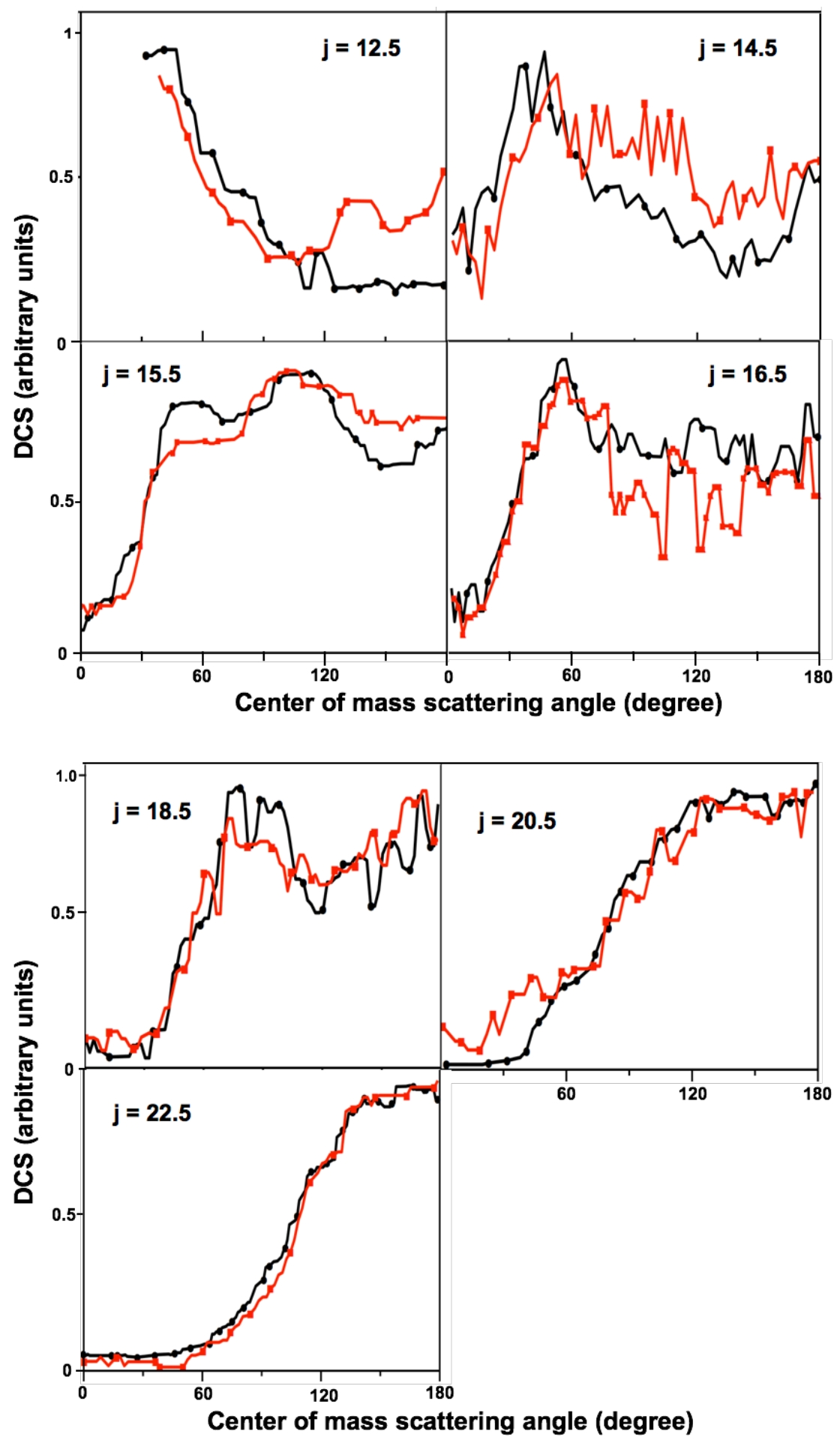
Figure 3.5 Comparisons of the experimental differential cross section distributions for the inelastic scattering of $\mathrm{NO}(v=0) / \operatorname{Ar}$ (black curve) and $\mathrm{NO}(v=1) / \mathrm{Ar}$ (red curve). Top panel compares $v=1$ to $v=0$ for $\mathrm{j}=12.5,14.5,15.5,16.5$, and down panel compares $v=1$ to $v=0$ for $\mathrm{j}=18.5,20.5$, and 22.5.

\subsection{CONCLUSION}

We have used crossed molecular beams coupled to dc slice velocity map imaging to investigate the effect of vibrational excitation on the inelastic scattering for $\mathrm{NO}$ with Ar. The inelastic collision process with collision energy of $3.0 \mathrm{kcal} \mathrm{mol}^{-1}$ for vibrationally excited $\mathrm{NO}$ at $v=1$ with Ar is examined and compared to that of the vibrational ground state $\mathrm{NO}$ with Ar. We observed the expected gradual shift of the rotational rainbow maxima to larger scattering angle as we probe higher rotational levels. Within the signal-to-noise, we did not observe significant scattering differences between the $\mathrm{NO}(v=1) / \mathrm{Ar}$ and $\mathrm{NO}(v=0)$.

\section{Acknowledgements}

This work was supported by the Director, Office of Science, Office of Basic Energy Science, Division of Chemical Science, Geoscience and Bioscience of the U.S. Department of Energy under Contract No. DE-SC0017130. DP acknowledges support from the Dutch National Science Foundation NWO-CW TOP project number 715.013.002. AGS gratefully acknowledges a Radboud Excellence Professorship for development of this collaboration. 


\section{CHAPTER 4}

Intersystem crossing in the exit channel

Hongwei Li, Alexander Kamasah, Arthur G. Suits*

Department of Chemistry, University of Missouri, Columbia, MO 65211, USA 


\begin{abstract}
Intersystem crossing (ISC), the non-radiative transition between two states with different multiplicity, plays an important role in photophysics, photochemistry, and photobiology. ISC is generally understood to be efficient when heavy atoms are present due to the strong spin-orbit coupling, or when strongly bound long-lived complexes are formed that increase the chance of finding the singlet/triplet intersection seam. Here we present evidence for a different ISC mechanism in the bimolecular reaction of $\mathrm{O}\left({ }^{3} \mathrm{P}\right)$ with the alkylamines. In crossed-beam experiments, the product velocity-flux maps are measured for aminoalkyl radicals produced from $\mathrm{H}$-abstraction on the methyl group giving $\mathrm{OH}$ radicals as co-fragments. Low product translational energy release and isotropic angular distributions indicate that such reactions undergo complex formation first before producing $\mathrm{OH}$ and aminoalkyl products. However, there is no well on the triplet potential energy surface that could support such a complex. Ab initio calculations suggest instead that ISC occurs in the exit channel region due to the long-range dipole-dipole interaction between the nascent radical product pair coupled with the vanishing singlet-triplet splitting at long range. ISC then leads to a deep hydroxylamine well before $\mathrm{OH}$ elimination.
\end{abstract}




\subsection{INTRODUCTION}

Intersystem crossing (ISC), the non-radiative transition between two electronic states with different spin multiplicity, is ubiquitous and important in fields ranging from chemical physics to chemical biology. ISC is involved in a wide range of applications including materials science $^{68}$, molecular photonics $^{69}$, photosensitizers ${ }^{70}$, and photodynamic therapy for cancer ${ }^{71}$. It is well studied that the "heavy-atom effect" promotes ISC since the inclusion of heavy atoms in the molecular structure enhances the spin-orbit coupling between singlet and triplet states. ${ }^{72-73}$ ISC is also observed in oxygen atom reactions with unsaturated hydrocarbons, in which case the strongly bound addition complex is long-lived, increasing the probability for the system to the singlet/triplet seam of intersection where ISC occurs. ${ }^{74-79}$ Here, we show that, even in the absence of heavy atoms or a strongly bound adduct on the initial triplet potential surface, ISC occurs in the course of bimolecular reactions of $\mathrm{O}\left({ }^{3} \mathrm{P}\right)$ with amines. In this case, we propose that ISC is promoted by the near-degeneracy of the singlet and triplet potential surfaces in the exit channel, along with the long-range dipole-dipole interaction and high dimensionality of the system that permits a brief period of recollision, leading finally to the deep hydroxylamine well on the singlet surface.

Amines, prototypical nitrogen-containing compounds, are found in the conventional fuels, such as coal, and renewable energy sources, such as biomass and biofuels. ${ }^{80-81}$ Burning of these nitrogen-containing compounds in the fuel is a principal source of nitrogen oxides $\left(\mathrm{NO}_{\mathrm{x}}\right)$ emitted to the atmosphere. ${ }^{80-82} \mathrm{NO}_{\mathrm{x}}$ represents $\mathrm{NO}, \mathrm{NO}_{2}$, and $\mathrm{N}_{2} \mathrm{O}$ that are harmful to the atmosphere, involved in formation of photochemical smog and acid rain, and $\mathrm{N}_{2} \mathrm{O}$ is also an effective greenhouse gas. ${ }^{80-82}$ The combustion chemistry of 
nitrogen compounds has been explored for decades, focusing on kinetic rate measurements, nitrogen conversion mechanism, and intermediates formed during the reaction and their roles in the overall process. ${ }^{80-83-84-81-82}$ However, a clear understanding of the reaction dynamics for nitrogen compounds, especially in the early step of the combustion process, is limited but desired to assist the successful development of accurate models for real combustion chemical systems.

Here, we investigated the mechanism of the elementary reactions in the initial combustion process, reaction of $\mathrm{O}\left({ }^{3} \mathrm{P}\right)$ radical with amines, dimethylamine (DMA) and trimethylamine (TMA). We have characterized the translational energy release and angular distributions during the reactions using crossed beam scattering combined with universal DC slice imaging as described previously ${ }^{85}$ and in Supporting Information (SI). Ab initio calculations on the energies and structures along the reaction pathways were also performed to gain insight to the underlying dynamics. Combining these experimental and theoretical studies suggests that ISC from triplet to singlet potential energy surfaces plays an important role in the bimolecular reaction dynamics for $\mathrm{O}\left({ }^{3} \mathrm{P}\right)$ reaction with amines.

\subsection{EXPERIMENTAL}

The electronic ground state atomic oxygen, $\mathrm{O}\left({ }^{3} \mathrm{P}\right)$, was generated from the photolysis of $\mathrm{SO}_{2}$ by $193 \mathrm{~nm}$ radiation. Amines (DMA or TMA) seeded in helium were crossed with the $\mathrm{O}\left({ }^{3} \mathrm{P}\right)$ beam at $90^{\circ}$ under single-collision conditions. The scattered products from the bimolecular reaction were ionized at the interaction region by an $\mathrm{F}_{2}$ excimer laser $(157 \mathrm{~nm}, 7.9 \mathrm{eV})$. The ions were then accelerated onto a position-sensitive detector that is gated to select a specific $\mathrm{m} / \mathrm{z}$ ratio. The resultant ion images were recorded 
using a charge-coupled device camera, employed with a high resolution real-time ion counting method using our megapixel acquisition program NuACQ. ${ }^{86}$ The sliced and centroided images were accumulated, reflecting the product velocity-flux contour maps with the speed and angular information for the reaction. 


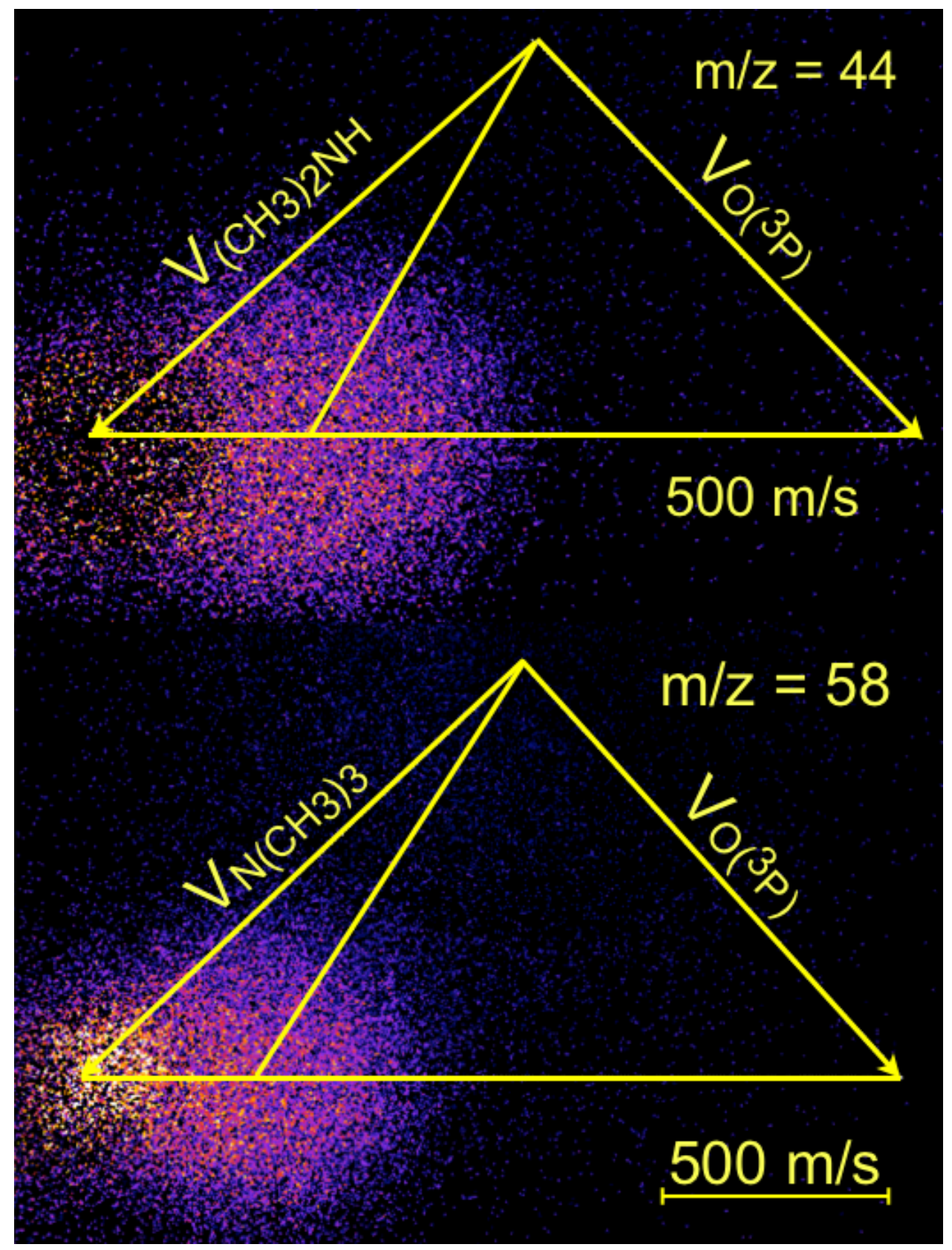


Figure 4.1 DC slice images top Dimethylamine (DMA) and bottom Trimethylamine (TMA) with Newton diagrams superimposed, with $\mathrm{O}\left({ }^{3} \mathrm{P}\right)$ after background subtraction and density-flux-correction at collision energies of 7.8 and $8.0 \mathrm{kcal} \mathrm{mol}^{-1}$, respectively. 


\subsection{RESULTS AND DISCUSSION}

For the bimolecular reaction of $\mathrm{O}\left({ }^{3} \mathrm{P}\right)$ with TMA at a collision energy of $\mathrm{E}_{\text {coll }}=$ $8.0 \mathrm{kcal} \mathrm{mol}^{-1}$, we detected only one product channel $\mathrm{m} / \mathrm{z} 58$ under our experimental conditions. This mass channel represents $\left(\mathrm{CH}_{3}\right)_{2} \mathrm{NCH}_{2}$ radical formed with hydroxyl radical $(\mathrm{OH})$ co-fragment after TMA is attacked by the $\mathrm{O}\left({ }^{3} \mathrm{P}\right)$ atom. The corresponding product scattering image after background subtraction and density-to-flux correction is shown in Figure 4.1 with a Newton diagram superimposed. The scattered radical products are examined in three distinct center-of-mass (c.o.m) angular ranges defined with respect to the TMA beam direction: forward $\left(\mathrm{FW}, 0-60^{\circ}\right)$, sideways ( $\left.\mathrm{SW}, 60-120^{\circ}\right)$ and backward (BW, $120-180^{\circ}$ ). Although the FW component is obscured by a strong photochemical background, the SW and BW components clearly reveal the underlying dynamics. The c.o.m. translational energy release distributions $P\left(E_{T}\right)$ of both $\mathrm{SW}$ and $\mathrm{BW}$ components peak at low energy, $\sim 10 \%$ of the collision energy (bottom panel in Figure 4.2). The c.o.m. angular distribution $T(\theta)$ (middle panel in Figure 4.2), including both SW and BW contributions, is flat, consistent with the isotropic image observed. These experimental results clearly indicate an indirect reaction mechanism. Formation of a long-lived adduct in a bimolecular reaction results in product translational energy distributions that peak at low energy because of randomization of the internal energy over the vibrational degrees of freedom in the complex. Additionally, if the complex lifetime is much longer than its rotational period, the system loses reference to the initial approach direction, giving rise 

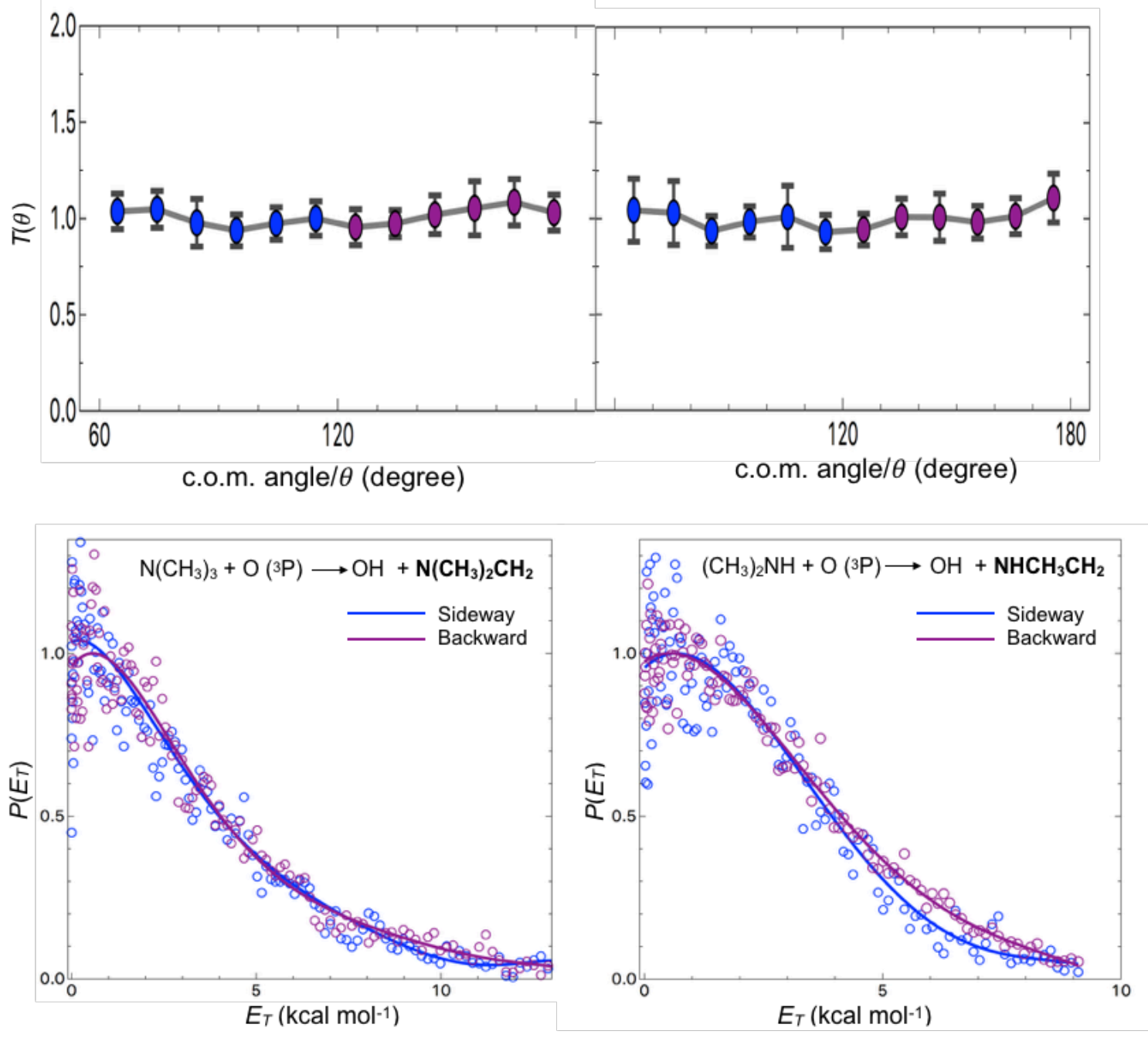
Figure 4.2 Center-of-mass angular $\mathrm{T}(\theta)$ (top) and translational energy $\mathrm{P}\left(\mathrm{E}_{\mathrm{T}}\right)$ (bottom) distributions for the reactions of $\mathrm{O}\left({ }^{3} \mathrm{P}\right)$ with TMA (left) and DMA (right) at collision energies of 8.0 and $7.8 \mathrm{kcal}^{\mathrm{mol}}{ }^{-1}$, respectively. The sideways $\left(60-120^{\circ}\right)$ component is in blue, and backward in purple $\left(120-180^{\circ}\right)$ The $\mathrm{P}\left(\mathrm{E}_{\mathrm{T}}\right)$ are fitted by least squares polynomial regressions. The $\mathrm{T}(\theta)$ are shown averaged every $10^{\circ}$ with error bars $( \pm \sigma)$ estimated by mean absolute deviation of the raw data in the corresponding angle range. 
to a symmetric scattering in the FW and BW directions and identical translational energy distributions in all directions. For complicated polyatomic systems in which the scattering is non-planar, this symmetric scattering becomes fully isotropic. ${ }^{27}$ Therefore, the low translational energy release and isotropic angular distributions of the scattered products indicate the importance of the complex-elimination mechanism in bimolecular reaction of $\mathrm{O}\left({ }^{3} \mathrm{P}\right)$ atom with TMA.

The reaction of $\mathrm{O}\left({ }^{3} \mathrm{P}\right)$ with DMA was also explored at collision energy of 7.8 kcal mol ${ }^{-1}$ A single product at $\mathrm{m} / \mathrm{z} 44$ was detected under our experimental conditions (shown in Figure 4.1). This indicates an $\mathrm{H}$ abstraction pathway producing $\mathrm{C}_{2} \mathrm{H}_{6} \mathrm{~N}$ radical in this reaction. There are two isomers for the $\mathrm{C}_{2} \mathrm{H}_{6} \mathrm{~N}$ radical, $\mathrm{CH}_{3} \mathrm{NHCH}_{2}$ arising from $\mathrm{H}$ abstraction at the methyl site, and $\mathrm{N}\left(\mathrm{CH}_{3}\right)_{2}$ produced by abstraction at nitrogen. Ab initio calculations performed using the CBS-QB3 method ${ }^{87}$ implemented in the Gaussian09 quantum chemistry software package ${ }^{88}$ were used to determine ionization energies (IEs) for these product radicals. The calculations predict that $\mathrm{CH}_{3} \mathrm{NHCH}_{2}$ radical has a vertical IE of $6.7 \mathrm{eV}$, easily accessed with our detection limit of $7.9 \mathrm{eV}$, whereas $\mathrm{N}\left(\mathrm{CH}_{3}\right)_{2}$ radical has a much higher vertical IE $(9.5 \mathrm{eV})$. Therefore, only $\mathrm{CH}_{3} \mathrm{NHCH}_{2}$ radical product is detected under our experimental conditions, even though the $\mathrm{N}\left(\mathrm{CH}_{3}\right)_{2}$ radical is also a possible product from this reaction. This inference was also proved by checking the reaction of $\mathrm{O}\left({ }^{3} \mathrm{P}\right)$ with the partially deuterated isotopologue $\left(\mathrm{CD}_{3}\right)_{2} \mathrm{NH}$ (see $\left.\mathrm{SI}\right)$. Only the $\mathrm{CD}_{3} \mathrm{NHCD}_{2}$ radical product was observed by our $7.9 \mathrm{eV}$ probe, consistent with the ionization energy calculations. This indicates the H/D removal by $\mathrm{O}\left({ }^{3} \mathrm{P}\right)$ radical we detected occurs at the methyl site rather than the amine site. Similar low translational energy release and isotropic angular distributions were also obtained here for the 


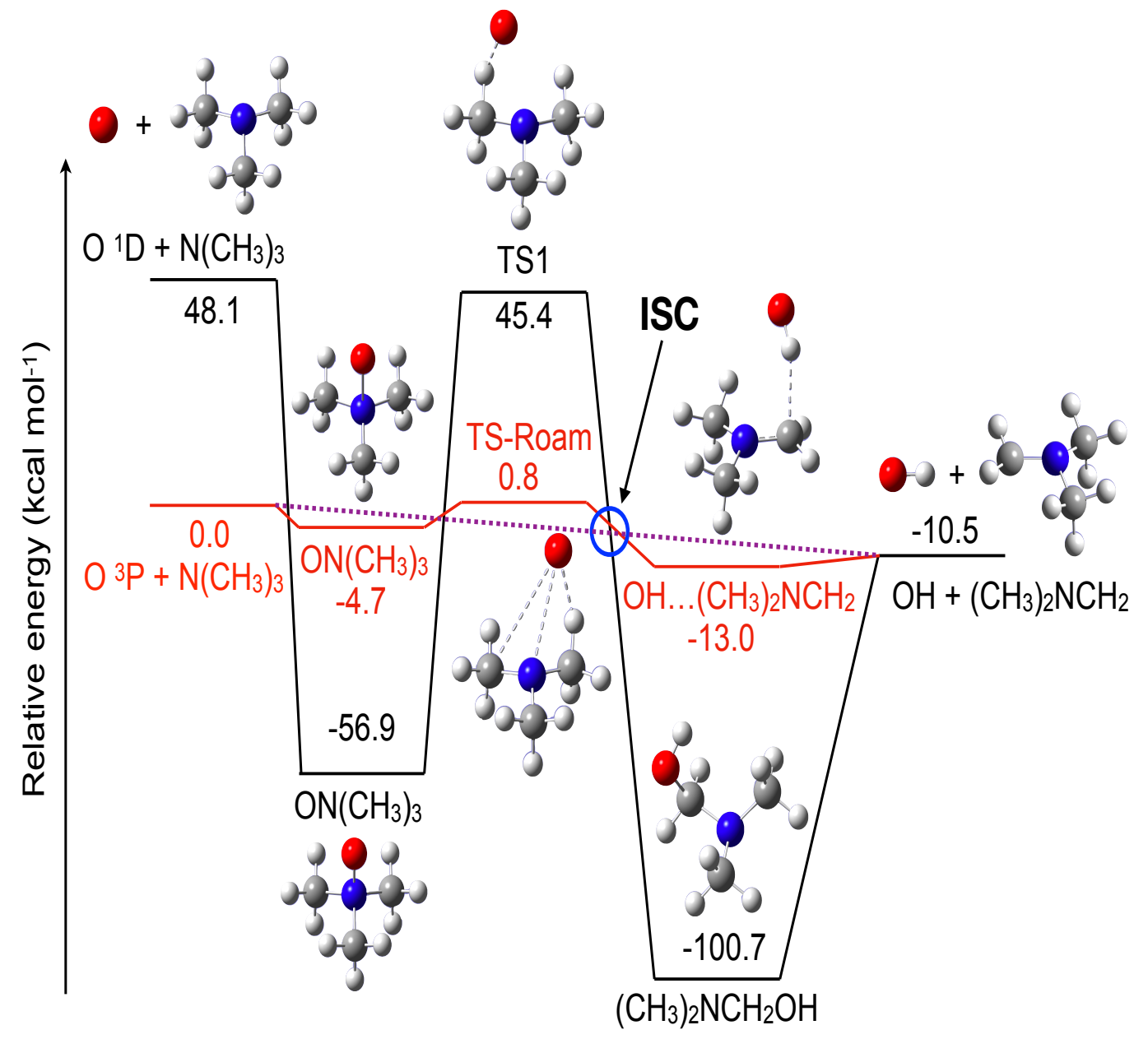


Figure 4.3. Key points on the triplet (red) and singlet (black) PESs of the $\mathrm{O}\left({ }^{3} \mathrm{P}\right)+$ TMA reaction. The diagram has been calculated at the CBS-QB3 level of theory. Relative energies are shown in $\mathrm{kcal} \mathrm{mol}^{-1}$. The purple dashed line illustrates the barrierless direct $\mathrm{H}$ abstraction pathway on the triplet surface with ISC occurrence indicated by a blue circle. 
deuterated (in SI) and non-deuterated (in Figure 4.6) systems, confirming again that the complex-elimination mechanism plays an important role in bimolecular reactions of $\mathrm{O}$ $\left({ }^{3} \mathrm{P}\right)$ with amines to produce $\mathrm{OH}$. The complex-elimination mechanism observed here agrees well with the previous cross-jet reactor study and kinetic measurements performed decades ago for the title reactions. ${ }^{89-90}$

The crucial underlying question now is the pathway for $\mathrm{OH}$ elimination from the complex. A careful search along the triplet and singlet potential energy surfaces (PESs) was performed by ab initio calculations at the CBS-QB3 level of theory. For the triplet PESs of the $\mathrm{O}\left({ }^{3} \mathrm{P}\right)+$ TMA reaction as shown in Figure 4.3, we could not locate any transition state (TS) for the direct $\mathrm{H}$ abstraction pathway to generate $\mathrm{OH}$ and $\left(\mathrm{CH}_{3}\right)_{2} \mathrm{NCH}_{2}$ products that we probed experimentally. This suggests that the direct reaction is barrierless. Interestingly, we find a roaming-type TS that has one low imaginary frequency $\left(200 \mathrm{~cm}^{-1}\right)$ indicating a flat PES in this region, and two very low bound frequencies (40 and $50 \mathrm{~cm}^{-1}$ ) corresponding to motions of the $\mathrm{O}$ atom relative to the TMA fragment. This roaming-type TS connects two shallow van der Waals complex wells, $\mathrm{O} \ldots \mathrm{N}\left(\mathrm{CH}_{3}\right)_{3}$ and $\left(\mathrm{CH}_{3}\right)_{2} \mathrm{NCH}_{2} \ldots \mathrm{OH}$ in the reaction pathway. However, based on the fact of the observed long-lived complex formation and relatively high collision energy $E_{\text {coll }}=8.0 \mathrm{kcal} \mathrm{mol}^{-1}$ in the experiment, this shallow well is not likely a key aspect of the reaction. We then searched through the singlet PESs since the coupling between singlet and triplet PESs inducing ISC is ubiquitous in chemistry and it is widely observed in $\mathrm{O}\left({ }^{3} \mathrm{P}\right)$ reactions with unsaturated hydrocarbons, such as $\mathrm{C}_{2} \mathrm{H}_{4}$ and $\mathrm{CH}_{2} \mathrm{CCH}_{2} \cdot{ }^{91-75-77} \mathrm{As}$ shown in the singlet PESs for the $\mathrm{O}\left({ }^{3} \mathrm{P}\right)+$ TMA reaction in Figure 4.3, one TS (TS1) and two very deep complex wells $\left(\mathrm{ON}\left(\mathrm{CH}_{3}\right)_{3}\right.$ and $\left.\left(\mathrm{CH}_{3}\right)_{2} \mathrm{NCH}_{2} \mathrm{OH}\right)$ are located on the 
reaction pathway to form the probed products. However, TS1 found here has a very high barrier energy, $45 \mathrm{kcal} \mathrm{mol}^{-1}$ above the $\mathrm{O}\left({ }^{3} \mathrm{P}\right)+$ TMA reactant asymptotic limit. Given the experimental collision energy of $8.0 \mathrm{kcal} \mathrm{mol}^{-1}$, the system does not have enough energy to surmount this barrier to form the detected products if the reaction accesses the singlet $\mathrm{ON}\left(\mathrm{CH}_{3}\right)_{3}$ entrance complex well via ISC. Therefore, the only plausible pathway for the reaction will be that the $\mathrm{O}\left({ }^{3} \mathrm{P}\right)$ radical attacks TMA to initiate direct $\mathrm{H}$ abstraction from methyl group. Then, due to the long-range dipole-dipole interaction and the high dimensionality of the system, $\mathrm{OH}$ and $\left(\mathrm{CH}_{3}\right)_{2} \mathrm{NCH}_{2}$ radicals do not part immediately but undergo multiple collisions. At the region where both triplet and singlet PESs have similar energy (as indicated by the blue circle in Figure 4.3), the system hops from triplet to singlet surface and then falls into a very deep hydroxylamine well, forming $\left(\mathrm{CH}_{3}\right)_{2} \mathrm{NCH}_{2} \mathrm{OH} 100 \mathrm{kcal} \mathrm{mol}^{-1}$ lower in energy than the reactants. The system will stay in the $\left(\mathrm{CH}_{3}\right)_{2} \mathrm{NCH}_{2} \mathrm{OH}$ well for a time much longer than its rotational period of the complex, then eliminate $\mathrm{OH}$ radical. In this case, the reaction mechanism features ISC leading to long-lived complex formation rather than being caused by it.

The PES for the $\mathrm{O}\left({ }^{3} \mathrm{P}\right)+\mathrm{DMA}$ reaction calculated at same level of theory is analogous to the $\mathrm{O}\left({ }^{3} \mathrm{P}\right)+$ TMA system as shown in Figure 4.4. No TS is found for the direct $\mathrm{H}$ abstraction channel, but a roaming-type TS is located between two shallow van der Waals complex wells along the reaction pathway on the triplet surface. However, again the roaming pathway may not play a key role due to the high collision energy and the possibility of barrierless $\mathrm{H}$ abstraction. On the singlet surface, two deep complex wells are present on the pathway forming the detected products, but the TS barrier on this pathway again is too high $\left(46.9 \mathrm{kcal} \mathrm{mol}^{-1}\right)$ to surmount given the $7.8 \mathrm{kcal} \mathrm{mol}^{-1}$ 


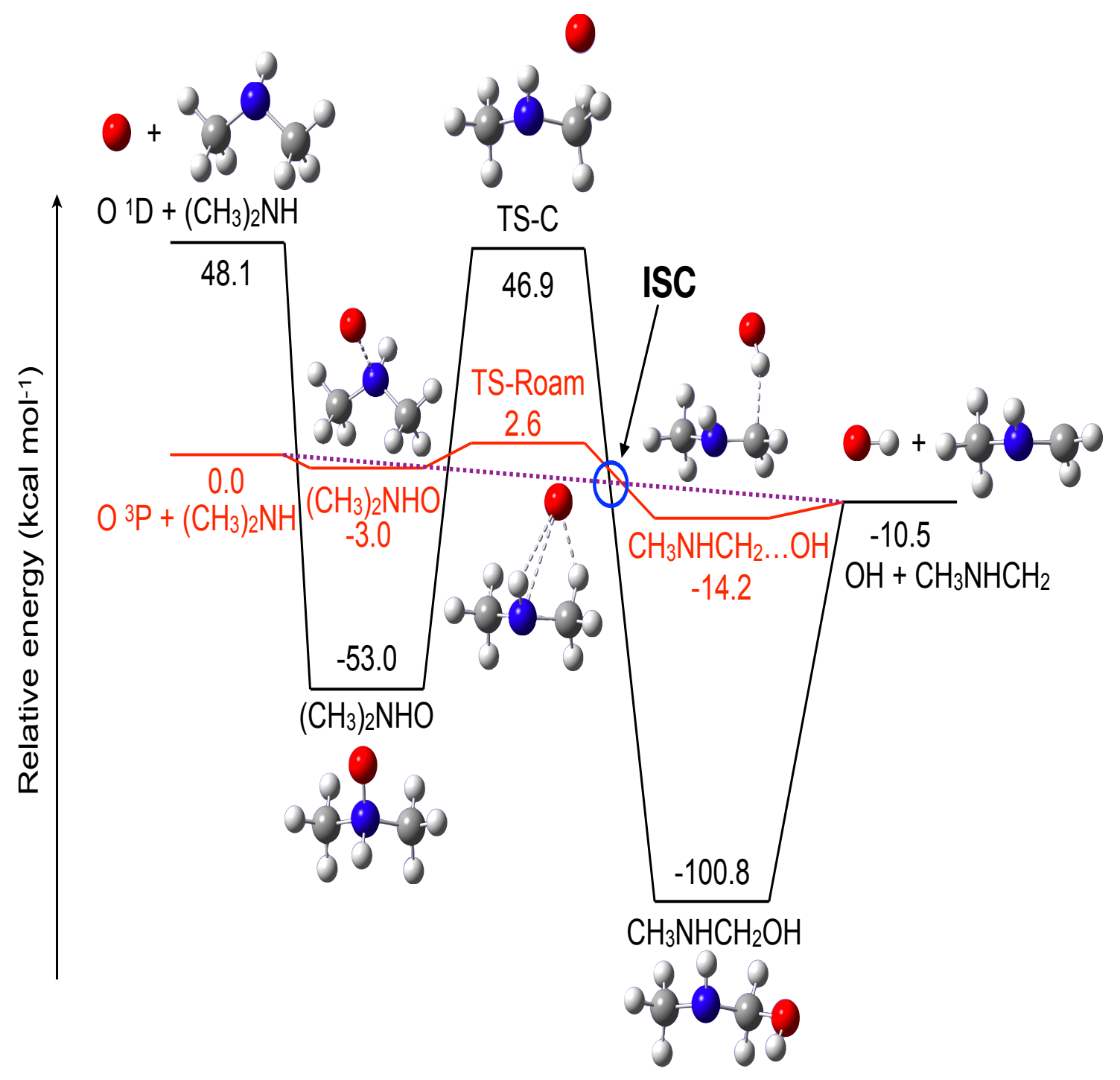


Figure 4.4. Key points on the triplet (red) and singlet (black) PESs of the $\mathrm{O}\left({ }^{3} \mathrm{P}\right)+$ DMA reaction, calculated at the CBS-QB3 level of theory. Relative energies are shown in $\mathrm{kcal} \mathrm{mol}^{-1}$. The purple dashed line illustrates the barrierless direct $\mathrm{H}$ abstraction pathway on the triplet surface with ISC occurrence indicated by a blue circle. 
experimental collision energy. Therefore, analogous to $\mathrm{O}\left({ }^{3} \mathrm{P}\right)+$ TMA reaction, the $\mathrm{O}\left({ }^{3} \mathrm{P}\right)$ + DMA reaction may proceed via direct $\mathrm{H}$-abstraction from a methyl group on DMA, followed by ISC to singlet surface leading to the very deep exit channel complex well $\left(\mathrm{CH}_{3} \mathrm{NHCH}_{2} \mathrm{OH}\right)$ before $\mathrm{OH}$ elimination to produce the detected radicals.

To understand the nature of ISC in the present case, we recall that the singlet and triplet surfaces are degenerate at long range for the pair of doublet radical products. In Figure 4.5, a representative structure that would follow the initial $\mathrm{H}$ abstraction is shown. Here, we calculate only $10 \mathrm{~cm}^{-1}$ energy splitting between the singlet and triplet states. If this initial abstraction leads to a period of recollision, as is commonly seen in polyatomic molecules, particularly given the high dimensionality of the system and the substantial dipole-dipole interaction in this case, the intersection seam of the singlet and triplet surfaces will be crossed many times. This permits ISC and access to the very deep well on the singlet surface. Although direct abstraction is possible on the triplet surface, the absence of a direct component in the experiment suggests that this reaction-mediated ISC is very efficient in this case. 


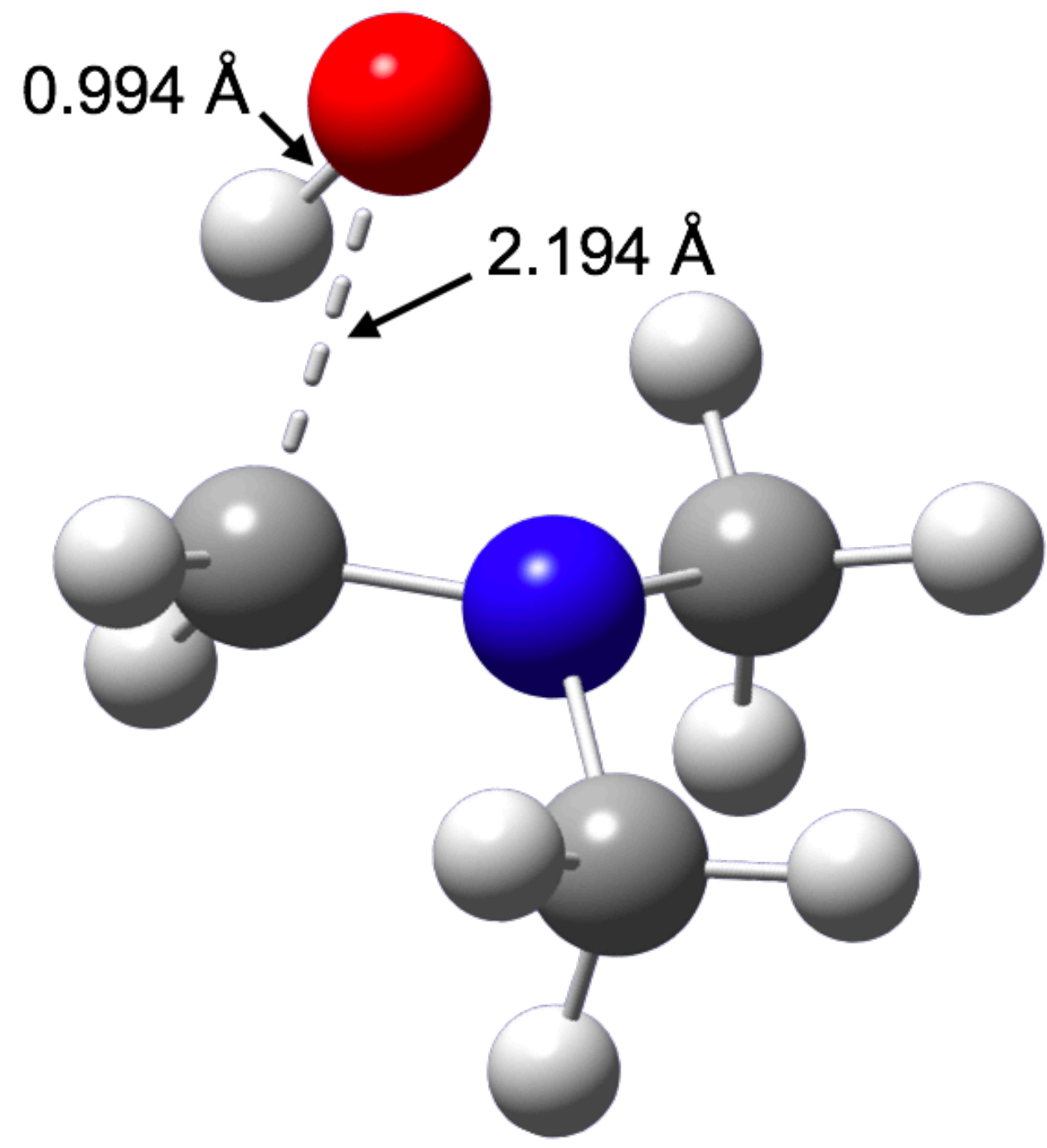

Figure 3.5 Geometric structure calculated at the wB97XD/6-311G(2d,d,p) level of theory. 
ISC in $\mathrm{O}\left({ }^{3} \mathrm{P}\right)$ reactions with unsaturated hydrocarbons has been well documented, and provides a clear contrast to what is seen here. ${ }^{74}$ Trajectory surface hopping calculations from Bowman and coworkers clearly revealed the mechanism in that case. ${ }^{91-}$

${ }^{75}$ For $\mathrm{O}\left({ }^{3} \mathrm{P}\right)+$ ethylene, there is a diradical addition complex bound by $25 \mathrm{kcal} \mathrm{mol}^{-1}$ that supports a long-lived complex. The bound diradical system hops to the nearby singlet potential surface where that configuration is a transition state connecting oxirane and acetaldehyde. Once on the singlet surface, a number of product channels are open to it. A key feature these two mechanisms have in common is presence of two radical sites that are weakly coupled: for $\mathrm{O}\left({ }^{3} \mathrm{P}\right)+$ ethylene it is within the $\cdot \mathrm{OCH}_{2} \mathrm{CH}_{2} \cdot$ diradical, while for $\mathrm{O}\left({ }^{3} \mathrm{P}\right)+\mathrm{TMA}$ it is the two radicals $\cdot \mathrm{OH}$ and $\cdot \mathrm{CH}_{2} \mathrm{~N}\left(\mathrm{CH}_{3}\right)_{2}$ interacting in the exit channel.

\subsection{CONCLUSION}

In conclusion, we have investigated the dynamics of the $\mathrm{H}$-abstraction process from the methyl group of amines (TMA and DMA) when reacting with ground state $\mathrm{O}\left({ }^{3} \mathrm{P}\right)$. The characterization of the low product translational energy release and isotropic angular distributions indicates that such reactions undergo complex formation first before producing $\mathrm{OH}$ and aminoalkyl products. Ab initio calculations indicate that the reaction is initiated by direct $\mathrm{H}$ abstraction from $\mathrm{O}$ attack, and then proceeds via ISC to the singlet surface forming a long-lived complex before $\mathrm{OH}$ elimination. This mechanism is not exclusive to amine reactions, and similar dynamics may occur in a large range of related systems yet to be studied. 


\section{Acknowledgments}

This work was supported by the Director, Office of Science, Office of Basic Energy

Science, Division of Chemical Science, Geoscience and Bioscience of the U.S.

Department of Energy under Contract No.DE-SC0017130. We are grateful to Prof. J.M.

Bowman for comments on the manuscript. 


\section{CHAPTER 5}

Imaging $\mathrm{H}$ abstraction dynamics in crossed molecular beams: $\mathrm{O}\left({ }^{3} \mathrm{P}\right)+$ propanol isomers

Hongwei Li, Alexander Kamasah, Arthur G. Suits*

Department of Chemistry, University of Missouri Columbia, MO 65211, United States 


\begin{abstract}
The crossed beam reaction dynamics of ground state $\mathrm{O}\left({ }^{3} \mathrm{P}\right)$ atoms with propanol isomers (1-propanol and 2-propanol) have been studied for the first time using the velocity map imaging technique. The hydroxypropyl radical products, generated from $\mathrm{H}$-abstraction of the secondary and tertiary $\mathrm{C}-\mathrm{H}$ groups of propanol isomers, were detected via single photon ionization at $157 \mathrm{~nm}$ under single collision conditions with collision energies of 8 and $10 \mathrm{kcal} \mathrm{mol}^{-1}$. Direct rebound dynamics were reflected by the angular distributions, that show sideways-backward scattering but more favorable backward scattering for both reactions under all collision energies detected here. All the translational energy distributions are peaking at low energy region, on average $20 \%-30 \%$ of the total available energy, indicating high internal excitation in the products. A "vertical" $\mathrm{H}$ abstraction mechanism was used here to address the internal excitation of the hydroxypropyl radicals.
\end{abstract}




\subsection{INTRODUCTION}

There has been a great amount of interest in reactions involving ground state oxygen atoms with hydrocarbons due to the very important roles they play in combustion ${ }^{92-93}$ and atmospheric chemistry. ${ }^{94} \mathrm{~A}$ very important mechanism that these reactions undergo is the hydrogen abstraction of which a lot of work has been reported. Luntz and Anderson ${ }^{95}$ coupled LIF to crossed molecular beams to probe $\mathrm{OH}$ rotational state from a series of saturated hydrocarbons. They reported that the vibrational state distribution of the nascent $\mathrm{OH}$ depends on what type of the hydrogen is abstracted but the final rotational distribution of $\mathrm{OH}$ was the same for all types of hydrocarbons. Similar work by ${ }^{96}$, this time involving unsaturated hydrocarbon with $\mathrm{O}\left({ }^{3} \mathrm{P}\right)$ also confirmed the importance of hydrogen abstraction in the reaction mechanism. In their work, they observed very small rotational excitation of the nascent $\mathrm{OH}$. Suits and coworkers ${ }^{97}$ carried out imaging studies of $\mathrm{O}\left({ }^{3} \mathrm{P}\right)$ with a series of saturated hydrocarbons by obtaining differential cross sections and translational energy and observed a backward sideways scattering angular distribution of the alkyl products with low average translational energy release. A further study by Suits and coworkers ${ }^{98}$ involving pentane and $\mathrm{O}\left({ }^{3} \mathrm{P}\right)$ and $\mathrm{O}$ $\left({ }^{1} \mathrm{D}\right)$ revealed very contrasting dynamics, whiles backwards scattering was observed for

$\mathrm{O}\left({ }^{3} \mathrm{P}\right)$, they observed isotropic and forward scattering for the $\mathrm{O}\left({ }^{1} \mathrm{D}\right)$ reaction. Lee and his group employed crossed molecular beam with photoionization mass spectrometry to study the reaction dynamics of $\mathrm{O}\left({ }^{3} \mathrm{P}\right)$ with benzene and its deuterated analogs by measuring the velocity distribution and the angular distribution of the reaction products. They concluded in their report that, oxygen addition and hydrogen elimination were the 
major reaction pathways for this reaction. The Casavecchia group ${ }^{99}$ also carried out both experimental and theoretical calculation of $\mathrm{O}\left({ }^{3} \mathrm{P}\right)$ with propene by combining crossedmolecular beam with mass spectrometric detection and reported the importance of intersystem crossing in such class of systems which accounted for $20 \%$ of the product yield. Although the dynamics of $\mathrm{RH}$ reactions with $\mathrm{O}\left({ }^{3} \mathrm{P}\right)$ have been studied extensively, that cannot be said for the oxygenated hydrocarbons especially alcohols (ROH). Over the past few decades, there has been great interest in alcohols for combustion processes ${ }^{100-103}$ as an alternative form of fuel because of its environmentally friendly nature. Although the kinetics and rate of these reactions are very well documented ${ }^{104-107}$, there has been limited dynamics study for these reactions. Most of the reaction dynamics of these systems focused on reactions with $\mathrm{Cl}, \mathrm{F}$ and $\mathrm{OH}$ radicals. Osman and his group ${ }^{108}$ carried out an ab initio quantum chemical studies of $\mathrm{H}$ abstraction from 2-propanol by $\mathrm{OH}$ radical and reported that collision-controlled mechanism may play a significant role in the $\mathrm{H} \propto$ abstraction of the reaction. Tully and his group ${ }^{109}$ determined the absolute rate coefficient for the reaction between methanol and $\mathrm{OH}$ radical by obtaining branching ratio for the reaction, their results showed that at low temperatures $\mathrm{H}$ abstraction from the methyl dominates and at high temperature $\mathrm{H}$ abstraction is favored on the hydroxyl hydrogen. Taatjes and coworkers ${ }^{110}$ used time-resolved infrared absorption spectroscopy to study the reaction between $\mathrm{Cl}$ atoms with ethanol and its deuterated forms by measuring the absolute rate coefficient, in their work, they reported that the reaction proceeds predominately by $\mathrm{H} \propto$ and the co-fragment $\mathrm{HCl}$ produced was vibrationally excited. Recently our group studied the dynamics of $\mathrm{F}$ atom with 1-propanol by probing the alkoxy radical. The results showed the backward translational energy peaking at a higher 
energy than the sideways and a forward component was observed for the center of mass angular distribution ${ }^{111}$.

Suits and coworkers have carried out detailed dynamic studies of $\mathrm{Cl}$ atom reactions with a series of alcohol by using slice imaging. In one of their work, they combined crossed molecular beams with velocity map imaging to to probe the alkoxy radicals of methanol, ethanol, and isopropanol reactions with $\mathrm{Cl}\left(2 \mathrm{P}^{3} / 2\right)$. Their results revealed that about $30-40 \%$ of the available energy of the reaction was channeled into product translation and the center of mass angular distribution showed a sideways and backwards distribution, an indication of a rebound mechanism ${ }^{112}$. A recent work by Estillore et. al ${ }^{113}$ also from the group studied the interaction of chlorine radicals with butanol and its isomers at low and high collision energy. The results revealed backward scattering distribution with respect to the alcohol beam at low collision energy and the scattering distribution shifts to forward direction with increase in collision energy. The results also revealed that about $80 \%$ of the collision energy was seen in the forward scattered product for high collision energy. The Zare group ${ }^{114}$ performed state-to-state dynamics of $\mathrm{Cl}$ reactions with methanol at $5.6 \mathrm{kcal} / \mathrm{mol}$ collision energy by looking at the rovibrational state distribution of the nascent $\mathrm{HCl}$. They concluded in their work that about $84 \%$ of the product $\mathrm{HCl}$ was formed in the $\mathrm{v}=0$ with the remaining $16 \%$ in $\mathrm{v}=1$ state. The angular distribution of the products $\mathrm{HCl}(\mathrm{v}=1)$ showed forward scattering with very little internal energy of $\mathrm{CH}_{2} \mathrm{OH}$, whiles significant amount of internal energy was observed for $\mathrm{v}=0$ an indication of stripping mechanism for $\mathrm{v}=1$. Quantum state differential cross sections of the reaction between chlorine atoms and methanol was studied by Murray and coworkers ${ }^{115}$ by imaging $\mathrm{HCl}$ at different rotational levels in their 
vibrational ground state. They observed both forward and backward scattering distribution. At lower J, scattering was in the forward component and shifts to backwards scattering for higher $\mathrm{J}$.

To our best of knowledge, very limited or no dynamic studies work has been carried out on $\mathrm{O}\left({ }^{3} \mathrm{P}\right)$ reactions with alcohol. The only dynamic studies that has been reported so far involve $\mathrm{O}\left({ }^{1} \mathrm{D}\right)$ reactions with methanol and ethanol and its isotopologue by Goldstein and Wiesenfeld ${ }^{116}$ were they used LIF to probed the vibrational distributions and product ratios of $\mathrm{OH}$ and $\mathrm{OD}$. They reported that the insertion/elimination was the main mechanism by which $\mathrm{O}\left({ }^{1} \mathrm{D}\right)$ attacks the alcohol and the attacks occurs at the $\mathrm{O}-\mathrm{H}$ bond, although some may occur at the $\mathrm{C}-\mathrm{H}$ bond. Based on the low vibrational excitation of the $\mathrm{OH}(\mathrm{OD})$, they concluded that the abstraction process was a minor process. Here we present a preliminary reaction dynamics study of $\mathrm{H}$-abstraction reactions of $\mathrm{O}$ $\left({ }^{3} \mathrm{P}\right)$ with 1-propanol, 2-propanol using our crossed-molecular beam dc slice imaging. The product hydroxyalkyl radicals were probed via single photon ionization at $157 \mathrm{~nm}$ and their images recorded and analyzed after density-flux correction and background subtraction.

\subsection{EXPERIMENTAL DETAILS}

The scattering experiments were carried out in a crossed molecular beam apparatus combined with a DC slice ion imaging, which has been previously described elsewhere ${ }^{26-117}$. The apparatus consists of a reaction chamber and two source chambers that are perpendicular to each other. Both molecular beams were produced in a separate supersonic expansion in the two source chambers $\left(\sim 10^{-7}\right.$ torr base pressure and $\sim 10^{-5}$ 
torr operational pressure) and skimmed into the reaction chamber. Photolysis of $\mathrm{SO}_{2}$ by a $193 \mathrm{~nm}$ excimer laser was used to generate $\mathrm{O}\left({ }^{3} \mathrm{P}\right)$ atom and it is known that this does not produce any O $\left({ }^{1} \mathrm{D}\right) .{ }^{118-119}$ A $5 \% \mathrm{SO}_{2}(\geq 99.9 \%$, Sigma-Aldrich) seeded in He was pulsed from a piezoelectric stack valve $\mathrm{e}^{37}$ with a $120 \mu \mathrm{m}$ translational actuator and $50 \mu$ s pulse duration. $1 \mathrm{~mm}$ diameter capillary tube was mounted on the exit of the nozzle plate of the stack valve, and $193 \mathrm{~nm}$ radiation (from a GAM ArF excimer laser) was loosely focused onto the capillary to photolyze $\mathrm{SO}_{2}$ to generate a very intense $\mathrm{O}\left({ }^{3} \mathrm{P}\right)$ beam. Another piezoelectric disc valve was used to pulse 1-propanol or 2-propanol ( $\geq 99.9 \%$, SigmaAldrich) molecular target beam. By seeding them 5\% in $\mathrm{He}$ or $\mathrm{H}_{2}$, the collision energy was changed from $8 \mathrm{kcal} \mathrm{mol}^{-1}$ to $10 \mathrm{kcal} \mathrm{mol}^{-1}$. The scattered hydroxyalkyl radical products were ionized with a $\mathrm{F}_{2}$ excimer laser $(157 \mathrm{~nm}, 7.9 \mathrm{eV})$ as soon as the product exits the interaction region. The ions were then accelerated via a four-electrode dc slice ion optics assembly to impact on a dual microchannel plate (MCP) detector coupled to a fast phosphor screen ${ }^{7}$. The detector was gated for the center slice of the scattered product ions and the resultant images were recorded using a charge-coupled device (CCD) camera and analyzed with our data acquisition program. Background subtraction and density-to-flux corrections were performed prior to transforming the scattering distributions in the center-of mass coordinate. 


\subsection{RESULTS}

Crossed-molecular beam scattering with single photon ionization for many years has shown to be a sensitive technique to investigate bimolecular reactions. In probing the hydroxyalkyl radical using our $157 \mathrm{~nm}$ excimer laser, we need to take into account the detection efficiency for various radical products from the particular hydrogen abstraction sites. Both propanol isomers have two distinct abstraction sites: one at the hydroxyl end of the molecule and the other involving primary, secondary, and tertiary $\mathrm{C}-\mathrm{H}$ groups.
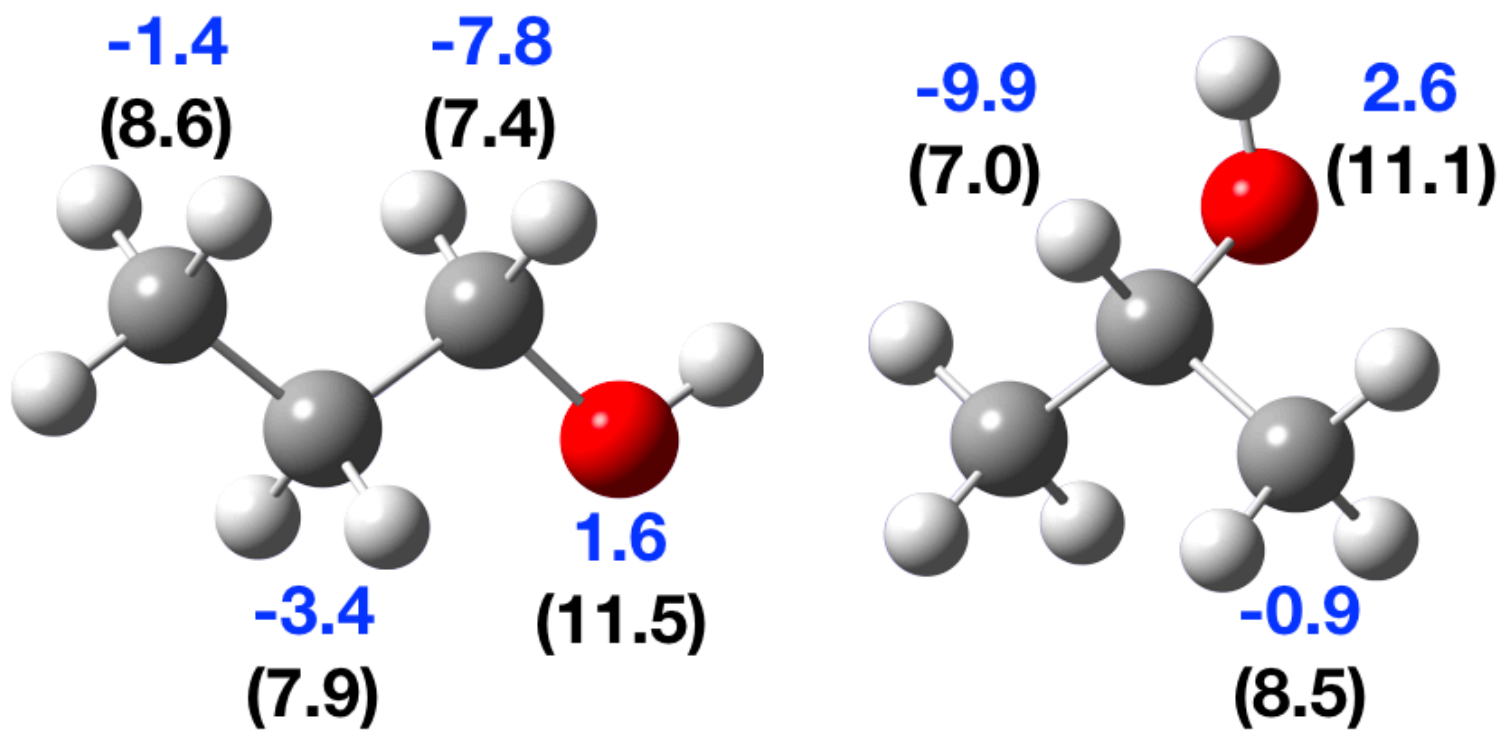

Figure.5.1 Lowest energy structures of 1-propanol (left) and 2-propanol (right). Reaction enthalpies at $0 \mathrm{~K}$ (numbers in blue color, $\mathrm{kcal} \mathrm{mol}^{1}$ ) at different abstraction sites are calculated at CBS-QB3 level of theory. Numbers in parenthesis are vertical ionization energies (eV) of corresponding product radicals. 
Figure 5.1 presents the reaction enthalpies $(0 \mathrm{~K})$ for $\mathrm{H}$ abstraction by $\mathrm{O}\left({ }^{3} \mathrm{P}\right)$ at the indicated sites, and the vertical ionization energies $(\mathrm{eV})$ of corresponding product radicals (in parentheses).

The stationary point geometries for propanol isomers and their $\mathrm{H}$ abstraction radical products are optimized and their energies are calculated at the CBS-QB3 level of theory. Even though $\mathrm{H}$ abstractions from both hydroxyl group and $\mathrm{C}-\mathrm{H}$ groups are energetically accessible according to the reaction enthalpies, the vertical ionization energy calculations indicate selectivity on the radical products that we can detect with our single photon ionization $(7.9 \mathrm{eV})$. For 1-propanol, the ionization energy of the $\mathrm{H}$ abstraction product from the $\mathrm{OH}$ group and primary $\mathrm{C}-\mathrm{H}$ group is higher than our detection probe of $7.9 \mathrm{eV}$, while the abstraction product from the secondary $\mathrm{C}-\mathrm{H}$ group is lower. Thus, the radical products of the $\mathrm{H}$ abstraction on the $\alpha$ - and $\beta-\mathrm{H}$ sites are detected in the present work for 1-propanol. Analogously, for 2-propanol, we only detect the $\beta$-H abstraction radical product that is on the tertiary $\mathrm{C}-\mathrm{H}$ group. 


\section{$\mathbf{O}\left({ }^{3} \mathbf{P}\right)+1$-propanol}
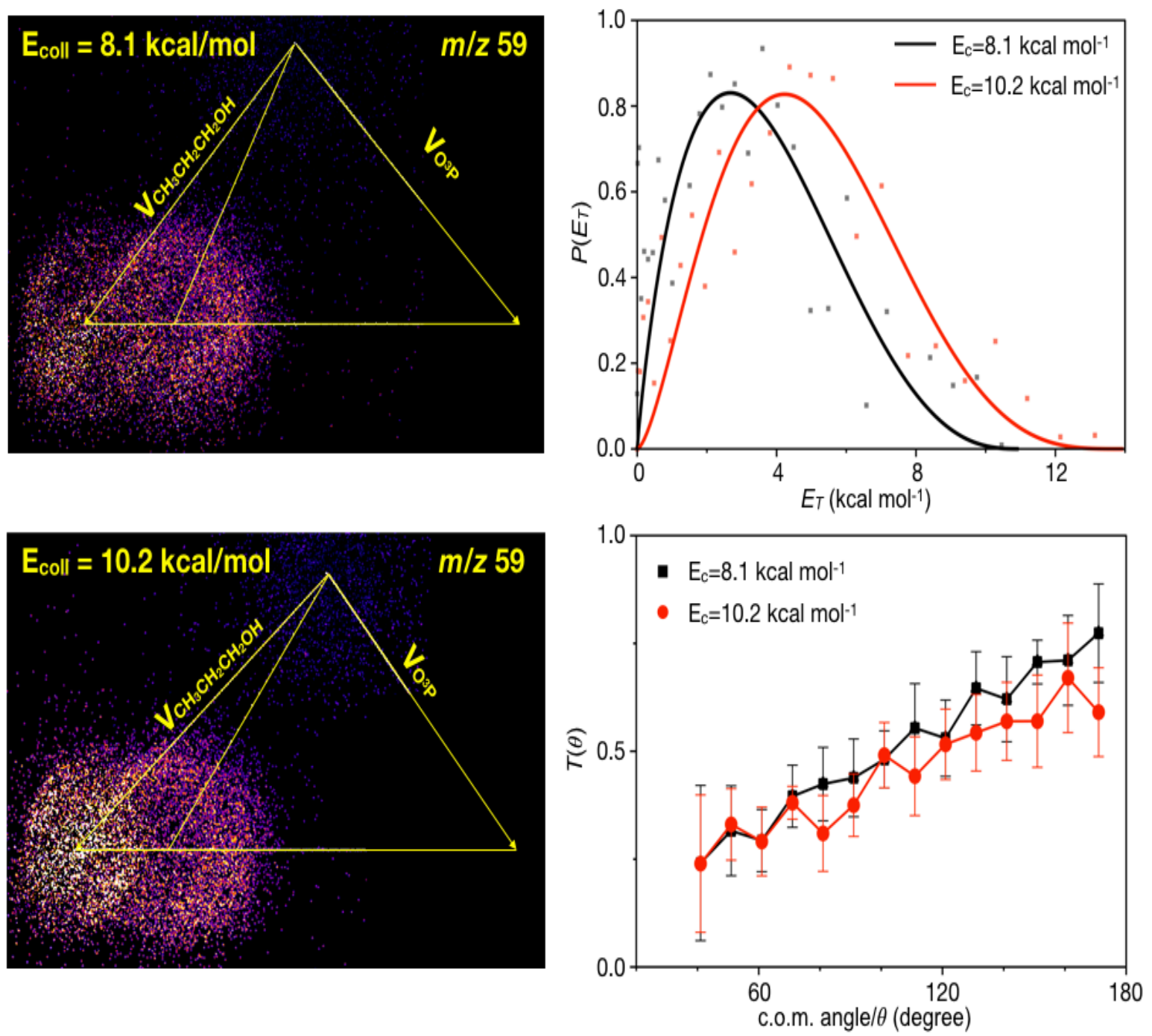

Figure 5.5. Sliced scattering images for the reaction of $O\left(\beta^{3}\right)$ ) with 1-propanol (Newton diagrams superimposed on them) under collision energy of 8.1(left top panel) and 10.2 (left bottom panel) $\mathrm{kcal} \mathrm{mol}^{1}$, and their corresponding global translational energy release $\left(P\left(E_{T}\right)\right.$, right top panel) and center-of-mass angular $(T(\theta)$, right bottom panel) distributions. The $T(\theta)$ distributions are shown averaged very $10^{\circ}$ with error bars $( \pm \sigma)$ estimated by mean absolute deviation of the raw data in the corresponding angle range. 
The reactive scattering images of the 1-hydroxypropyl radical products from the reaction of $\mathrm{O}\left({ }^{3} \mathrm{P}\right)$ with 1-Propanol at collision energies $\left(\mathrm{E}_{\mathrm{c}}\right)$ of 8.1 and $10.2 \mathrm{kcal} \mathrm{mol}^{-1}$ (with the Newton diagrams superimposed on them) are shown in the left panels of Figure 5.2. The 1-hydroxypropyl radical that we are probing here are produced from the $\mathrm{H}$ abstraction from the $\alpha$ - and $\beta-\mathrm{H}$ sites of 1-propanol as we mentioned early. We performed background subtraction by recording images with the $193 \mathrm{~nm}$ photolysis laser off and the $157 \mathrm{~nm}$ probe laser on to isolate the reactive scattering signals from the radicals produced by the photodissociation of 1-propanol at $157 \mathrm{~nm}$. Unfortunately, the intense photochemical signal creates substantial noise and brings uncertainty in the reactive flux of the forward component. For this reason, we omitted the first $45^{\circ}$ of the forward component in the further analysis for this $\mathrm{O}\left({ }^{3} \mathrm{P}\right)+1$-propanol reaction. The right panels of Figure 2 show the corresponding global translational kinetic energy and centerof-mass angular distributions at two different collision energies $\left(E_{c}=8.1 \mathrm{kcal} \mathrm{mol}^{-1}\right.$ in black and $\mathrm{E}_{\mathrm{c}}=10.2 \mathrm{kcal} \mathrm{mol}^{-1}$ in red). The average translational energy released at the collision energy of $10.2 \mathrm{kcal} \mathrm{mol}^{-1}$ and $8.1 \mathrm{kcal} \mathrm{mol}^{-1}$ is 5.3 and $3.5 \mathrm{kcal} \mathrm{mol}^{-1}$, respectively. And the angular distributions at both collision energies show both sideway and backward scattering but preferential backward with respect to the direction of the propanol beam. 


\section{$\mathbf{O}\left({ }^{3} \mathbf{P}\right)+2$-propanol}
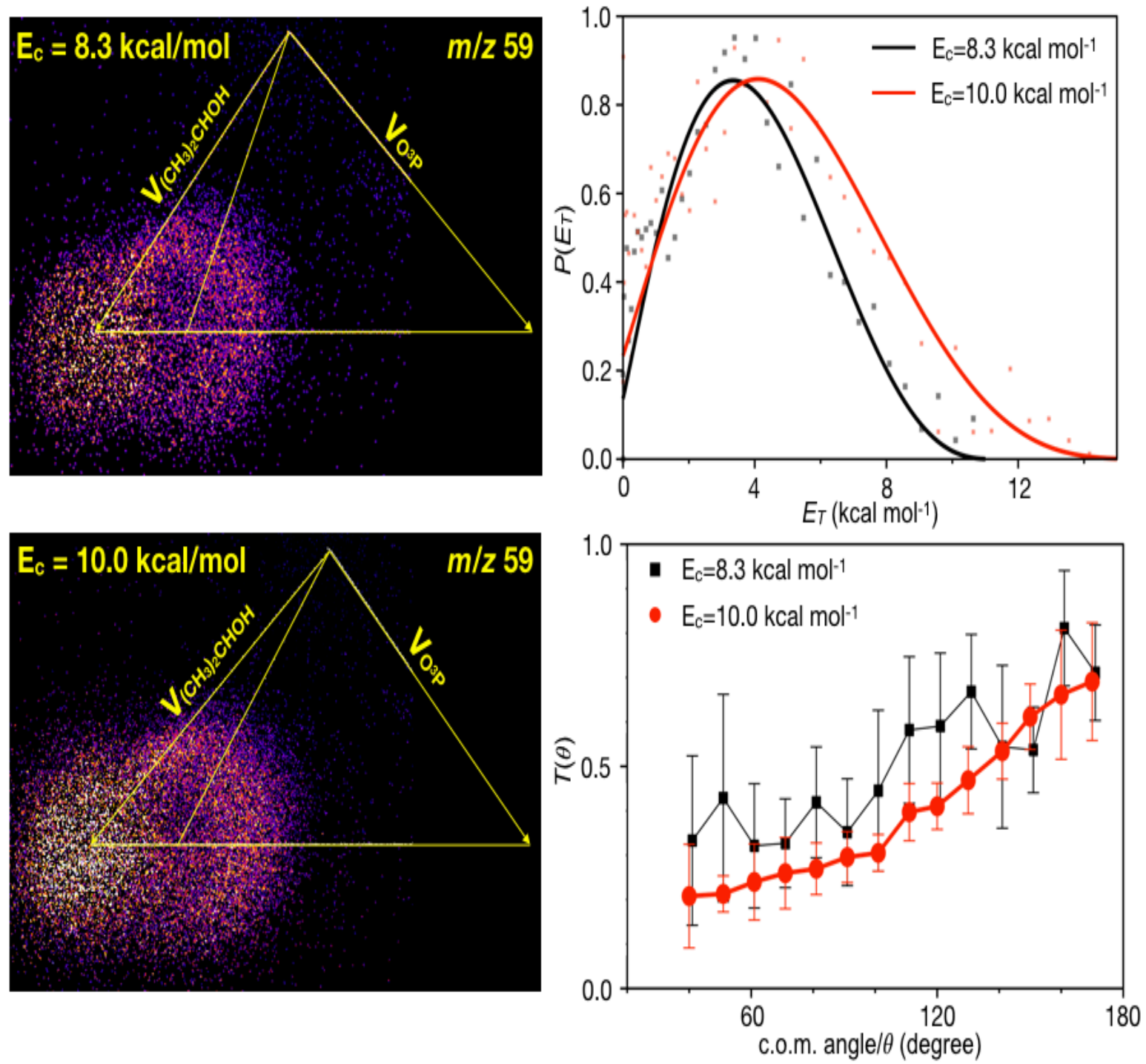

Figure 5.6. Sliced scattering images for the reaction of $O(\beta)$ ) with 2-propanol under collision energy of 8.3 (left top panel) and 10.0 (left bottom panel) $\mathrm{kcal} \mathrm{mol}^{-1}$, and their corresponding global translational energy release $\left(P\left(E_{T}\right)\right.$, right top panel) and center-of-mass angular $(T(\theta)$, right bottom panel) distributions. The $T(\theta)$ distributions are shown averaged very $10^{\circ}$ with error bars $( \pm \sigma)$ estimated by mean absolute deviation of the raw data in the corresponding angle range. 
The left panels of Figure 5.3 show the sliced reactive scattering images of 2hydroxypropyl radical products for the $\mathrm{O}\left({ }^{3} \mathrm{P}\right)+2$-propanol reaction at collision energy of $8.3 \mathrm{kcal} \mathrm{mol}^{-1}$ (top) and $10.0 \mathrm{kcal} \mathrm{mol}^{-1}$ (bottom). For this reaction, the 2-hydroxypropyl radical products that we probed here are only stemming from the $\mathrm{H}$-abstraction from $\alpha-\mathrm{H}$ sites of 2-propanol according to the ionization calculations. The total available energy for this reaction is 18.2 and $19.9 \mathrm{kcal} \mathrm{mol}^{-1}$ for collision energy of 8.3 and $10.0 \mathrm{kcal} \mathrm{mol}^{-1}$, respectively. Analogously to 1-propanol reaction, due to the large background interference in the forward direction, we have omitted the first $45^{\circ}$ component in the further analysis for this $\mathrm{O}\left({ }^{3} \mathrm{P}\right)+2$-propanol reaction. The global translational energy release distributions are shown in the right top panel of Figure 5.3, with average translational energy of 4.2 and $5.4 \mathrm{kcal} \mathrm{mol}^{-1}$ for the collision energy of 8.3 and $10.0 \mathrm{kcal}$ mol $^{-1}$ accounting for $23 \%$ and $27 \%$ of the total available energy, respectively. The centerof-mass angular distributions of both collision energies are shown in the right bottom panel of Figure 5.3, indicating again more preferential backward scattering with respect to the propanol beam direction.

\subsection{DISCUSSION}

The reaction of ground state atomic oxygen with 1-propanol and 2-propanol has been studied with kinetic methods and the reaction rates of the $\mathrm{H}$-abstraction from $\mathrm{C}-\mathrm{H}$ group were measured. ${ }^{120}$ The activation energy of both reactions was measure to be 12.5 and $9.1 \mathrm{~kJ} \mathrm{~mol}^{-1}$ for 1-propanol and 2-propanol, respectively. 2-propanol has lower activation energy due to the weaker bond of its tertiary C-H group. No special steric effects were suggested in the reactions of the atomic oxygen with both propanol alcohols 
from the kinetic measurement. The present work represents the further details of the underlying dynamics of the hydrogen abstraction for the oxygen atom $\mathrm{O}\left({ }^{3} \mathrm{P}\right)+$ propanol isomer reactions. The global differential cross sections as well as the translational energy partitioning were measured for the first time of these two reactions.

The angular distributions for both reactions under collision energies of 8 and 10 $\mathrm{kcal} \mathrm{mol}^{-1}$ show sideways-backward scattering and more favorable backward scattering is clearly observed, which implies direct rebound dynamics. Although we are not sensitive to the sharply forward-scattered products in the present work, we can rule out the forward-backward/isotropic symmetry that indicates the formation of complex during the reaction. This rebound dynamic involves small (but non-vanishing) impact parameter collisions, leading to direct reaction via a collinear transition state geometry, $\mathrm{C}-\mathrm{H}-\mathrm{O}$, as suggested by the well-established line-of-centers model. Here, we refer to the collinear C$\mathrm{H}-\mathrm{O}$ coordinate as the line of center, not the line between the center-of-mass of the reactants.

The product translational energy release is also measured that gives important information about the dynamics. For both reactions, we re-plot the translational energy release distributions of the sideway $\left(60^{\circ}-120^{\circ}\right)$ and backward $\left(120^{\circ}-180^{\circ}\right)$ components against the collision energy as the reduced translational energy distributions (shown in Figure 5.4). For the collision energy of both 8 and $10 \mathrm{kcal} \mathrm{mol}^{-1}$, the similar reduced translation energy distributions are presented in the $\mathrm{SW}$ and $\mathrm{BW}$ direction, indicating that the energy partitioning is not sensitive to the collision energy in the energy region of the present study. The detailed average translational energy of the SW/BW components and 
the fraction of total energy (and collision energy) appearing in translation are summarized in Table 1. In both $\mathrm{O}\left({ }^{3} \mathrm{P}\right)+$ propanol reactions, the average translational energy release
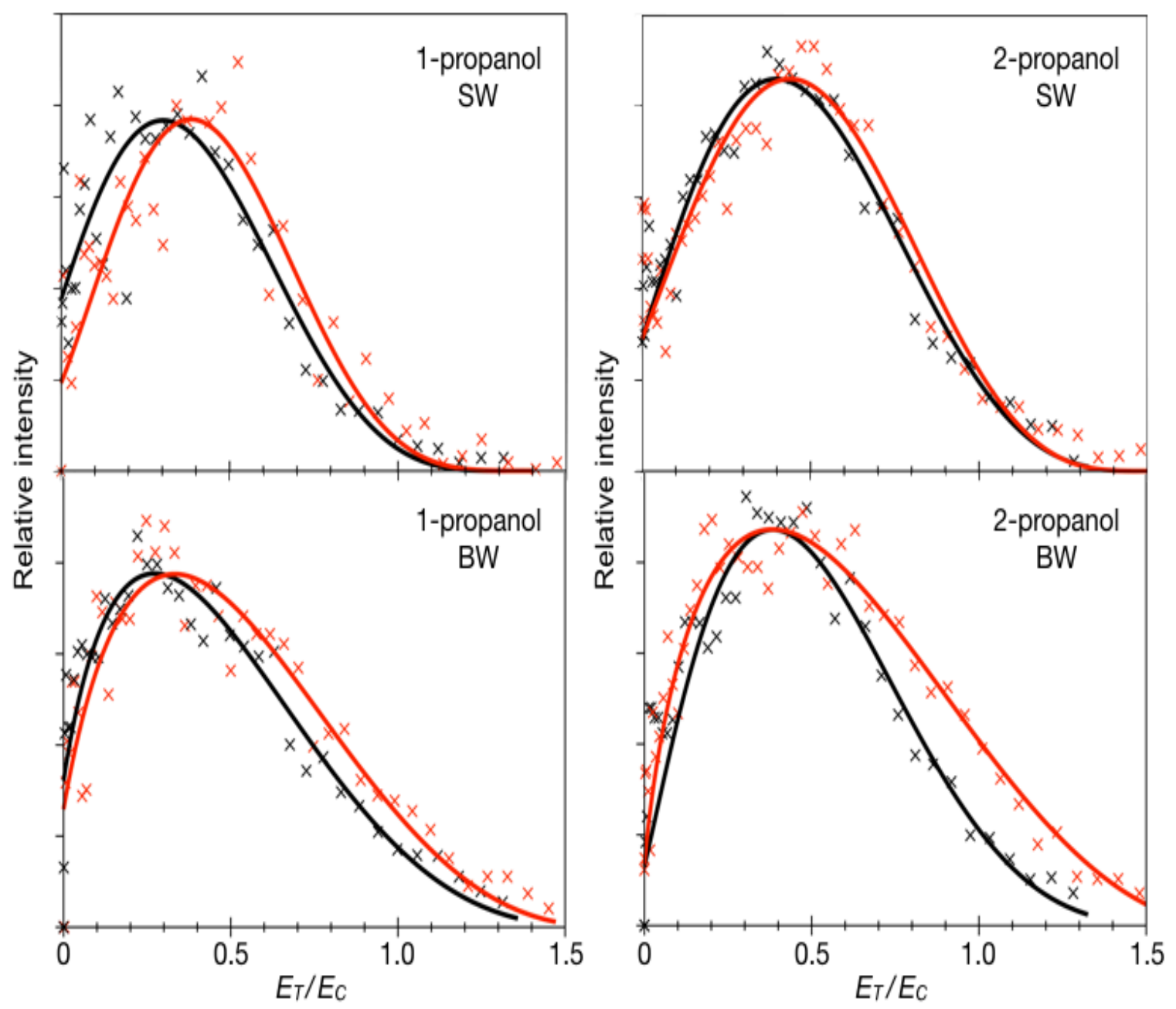

Figure 5.7. Reduced translational energy distributions for sideway (SW) and backward (BW) scattering regions of the hydroxyalkyl products for low collision ( $\left.8 \mathrm{kcal} \mathrm{mol}^{-1}\right)$ and high collision $\left(\sim 10 \mathrm{kcal} \mathrm{mol}^{-1}\right)$ energy. 
accounts for $20-30 \%$ of the total available energy and the energy partitioning increases with increasing collision energy. A simple way to view the dynamics of these reaction is an impulsive model that was widely used to understand photodissociation events.

Impulsive model assumes that the total available energy is released as an impulse along the breaking bond with momentum conservation between the two "atoms" governing the translational energy release. In this model, the fraction of translational energy is simply dependent upon the mass combination: $f_{T}=m_{A B C} m_{B} /\left(m_{A B} m_{B C}\right)$ for dissociation of $\mathrm{ABC}$, where $\mathrm{C}$ is bonded to (and recoils from) B, A remains initially as a spectator. ${ }^{121}$ This model predicts an $f_{T}$ of $54 \%$ of the total available energy for the $\mathrm{O}\left({ }^{3} \mathrm{P}\right)+$ propanol systems, which is too high compared with experiment. Furthermore, this model cannot explain the collision energy dependence. The pure impulsive picture fails here since it assumes that all the energy is available for the partitioning between the fragments, which is unlikely if there is some internal excitation in recoiling fragments (e.g. hydroxyalkyl radical product) that does not relax completely during the $\mathrm{OH}-\mathrm{C}$ bond breaking. Another simple kinematic model for the linear triatomic system suggested by Evans et al. can also be used to predict the average translational energy release: $\left\langle E_{T}\right\rangle=$ $E_{c} \cos ^{2} \beta+E_{R} \sin ^{2} \beta$, where $\beta$ is the skew angle for the reaction, $E_{c}$ is the collision energy, and $E_{R}$ is the reaction energy. The skew angle is defined for $\mathrm{A}+\mathrm{BC}$ by $\cos ^{2} \beta=$ $\left(m_{A} m_{B}\right) /\left(m_{A B} m_{B C}\right)$ and embodies key kinematics for the reaction. In this model, the remaining energy is allocated to vibrational excitation of the newly formed $\mathrm{OH}$ bond, therefore, it provides a rough limiting view to account for relaxation of the hydroxyalkyl 
radical from the TS geometry. The $\mathrm{OH}$ radical generated from the titled reaction could be vibrationally excited given the experimental collision energy. In the previous LIF study of the $\mathrm{O}\left({ }^{3} \mathrm{P}\right)+$ saturated alkane by Andresen and Luntz, $\mathrm{OH}$ radical was found to be rotationally cold regardless of the nature of the alkane reagent, but vibrational excitation of $\mathrm{OH}$ strongly depends on the type of hydrogen abstracted and increases dramatically across the series primary to secondary to tertiary. For the titled reactions, the skew angle is acute, as typically seen in heavy-light-heavy hydrogen transfer reactions, so the first term strongly dominates and predicts that the average translational energy is very close to the collision energy. This overestimate on the translational energy from this model suggests that the internal excitation in the hydroxyalkyl radical products plays an important role for the titled reactions.

A reasonable explanation for the internal excitation of the hydroxyalkyl radicals is the "vertical" abstraction mechanism that was invoked by Liu et al. in $\mathrm{O}+$ n-butane reaction and Whitney et al. in $\mathrm{F}+\mathrm{C}_{2} \mathrm{H}_{6}$ reaction. In this picture, the $\mathrm{C}-\mathrm{H}$ bond breaks so rapidly that hydroxypropyl radical cannot relax to its minimum energy geometry, resulting in a certain amount of energy "locked" in the $\mathrm{C}_{3} \mathrm{H}_{6} \mathrm{OH}$ moiety. This view is equivalent to the Franck-Condon picture that the hydrogen transfer is a "vertical" rather than an "adiabatic" process. Some available energy will be partitioned into vibrational excitation of the hydroxypropyl radical product in a non-statistical and mode-specific manner, associated with significant structural changes between propanol parent molecule and hydroxypropyl radical product. We have optimized the geometries of 1-propanol and 2-propanol and calculated the energy of hydroxypropyl moiety at this geometry at the CBS-QB3 level of theory. The minimum energy structures of the hydroxypropyl radical 
products for each reaction were also optimized and their energy was calculated at the same level of theory. The energy difference between the vertical and adiabatic radical products from the $\mathrm{H}$-abstraction is $8.2 \mathrm{kcal} \mathrm{mol}^{-1}$ for 2-propanol, and $6.0 \mathrm{kcal} \mathrm{mol}^{-1}$ or 7.3 $\mathrm{kcal} \mathrm{mol}^{-1}$ for 1-propanol with $\alpha-\mathrm{H}$ or $\beta$-H abstraction. The major geometry changes accounting for this difference are summarized in Table 2. For instance, the C-C-C angle changes from $\sim 113^{\circ}$ to $\sim 121^{\circ}$ in both 2-propanol and 1-propanol by $\alpha-\mathrm{H}$ abstraction. And the $\mathrm{H}-\mathrm{C}-\mathrm{C}-\mathrm{C}$ dihedral angle changes from $\sim 122^{\circ}$ to $\sim 165^{\circ}$ in 1-propanol by $\beta-\mathrm{H}$ abstraction. These significant geometric changes as listed in Table 2.0 result in the corresponding vibrational excitation in the hydroxypropyl radicals, which could account for $\sim 50 \%$ or more of the total available energy depending on the collision energy. We also expect that $\sim 20 \%$ of the total available energy is partitioned to rotational excitation of hydroxypropyl radical product. The translational energy release is reduced in the sideway direction as shown in Table 1.0, indicating greater internal energy in the larger impact parameter collisions. This is consistent with greater rotational excitation for the sideway scattered products.

Apart from the $\mathrm{O}+\mathrm{n}$-butane reaction, another previous $\mathrm{Cl}+$ alcohol reactions studied by Ahmed et al. observed similar dynamics ${ }^{112}$, i.e. dominant backward scattering and only $\sim 35 \%-40 \%$ of the total available energy partitioned to translation. We suspect a similar mechanism may account for the substantial internal excitation in the hydroxyalkyl product, although the details will need to be modified by the different relaxation energies and barriers, etc. With addition of present work, this "vertical" reaction mechanism seams to play an important role in $\mathrm{H}$-abstraction dynamics in polyatomic systems. 


\subsection{CONCLUSION}

We have performed an imaging study of the reaction dynamics of ground state atomic oxygen with propanol isomers under single collision conditions with well-defined collision energies of 8 and $10 \mathrm{kcal} \mathrm{mol}^{-1}$. The hydroxypropyl radical products, generated from the $\alpha-\mathrm{H}$ and $\beta-\mathrm{H}$ abstraction for 1-propanol and $\alpha-\mathrm{H}$ abstraction for 2-propanol, were detected with our universal VUV $(157 \mathrm{~nm})$ soft ionization probe with velocity map imaging technique. The global differential cross sections and translational energy distributions were obtained from these images. Backward-sideways scattering but more favorable backward scattering was observed for both propanol isomer reactions under two different collision energy in the experiment. Low average translational energy release was obtained, accounting for $20 \%-30 \%$ of the total available energy, which indicates that a large amount of energy is released as the internal excitation of the products. As seen in the $\mathrm{O}+\mathrm{n}$-butane reaction, this can be explained by a modification of the triatomic model called as "vertical" $\mathrm{H}$ abstraction mechanism. In this picture, the $\mathrm{H}$ atom transfer is treated as a prompt "vertical" transition process, so that the hydroxypropyl radical does not have enough time to relax from the parent propanol geometry to the minimum energy geometry. This will lead to high internal excitation of the hydroxypropyl radical that is also supported by the ab initio calculations. This "vertical" reaction mechanism may play an important role in $\mathrm{H}$-abstraction dynamics in more polyatomic systems. 
Table 1.0 Summary of results on the translational energy partitioning for $O\left({ }^{\beta} P\right)+$ propanol isomers

\begin{tabular}{|c|c|c|c|c|c|c|c|c|}
\hline & $E_{C^{a}}$ & $E_{a v l^{b}}$ & $\begin{array}{c}<E_{T}> \\
S W^{c}\end{array}$ & $\begin{array}{l}<E_{T}> \\
B W d\end{array}$ & $\begin{array}{c}<E_{T}>/ E c \\
S W e\end{array}$ & $\begin{array}{c}<\boldsymbol{E}_{T}>/ \boldsymbol{E}_{c} \\
B W\end{array}$ & $\begin{array}{c}<E_{T}>/ E_{a v l} \\
S W g\end{array}$ & $\begin{array}{c}<\boldsymbol{E}_{\boldsymbol{T}}>/ \boldsymbol{E}_{\boldsymbol{a} v} \\
B W h\end{array}$ \\
\hline \multirow{2}{*}{ 1-Propanol } & 8.1 & $\begin{array}{c}15.9 \\
11.5\end{array}$ & 3.25 & 3.81 & 0.41 & 0.48 & $\begin{array}{l}0.20^{1} \\
0.28\end{array}$ & $\begin{array}{l}0.24 i \\
0.33 j\end{array}$ \\
\hline & 10.2 & \begin{tabular}{|c}
18.0 \\
0.20
\end{tabular} & 4.75 & 5.3 & 0.46 & 0.51 & $\begin{array}{l}0.26 \\
0.35\end{array}$ & $\begin{array}{l}0.29 \\
0.39\end{array}$ \\
\hline \multirow[t]{2}{*}{ 2-Propanol } & 8.3 & 18.2 & 3.51 & 4.26 & 0.42 & 0.51 & 0.19 & 0.23 \\
\hline & 10.0 & 19.9 & 4.76 & 5.45 & 0.48 & 0.55 & 0.24 & 0.27 \\
\hline
\end{tabular}

${ }^{a}$ Collision energy. ${ }^{b}$ Total available energy (collision energy + reaction energy release). ${ }^{c}$ Average translational energy in the sideway direction. ${ }^{d}$ Average translational energy in the backward direction. ${ }^{e}$ Fraction of collision energy apprearing in translation of the sideway component. ${ }^{f}$ Fraction of collision energy apprearing in translation of the backward component. ${ }^{g}$ Fraction of total available energy apprearing in translation of the sideway component. ${ }^{h}$ Fraction of total available energy apprearing in translation of the backward component. ${ }^{i}$ Results from $\alpha$-H abstraction site of 1-propanol. ${ }^{j}$ Results from $\beta$-H abstraction site of 1-propanol. 
Table 2.0 Results of ab initio calculations performed as described in the text (hartrees, degrees, angstroms).

\begin{tabular}{|c|c|c|c|c|c|c|}
\hline & $\mathrm{CH}_{3} \mathrm{CH}_{2} \mathrm{CHOH}$ & $\mathrm{CH}_{3} \mathrm{CH}_{2} \mathrm{CHOH}$ & $\mathrm{CH}_{3} \mathrm{CH}_{2} \mathrm{CHOI}$ & $\mathrm{CH}_{3} \mathrm{CH}_{2} \mathrm{CHOH}$ & $\left(\mathrm{CH}_{3}\right)_{2} \mathrm{CHOH}$ & $\left(\mathrm{CH}_{3}\right)_{2} \mathrm{CHOH}$ \\
\hline Energy & -193.336314 & -193.345812 & -193.327093 & -193.338695 & -193.341587 & -193.354680 \\
\hline$\angle O C 1 C 2$ & $108.0^{\circ}$ & $114.5^{\circ}$ & - & - & - & - \\
\hline$\angle \mathrm{C}_{1 C 2} \mathrm{C}_{3}$ & - & - & $112.8^{\circ}$ & $121.4^{\circ}$ & $112.6^{\circ}$ & $121.4^{\circ}$ \\
\hline$\angle O C_{1} 1 C_{2} C 3$ & $180.0^{\circ}$ & $170.8^{\circ}$ & $180^{\circ}$ & $166.6^{\circ}$ & $120.3^{\circ}$ & $143.1^{\circ}$ \\
\hline$\angle \mathrm{HC1C2C3}$ & $59.0^{\circ}$ & $-41.9^{\circ}$ & $-122.4^{\circ}$ & $-165.3^{\circ}$ & - & - \\
\hline O-C1/0-C2 & 1.427 & 1.375 & - & - & 1.439 & 1.383 \\
\hline $\mathrm{C} 1-\mathrm{C} 2$ & 1.521 & 1.488 & 1.521 & 1.484 & 1.526 & 1.495 \\
\hline $\mathrm{C} 2-\mathrm{C} 3$ & - & - & 1.531 & 1.489 & 1.525 & 1.490 \\
\hline
\end{tabular}

${ }^{*}$ The radical structure at the geometry of its parent molecule. The lowest energy structure of the radical. Bond length of $\mathrm{O}-\mathrm{Cl}$ for $\mathrm{CH}_{3} \mathrm{CH}_{2} \mathrm{CHOH}$ and bond length of $\mathrm{O}-\mathrm{C} 2$ for $\left(\mathrm{CH}_{3}\right)_{2} \mathrm{COH}$.

\section{Acknowledgments}

This work was supported by the Director, Office of Science, Office of Basic Energy

Science, Division of Chemical Science, Geoscience and Bioscience of the U.S.

Department of Energy under Contract No.DE-SC0017130. 


\section{Bibliography}

1. Herschbach, D. R. Molecular dynamics of elementary chemical reactions. Science, (1987).

2. Lee, Y. T. Molecular beam studies of elementary chemical processes. Science, (1987).

3. Polanyi, J. C. Some concepts in reaction dynamics. Science, (1987).

4. Levine, R. D. Molecular reaction dynamics. Cambridge University Press, (2005).

5. Murray, C. and Orr-Ewing, A. J. The dynamics of chlorine-atom reactions with polyatomic organic molecules. Int. Rev. Phys. Chem., 23, 435-482 (2004).

6. Choi, J.-H. Radical-radical reaction dynamics: A combined crossed-beam and theoretical study. Int. Rev. Phys. Chem., 25, 613-653 (2006).

7. Townsend, D., Minitti, M. P. and Suits, A. G. Direct current slice imaging. Rev Sci Instrum, 74, 2530-2539 (2003).

8. Roger, C. Atomic and molecular beams. Springer, (2001).

9. Ramsey, N. F. Molecular beams. Calendon Press, (1956).

10. Bernstein, R. B. Chemical dynamics via molecular beam and laser techniques. Calendon Press, (1982).

11. Fluendy, M. A. D. L., K.P. Chemical applications of molecular beam scattering. Chapman and Hall, (1973).

12. Houston, P. L. . Chemical kinetics and reaction dynamics. McGraw-Hill Companies, Inc., (2001).

13. Pauly, H., Buck, H. Determination of intermolecular potentials by the inversion of molecular beam scattering data. II. High resolution measurements of differential 
scattering cross sections and the inversion of the data for $\mathrm{Na}-\mathrm{Hg}$. J Chem Phys, 54, 1929 (1971)

14. Brink, G. O. Electron bombardment molecular beam detector. Rev Sci Instrum, 37, 857-860 (1966).

15. Wessel, G. L., H. Hyperfine structures of silver and gold by the atomic beam magnetic resonance method. Phys Rev Lett, 92, (1953).

16. Datz, S. T., E.H. Atom-molecule reaction of hydrogen studied by molecular beams. Chemical Physics, 39, (1963).

17. Fite, W. L. B., R.T. Scattering of h atoms by d2 molecules. J Chem Phys, 24, 18. Yang, X. New low background crossed molecular beam apparatus: Low background detection of $\mathrm{H}_{2}$. Rev Sci Instrum, 69, (1998).

19. Yang, X., Lin, J., Lee, Y. T., Blank, D. A., Suits, A. G. and Wodtke, A. M. Universal crossed molecular beams apparatus with synchrotron photoionization mass spectrometric product detection. Rev Sci Instrum, 68, 3317-3326 (1997).

20. Balucani, N., Capozza, G., Leonori, F., Segoloni, E. and Casavecchia, P. Crossed molecular beam reactive scattering: From simple triatomic to multichannel polyatomic reactions. Int. Rev. Phys. Chem., 25, 109-163 (2006).

21. Capozza, G., Segoloni, E., Leonori, F., Volpi, G. G. and Casavecchia, P. Soft electron impact ionization in crossed molecular beam reactive scattering: The dynamics of the $\mathrm{O}\left({ }^{3} \mathrm{P}\right)+\mathrm{C}_{2} \mathrm{H}_{2}$ reaction. Journal of Chemical Physics, 120, 4557-4560 (2004). 22. Fu, B. N., Han, Y. C., Bowman, J. M., Leonori, F., Balucani, N., Angelucci, L., Occhiogrosso, A., Petrucci, R. and Casavecchia, P. Experimental and theoretical studies 
of the $\mathrm{O}\left({ }^{3} \mathrm{P}\right)+\mathrm{C}_{2} \mathrm{H}_{4}$ reaction dynamics: Collision energy dependence of branching ratios and extent of intersystem crossing. Journal of Chemical Physics, 137, (2012).

23. Davis, H. F. Photodissociation dynamics of gaseous $\mathrm{CPCO}(\mathrm{CO}) 2$ and ligand exchange reactions of $\mathrm{CPCOH}_{2}$ with $\mathrm{C}_{3} \mathrm{H}_{4}, \mathrm{C}_{3} \mathrm{H}_{6}$, and $\mathrm{NH}_{3}$. J Phys Chem A, 116, (2012).

24. Hinrichs, R. Z. and Davis, H. F. C-C versus $\mathrm{C}-\mathrm{H}$ bond activation of alkynes by early second-row transition metal atoms. Journal of Physical Chemistry, 112, 3010-3019 (2008).

25. Willis, P. A., Stauffer, H. U., Hinrichs, R. Z. and Davis, H. F. Rotatable source crossed molecular beams apparatus with pulsed ultraviolet vacuum ultraviolet photoionization detection. Rev Sci Instrum, 70, 2606-2614 (1999).

26. Joalland, B., Shi, Y., Estillore, A. D., Kamasah, A., Mebel, A. M. and Suits, A. G. Dynamics of chlorine atom reactions with hydrocarbons: Insights from imaging the radical product in crossed beams. Journal of Physical Chemistry A, 118, $9281-95$ (2014). 27. Joalland, B., Shi, Y., Kamasah, A., Suits, A. G. and Mebel, A. M. Roaming dynamics in radical addition-elimination reactions. Nat Commun, 5, 4064 (2014).

28. Shi, Y., Kamasah, A. and Suits, A. G. H abstraction channels in the crossed-beam reaction of F + 1-propanol, 1-butene and 1-hexene by dc slice imaging. J Phys Chem A, $120,8933-8940$ (2016).

29. Shi, Y. Y., Kamasah, A., Joalland, B. and Suits, A. G. Crossed-beam de slice imaging of fluorine atom reactions with linear alkanes. Journal of Chemical Physics, 142, (2015).

30. Feldman, D. R. Z., R.N. Mul tiphoton ionization: A method for characterizing molecular beams and beam reaction products. Chemical Physics Letters, 52, (1977). 
31. Houston, P. L. Two-dimensional imaging of state-selected photodissociation products detected by multiphoton ionization. J Chem Phys, 87, (1987).

32. Chandler, D. W. and Houston, P. L. Two-dimensional imaging of state-selected photodissociation products detected by multiphoton ionization. The Journal of Chemical Physics, 87, 1445-1447 (1987).

33. Parker, D. H. Velocity map imaging of ions and electrons using electrostatic lenses: Application in photoelectron and photofragment ion imaging of molecular oxygen. Rev Sci Instrum, 68, (1997).

34. Gebhardt, C. R., Rakitzis, T. P., Samartzis, P. C., Ladopoulos, V. and Kitsopoulos, T. N. Slice imaging: A new approach to ion imaging and velocity mapping. Rev Sci Instrum, 72, 3848-3853 (2001).

35. Li, W., Huang, C., Patel, M., Wilson, D. and Suits, A. State-resolved reactive scattering by slice imaging: A new view of the $\mathrm{Cl}+\mathrm{C}_{2} \mathrm{H}_{6}$ reaction. J Chem Phys, 124, 11102 (2006).

36. Proch, D. T., D. A high-intensity multi-purpose piezoelectric pulsed molecular beam source. Rev Sci Instrum, 60, (1989).

37. Abeysekera, C., Joalland, B., Shi, Y., Kamasah, A., Oldham, J. M. and Suits, A. G. Note: A short-pulse high-intensity molecular beam valve based on a piezoelectric stack actuator. Rev Sci Instrum, 85, 116107 (2014).

38. Lou, F. Phd thesis. University of Minnesota, (1995).

39. Zastrow, A. V., Onvlee, J., Parker, D. H. and van de Meerakker, S. Y. Analysis of velocity-mapped ion images from high-resolution crossed-beam scattering experiments: A tutorial review. EPJ Tech Instrum, 2, 11 (2015). 
40. Bontuyan, L. S., Suits, A. G., Houston, P. L. and Whitaker, B. J. State-resolved differential cross-sections for crossed-beam ar-no inelastic-scattering by direct ion imaging. J. Phys. Chem., 97, 6342-6350 (1993).

41. Suits, A. G., Bontuyan, L. S., Houston, P. L. and Whitaker, B. J. Differential cross-sections for state-selected products by direct imaging - Ar+NO. J. Chem. Phys., 96, 8618-8620 (1992).

42. Kohguchi, H., Suzuki, T. and Alexander, M. H. Fully state-resolved differential cross sections for the inelastic scattering of the open-shell no molecule by ar. Science, 294, 832-4 (2001).

43. Elioff, M. S. and Chandler, D. W. State-to-state differential cross sections for spin-multiplet-changing collisions of NO $\left(X^{2} \Pi\right)$ with argon. The Journal of Chemical Physics, 117, 6455-6462 (2002).

44. Eyles, C. J., Brouard, M., Chadwick, H., Hornung, B., Nichols, B., Yang, C. H., Klos, J., Aoiz, F. J., Gijsbertsen, A., Wiskerke, A. E. and Stolte, S. Fully lambda-doublet resolved state-to-state differential cross-sections for the inelastic scattering of no(x) with ar. Phys Chem Chem Phys, 14, 5403-19 (2012).

45. Gijsbertsen, A., de Lange M,J,L., Wiskerke, A,E., Linnartz, H., Drabbles, M., Klos, J., Stolte, S. Sign of the state-to-state asymmetry of rotationally inelastic atommolecule collisions. Chem. Phys, 301, 293-308 (2004).

46. Gijsbertsen, A., Linnartz H., Taatjes, C, A., Stolte, S. Quantum interfernce as the source of steric asymmetry and parity propensity rukes in no-rare gas inelastic scattering. J. AM. CHEM. SOC., 128, 8777 - 8789 (2006). 
47. Gijsbertsen, A., Linnartz, H., Rus, G., Wiskerke, A. E., Stolte, S., Chandler, D. W. and Klos, J. Differential cross sections for collisions of hexapole state-selected no with he. J Chem Phys, 123, 224305 (2005).

48. Alexander, M. H. and Stolte, S. Investigation of steric effects in inelastic collisions of NO ( $\left.X^{2} \Pi\right)$ with ar. J. Chem. Phys., 112, 8017-8026 (2000).

49. Eyles, C. J., Brouard, M., Yang, C. H., Klos, J., Aoiz, F. J., Gijsbertsen, A., Wiskerke, A. E. and Stolte, S. Interference structures in the differential cross-sections for inelastic scattering of no by ar. Nat Chem, 3, 597-602 (2011).

50. Lorenz, K. T., Chandler, D. W., Barr, J. W., Chen, W. W., Barnes, G. L. and Cline, J. I. Direct measurement of the preferred sense of no rotation after collision with argon. Science, 293, 2063-2066 (2001).

51. Onvlee, J., Vogels, S. N., Avoird, A. v. d., Groenenboom, G. C. and Meerakker, S. Y. T. v. d. Resolving rainbows with superimposed diffraction oscillations in no + rare gas scattering: Experiment and theory. New Journal of Physics, 17, 055019 (2015).

52. von Zastrow, A., Onvlee, J., Vogels, S. N., Groenenboom, G. C., van der Avoird, A. and van de Meerakker, S. Y. T. State-resolved diffraction oscillations imaged for inelastic collisions of no radicals with He, Ne and Ar. Nat. Chem., 6, 216-221 (2014). 53. James, P. L., Sims, I. R., Smith, I. W. M., Alexander, M. H. and Yang, M. A combined experimental and theoretical study of rotational energy transfer in collisions between $\mathrm{NO}\left(X^{2} \Pi, \mathrm{v}=3, \mathrm{j}\right)$ and $\mathrm{He}, \mathrm{Ar}$ and $\mathrm{N}_{2}$ at temperatures down to $7 \mathrm{k}$. The Journal of Chemical Physics, 109, 3882-3897 (1998). 
54. Kosanetzky, J., List, U., Urban, W., Vormann, H. and Fink, E. H. Vibrational relaxation of $\mathrm{NO}\left(X^{2} \Pi, v=1\right)$ studied by an ir-uv-double-resonance technique. Chem. Phys, 50, 361-371 (1980).

55. Sudbo, A. S. and Loy, M. M. T. State-to-state rotational and electronic collisional relaxation study of nitric oxide. Chem. Phys. Lett., 82, 135-137 (1981).

56. Dixit, A. A., Pisano, P. J. and Houston, P. L. Differential cross section for rotationally inelastic scattering of vibrationally excited no(v=5) from ar. J. Phys. Chem A., $105,11165-11170(2001)$.

57. Joalland, B., Van Camp, R., Shi, Y., Patel, N. and Suits, A. G. Crossed-beam slice imaging of cl reaction dynamics with butene isomers. J. Phys. Chem. A, 117, 7589-7594 (2013).

58. Cudry, F. O., J. Suits, A.G. Strong-field ionization of flash pyrolysis reaction products. J Phys Chem A, 119, (2015).

59. Kohn, D. W., Clauberg, H. and Chen, P. Flash pyrolysis nozzle for generation of radicals in a supersonic jet expansion. Rev. Sci. Instrum., 63, 4003 (1992).

60. Scharfenberg, L., Gubbels, K. B., Kirste, M., Groenenboom, G. C., van der Avoird, A., Meijer, G. and van de Meerakker, S. Y. T. Scattering of stark-decelerated oh radicals with rare-gas atoms. The European Physical Journal D, 65, 189-198 (2011).

61. Alexander, M. H. A new, fully ab initio investigation of the NO ( $\left.X^{2} \Pi\right)$ Ar system. I. Potential energy surfaces and inelastic scattering. J. Chem. Phys., 111, 7426-7434 (1999).

62. Booth, J. P., Bragg, S. L. and Hancock, G. Alignment effects in the multiple photon ionisation of nitric oxide. Chem. Phys. Lett., 113, 509-514 (1985). 
63. Jacobs, D. C., Madix, R. J. and Zare, R. N. Reduction of 1+1 resonance enhanced MPI spectra to population distributions: Application to the NO $\mathrm{A}^{2} \Sigma^{+}-X^{2} \Pi$ system. J. Chem. Phys., 85, 5469-5479 (1986).

64. Coudert, L. H., Dana, V., Mandin, J. Y., Morillonchapey, M. and Farrenq, R. The spectrum of nitric-oxide between 1700 and $2100 \mathrm{~cm}(-1)$. J. Mol. Spectrosc., 172, 435-448 (1995).

65. Paul, P. H. Calculation of transition frequencies and rotational line strengths in the $\gamma$-bands of nitric oxide. J. Quant. Spectrosc. Radiat. Transfer, 57, $581-589$ (1997). 66. Varberg, T. D., Stroh, F. and Evenson, K. M. Far-infrared rotational and finestructure transition frequencies and molecular constants of $14 \mathrm{NO}$ and $15 \mathrm{NO}$ in the $X^{2} \Pi$ $(v=0)$ state. J. Mol. Spectrosc., 196, 5-13 (1999).

67. PGOPHER. A program for simulating rotational, vibrational and electronic spectra. J. Quant. Spectrosc. Radiat. Transfer, 186, 221-242 (2016).

68. Bolton, O., Lee, K., Kim, H. J., Lin, K. Y. and Kim, J. Activating efficient phosphorescence from purely organic materials by crystal design. Nat Chem, 3, 205-210 (2011).

69. Goushi, K., Yoshida, K., Sato, K. and Adachi, C. Organic light-emitting diodes employing efficient reverse intersystem crossing for triplet-to-singlet state conversion. Nat Photonics, 6, 253-258 (2012).

70. Zhao, J. Z., Wu, W. H., Sun, J. F. and Guo, S. Triplet photosensitizers: From molecular design to applications. Chem. Soc. Rev., 42, 5323-5351 (2013).

71. Kamkaew, A., Lim, S. H., Lee, H. B., Kiew, L. V., Chung, L. Y. and Burgess, K. Bodipy dyes in photodynamic therapy. Chem. Soc. Rev., 42, 77-88 (2013). 
72. Alagia, M., Balucani, N., Cartechini, L., Casavecchia, P., van Beek, M., Volpi, G. G., Bonnet, L. and Rayez, J. C. Crossed beam studies of the $\mathrm{O}\left({ }^{3} \mathrm{p},{ }^{1} \mathrm{~d}\right)+\mathrm{CH}_{3} \mathrm{I}$ reactions: Direct evidence of intersystem crossing. Faraday. Discuss., 113, 133-150 (1999).

73. Koziar, J. C. and Cowan, D. O. Photochemical heavy-atom effects. Accounts Chem Res, 11, 334-341 (1978).

74. Casavecchia, P., Leonori, F. and Balucani, N. Reaction dynamics of oxygen atoms with unsaturated hydrocarbons from crossed molecular beam studies: Primary products, branching ratios and role of intersystem crossing. Int. Rev. Phys. Chem., 34, 161-204 (2015).

75. Fu, B. N., Han, Y. C., Bowman, J. M., Angelucci, L., Balucani, N., Leonori, F. and Casavecchia, P. Intersystem crossing and dynamics in $\mathrm{O}\left({ }^{3} \mathrm{p}\right)+\mathrm{C}_{2} \mathrm{H}_{4}$ multichannel reaction: Experiment validates theory. P Natl Acad Sci USA, 109, 9733-9738 (2012). 76. Leonori, F., Balucani, N., Nevrly, V., Bergeat, A., Falcinelli, S., Vanuzzo, G., Casavecchia, P. and Cavallotti, C. Experimental and theoretical studies on the dynamics of the $\mathrm{O}\left({ }^{3} \mathrm{p}\right)+$ propene reaction: Primary products, branching ratios, and role of intersystem crossing. J Phys Chem C, 119, 14632-14652 (2015).

77. Leonori, F., Occhiogrosso, A., Balucani, N., Bucci, A., Petrucci, R. and Casavecchia, P. Crossed molecular beam dynamics studies of the $\mathrm{O}\left({ }^{3} \mathrm{p}\right)+$ allene reaction: Primary products, branching ratios, and dominant role of intersystem crossing. J Phys Chem Lett, 3, 75-80 (2012).

78. Schmoltner, A. M., Chu, P. M., Brudzynski, R. J. and Lee, Y. T. Crossed molecular beam study of the reaction $\mathrm{O}\left({ }^{3} \mathrm{p}\right)+\mathrm{C}_{2} \mathrm{H}_{4}$. The Journal of Chemical Physics, 91, 6926-6936 (1989). 
79. Schmoltner, A. M., Huang, S. Y., Brudzynski, R. J., Chu, P. M. and Lee, Y. T. Crossed molecular-beam study of the reaction $\mathrm{O}\left({ }^{3} \mathrm{p}\right)+$ allene. Journal of Chemical Physics, 99, 1644-1653 (1993).

80. Kohse-Hoinghaus, K., Osswald, P., Cool, T. A., Kasper, T., Hansen, N., Qi, F., Westbrook, C. K. and Westmoreland, P. R. Biofuel combustion chemistry: From ethanol to biodiesel. Angew. Chem. Int. Ed. Engl., 49, 3572-3597 (2010).

81. Lucassen, A., Zhang, K. W., Warkentin, J., Moshammer, K., Glarborg, P., Marshall, P. and Kohse-Hoinghaus, K. Fuel-nitrogen conversion in the combustion of small amines using dimethylamine and ethylamine as biomass-related model fuels. Combust Flame, 159, 2254-2279 (2012).

82. Miller, J. A. and Bowman, C. T. Mechanism and modeling of nitrogen chemistry in combustion. Progress in Energy and Combustion Science, 15, 287-338 (1989).

83. Li, S. J., Davidson, D. F. and Hanson, R. K. Shock tube study of dimethylamine oxidation. Int. J. Chem. Kinet., 47, 19-26 (2015).

84. Lucassen, A., Labbe, N., Westmoreland, P. R. and Kohse-Hoinghaus, K. Combustion chemistry and fuel-nitrogen conversion in a laminar premixed flame of morpholine as a model biofuel. Combust Flame, 158, 1647-1666 (2011).

85. Joalland, B., Shi, Y. Y., Estillore, A. D., Kamasah, A., Mebel, A. M. and Suits, A. G. Dynamics of chlorine atom reactions with hydrocarbons: Insights from imaging the radical product in crossed beams. Journal of Physical Chemistry A, 118, 9281-9295 (2014).

86. Li, W., Chambreau, S. D., Lahankar, S. A. and Suits, A. G. Megapixel ion imaging with standard video. Rev. Sci. Instrum., 76, (2005). 
87. Montgomery, J. A., Frisch, M. J., Ochterski, J. W. and Petersson, G. A. A complete basis set model chemistry. Vii. Use of the minimum population localization method. J. Chem. Phys., 112, 6532-6542 (2000).

88. Montgomery, J. A., Frisch, M. J., Ochterski, J. W. and Petersson, G. A. A complete basis set model chemistry. Vii. Use of the minimum population localization method. Journal of Chemical Physics, 112, 6532 (2000).

89. Atkinson, R. and Pitts, J. N. Kinetics of the reactions of $\mathrm{O}\left({ }^{3} \mathrm{p}\right)$ atoms with the amines $\mathrm{CH}_{3} \mathrm{NH}_{2}, \mathrm{C}_{2} \mathrm{H}_{5} \mathrm{NH}_{2},\left(\mathrm{CH}_{3}\right)_{2} \mathrm{NH}$, and $\left(\mathrm{CH}_{3}\right)_{3} \mathrm{~N}$ over the temperature range 298$440{ }^{\circ} \mathrm{k}$. Journal of Chemical Physics, 68, 911-915 (1978).

90. Slagle, I. R., Dudich, J. F. and Gutman, D. Identification of reactive routes in the reactions of oxygen atoms with methylamine, dimethylamine, trimethylamine, ethylamine, diethylamine, and triethylamine. The Journal of Physical Chemistry, 83, 3065-3070 (1979).

91. Balucani, N., Leonori, F., Casavecchia, P., Fu, B. N. and Bowman, J. M. Crossed molecular beams and quasiclassical trajectory surface hopping studies of the multichannel nonadiabatic $\mathrm{O}\left({ }^{3} \mathrm{P}\right)+$ ethylene reaction at high collision energy. Journal of Physical Chemistry A, 119, 12498-12511 (2015).

92. Herron, J. T. and Huie, R., H. Rate of reaction of oxygen. $\mathrm{C}_{2}$ to $\mathrm{C}_{8}$ alkanes. Journal of Physical Chemistry, (1969).

93. Warnatz, J. and Gardiner, W., C. Combustion chemistry. Springer, (1984).

94. Rowland, F. S. and Molina, M. J. Chlorofluoromethanes in the environment. Reviews of Geophysics, (1975). 
95. Luntz, C. and Andersen, J. The chemical dynamics of the reactions of o(3p) with saturated hydrocarbons. Ii. Theoretical model. J Chem Phys, 72, (1980).

96. Kleinermanns, K. and Luntz, C. The chemical dynamics of hydrogen atom abstraction from unsaturated hydrocarbons by $\mathrm{O}\left({ }^{3} \mathrm{P}\right)$. J Chem Phys, 117, (1982).

97. Liu, X. H., Gross, R. L., Hall, G. E. and Suits, A. Imaging $\mathrm{O}\left({ }^{3} \mathrm{P}\right)+$ alkane alkane reactions in crossed molecular beams: Vertical versus adiabatic $\mathrm{h}$ abstraction dynamics. Journal of Chemical Physics, 117, 710-716 (2002).

98. Gross, R. L., Liu, X. H. and Suits, A. $\mathrm{O}\left({ }^{3} \mathrm{P}\right)$ versus o(1d) reaction dynamics with n-pentane: A crossed-beam imaging study. Chemical Physics Letters, 376, (2003).

99. Sibener, S. J., Buss, R. J., Casavecchia, P. and Lee, Y. T. A crossed molecular beams investigation of the reactions $\mathrm{O}\left({ }^{3} \mathrm{P}\right)+\mathrm{C}_{6} \mathrm{H}_{6}, \mathrm{C}_{6} \mathrm{D}_{6}$. Journal of Chemical Physics, 72 , (1980).

100. Harper, M. R., Van Geem, K. M., Pyl, S. P., Marin, G. B. and Green, W. H. Comprehensive reaction mechanism for n-butanol pyrolysis and combustion. Combust Flame, 158, 16-41 (2011).

101. Lowry, S. O. and Devoto, R. S. Exhaust emissions from a single-cylinder engine fueled with gasoline, methanol, and ethanol. Journal Combustion of Science and Technology, 12, (1976).

102. Moss, J. T., Berkowitz, A. M., Oehlschlaeger, M. A., Biet, J., Warth, V., Glaude, P. A. and Battin-Leclerc, F. An experimental and kinetic modeling study of the oxidation of the four isomers of butanol. Journal of Physical Chemistry A, 112, 10843-10855 (2008). 
103. Sarathy, S. M., Thomson, M. J., Togbe, C., Dagaut, P., Halter, F. and MounaimRousselle, C. An experimental and kinetic modeling study of n-butanol combustion. Combust Flame, 156, 852-864 (2009).

104. Kato, A. and Cvetanović, R. J. Reaction of oxygen atoms with ethanol. . Can. J. Chem., 45, (1967).

105. Kato, A. and Cvetanović, R. J. Reactions of oxygen atoms with 2-propanol and methanol. Can. J. Chem., (1968).

106. Lefevre, H., F, Meagher, J. F. and Timmons, R. B. The kinetics of the reactions of o(3p) atoms with dimethyl ether and methanol. Int. J. Chem. Kinet., 4, 103-116 (1972).

107. Owens, C. M. and Roscoe, J. M. The reactions of atomic oxygen with methanol and ethanol. Can. J. Chem., 54, 984-989 (1967).

108. Luo, N., Kombo, D. C. and Osman, R. Theoretical studies of hydrogen abstraction from 2-propanol by oh radical. Journal of Physical Chemistry A, 101, 926-936 (1997). 109. Hess, W., P and Tully, F., P. Hydrogen atom abstraction from methanol by oh. Journal of Physical Chemistry, 93, (1989).

110. Taatjes, C. A., Christensen, L. K., Hurley, M. D. and Wallington, T. J. Absolute and site-specific abstraction rate coefficients for reactions of cl with $\mathrm{CH}_{3} \mathrm{CH}_{2} \mathrm{OH}$, $\mathrm{CH}_{3} \mathrm{CD}_{2} \mathrm{OH}$, and $\mathrm{CD}_{3} \mathrm{CH}_{2} \mathrm{OH}$ between 295 and $600 \mathrm{k}$. Journal of Physical Chemistry A, 103, 9805-9814 (1999).

111. Ahmed, M., Peterka, D. S. and Suits, A. G. H abstraction dynamics by crossedbeam velocity map imaging: $\mathrm{Cl}+\mathrm{CH}_{3} \mathrm{OH}->\mathrm{CH}_{2} \mathrm{OH}+\mathrm{HCl}$. Chemical Physics Letters, 317, 264-268 (2000). 
112. Ahmed, M., Peterka, D. S. and Suits, A. G. Imaging h abstraction dynamics in crossed molecular beams: $\mathrm{Cl}+\mathrm{ROH}$ reactions. Phys. Chem. Chem. Phys., 2, 861-868 (2000).

113. Estillore, A. D., Visger-Kiefer, L. M. and Suits, A. G. Reaction dynamics of cl plus butanol isomers by crossed-beam sliced ion imaging. Faraday. Discuss., 157, 181$191(2012)$.

114. Bechtel, H. A., Camden, J. P. and Zare, R. N. State-to-state dynamics of the $\mathrm{Cl}+\mathrm{CH}_{3} \mathrm{OH}->\mathrm{HCl}+\mathrm{CH}_{2} \mathrm{O}$ reaction. Journal of Chemical Physics, 120, 4231-4239 (2004).

115. Murray, C., Orr-Ewing, A. J., Toomes, R. L. and Kitsopoulos, T. N. Imaging the quantum-state specific differential cross sections of $\mathrm{HCl}$ formed from reactions of chlorine atoms with methanol and dimethyl ether. Journal of Chemical Physics, 120, 2230-2237 (2004).

116. Wiesenfeld, J. and Goldstein, N. Dynamics of $O\left({ }^{1} \mathrm{D}\right)$ reactions with bifunctional substrates: Alcohols and thiols. J Chem Phys, 78, (1983).

117. Joalland, B., Van Camp, R. D., Shi, Y., Patel, N. and Suits, A. G. Crossed-beam slice imaging of cl reaction dynamics with butene isomers. Journal of Physical Chemistry A, (2013).

118. Felder, P., Effenhauser, Hass, B. M. and Huber, J. R. Photodissociation of sulfur dioxide at $193 \mathrm{~nm}$. Chemical Physics Letters, 148, (1988).

119. Kawasaki, M. and Sato, K. Photodissociation of molecular beams of $\mathrm{SO}_{2}$ at 193 nm. Chemical Physics Letters, 139, (1987).

120. Ayub, A. L. and Roscoe, J. M. The reaction of ground state atomic oxygen with 1-propanol and 2-propanol has been studied with kinetic methods and the reaction rates 
of the H-abstraction from C-H group were measured. Can. J. Chem., 57, 1269-1273 (1979).

121. Lee, S. H., Dong, F. and Liu, K. P. Reaction dynamics of F+HD -> HF+D at low energies: Resonant tunneling mechanism. Journal of Chemical Physics, 116, 7839-7848 (2002). 


\section{VITA}

Alexander Kamasah was born and grew up in Accra Ghana. He obtained his undergraduate degree from the university of Cape-Coast and relocated to the United States in the year 2010 for his M.Sc. degree (Analytical chemistry) at East Tennessee State University in Johnson City Tennessee. He then gained admission to Wayne State University in Detroit Michigan for his Ph.D. in physical chemistry, where He joined the Suits lab and studied crossed beam reaction dynamics. During the course of his Ph.D. program his supervisor relocated his lab to University of Missouri and he followed him to the university of Missouri. He completed his Ph.D. program in July 2018. 